\title{
Review of 2D superconductivity: the ultimate case of epitaxial monolayers
}

\author{
Christophe Brun $^{1},{ }^{*}$ Tristan Cren $^{1}$, and Dimitri Roditchev ${ }^{1,2}$ \\ ${ }^{1}$ Institut des Nanosciences de Paris, Sorbonne université, \\ UPMC univ Paris 6 and CNRS-UMR 7588, 4 place Jussieu, 75252 Paris, France and \\ ${ }^{2}$ Laboratoire de physique et d'études des matériaux, \\ LPEM-UMR8213/CNRS-ESPCI ParisTech-UPMC, 10 rue Vauquelin-75005 Paris, France
}

(Dated: September 30, 2016)

\begin{abstract}
The purpose of this review is to focus from an experimental point-of-view on the new physical properties of some of the thinnest superconducting films that can be fabricated and studied in situ nowadays with state-of-the-art methods. An important characteristic of the films we address is that the underlying electronic system forms a two-dimensional electron gas (2DEG). Up to now there are only few of these systems. Such true 2D superconductors can be divided into two classes: surface-confined or interface-confined films. Because the second types of films are burried below the surface, they are not accessible to purely surface-sensitive techniques like angular-resolved photoemission spectroscopy (ARPES) or scanning tunneling spectroscopy (STS). As a consequence the bandstructure characteristics of the 2DEG cannot be probed nor the local superconducting properties. On the other hand, in situ prepared surface-confined films are nowadays accessible not only to ARPES and STS but also to electrical transport measurements. As a consequence surface-confined systems represent at present the best archetypes on which can be summarized the new properties emerging in ultimately thin superconducting films hosting a two-dimensional electron gas, probed by both macroscopic and microscopic measurement techniques. The model system we will widely refer to consists of a single atomic plane of a conventional superconductor, like for example lead $(\mathrm{Pb})$, grown on top of a semiconducting substrate, like $\mathrm{Si}(111)$. In the introductory part (I) we first introduce the topic and give historical insights into this field. Then in the section II, we introduce useful concepts worked out in studies of so-called "granular" and "homogeneous" superconducting thin films that will be necessary to understand the role of non-magnetic disorder on 2DEG superconductors. In this section, we also briefly review the superconducting properties of crystalline $\mathrm{Pb} / \mathrm{Si}(111)$ ultrathin films grown under ultrahigh vacuum (UHV) conditions in order to illustrate their specific properties related to quantum-size effects. In the next section (III) we review the growth methods and structural properties of the presented 2DEG surface-confined superconductors. In section (IV), we review the electronic structure and Fermi surface properties as measured by macroscopic ARPES and confront them to ab initio DFT calculations based on the characterized atomic structures of the monolayers. The following section (V) reviews the macroscopic properties inferred from in situ electrical transport measurements methods, including attempts to study the Berezinsky-Kosterlitz-Thouless 2D regime. In the last section (VI), we summarize the emerging local spectroscopic properties measured by STS. These latter demonstrate variations of the local superconducting properties at a scale much shorter than the superconducting coherence length due to a combined effect of non-magnetic disorder and two-dimensionality. Further peculiar local spectroscopic effects are presented giving evidence for the presence of a mixed singlet-triplet superconducting order parameter induced by the presence of a strong Rashba spin-orbit coupling term at the surface. These local signatures will be discussed along with ARPES and transport measurements in parallel high magnetic field on closely related systems. Finally, we present in anisotropic $\mathrm{Pb}$ and In monolayers the peculiar role played by atomic steps on vortex properties, leading to the observation by STS of mixed Abrikosov-Josephson vortices in agreement with in situ macroscopic transport measurements. From the overview of all recent experimental and theoretical results it appears that these surface $2 \mathrm{D}$ superconductors, such as one monolayer of $\mathrm{Pb}$ on $\mathrm{Si}(111)$, are ideal templates to engineer and realize topological superconductivity.
\end{abstract}

PACS numbers: $74.45 .+\mathrm{c}, 74.55 .+\mathrm{v}, 74.78 .-\mathrm{w}, 74.78 . \mathrm{Na}$

\section{Contents}

\section{Introduction}

II. Basic properties of ultrathin disordered s-wave superconductors

A. Granular superconducting films

B. Disordered homogeneous superconducting films

C. Crystalline ultrathin superconducting films 7
III. Monolayer films growth and structural properties

\section{Monolayers electronic properties from ARPES measurements confronted to $a b$ initio DFT calculations}

\section{Superconducting properties from in situ} magneto-transport measurements 
perpendicular magnetic field

B. Critical current and role of atomic steps

C. magneto-transport measurements in parallel magnetic field : hint for a spin-triplet component of the order parameter

D. Berezinski-Kosterlitz-Thouless transition in monolayer superconducting films

\section{Local superconducting properties from scanning tunneling spectroscopy measurements}

A. New inhomogeneous superconducting properties in monolayer superconductors induced by $2 \mathrm{D}$ electronic dimensionality : short-range spatial variations of the height of coherence peaks

B. In-gap states induced by non-magnetic disorder in monolayer superconductors : hint for a mixed singlet-triplet superconducting order parameter induced by strong Rashba spin-orbit coupling

C. Vortex properties and role of step edges in monolayer superconducting films

1. zero magnetic field properties at step edges

2. magnetic field properties at step edges

\section{Conclusion}

VIII. Acronyms

\section{Acknowledgments}

\section{References}

\section{INTRODUCTION}

Early in 1964 Ginzburg and Kirzhnits have conjectured the possible existence of superconductivity being confined at the very surface of a solid ${ }^{1}$. They had in mind a truly two-dimensional superconductor in the sense that the electron gas itself would be localized on the surface of the material and would form a two-dimensional electronic system. As experimental realizations Ginzburg and Kirzhnits imagined two systems. In the first one the bulk material would be a dielectric hosting Tamm surface states which filling could be induced and controlled by charge transfer. An attractive electron-phonon coupling should then develop in the surface layer to induce superconductivity, possibly involving surface phonons. As a second system they imagined a bulk metal also hosting Tamm surface states. In order to enable revealing intrinsic surface superconductivity, the attractive coupling between electrons and phonons should be larger for surface electrons than for bulk electrons ${ }^{2}$. Very interestingly, due to a softening of phonon modes at the surface, Ginzburg envisioned the possibility to obtain high-Tc surface superconductors in both of these systems. Surface engineering with suitable light-element-molecules was also mentioned as a way to enhance the critical temperature, following the ideas of Little $^{3}$.

26 Historically, the study of clean surface or interface systems was long hindered by technical limitations in the cleanliness of the preparation processes. First of all, the search for the thinnest superconductors began with the study of ultrathin superconducting films deposited on an insulating or semiconducting substrate, using the physical vapor deposition method through resistive Joule heating ${ }^{4}$. These films usually consisted of nanocrystallites or crystalline grains, structurally and electrically coupled togoether. Depending on the electrical coupling between neighboring grains, the obtained superconducting films can be divided into two classes of systems, so-called granular and homogeneous systems.

As we explain below in section II, the development of this field brought forward important new concepts and clarifications about the role of non-magnetic disorder in conventional ultrathin superconductors. Nevertheless, the preparation conditions did not enable fabricating true 2D-electron-gas based superconducting systems. This does not mean that 2D-superconductivity could not be reached : on the contrary, as soon as the film thickness $d$ is smaller than the superconducting coherence length in the direction perpendicular to the film's surface, 2D-superconductivity is realized. Typically the thinnest granular or homogeneous films studied there are about a few nanometers thick and do not superconduct above a critical disorder. This threshold for the destruction of superconductivity occurs when $k_{F} l_{e}$ decreases toward unity $\left(k_{F}\right.$ being the Fermi wave vector and $l_{e}$ the elastic 
electronic mean-free-path). In terms of the macroscopic sample resistivity, superconductivity disappears when the square resistance of the film becomes comparable to the quantum of resistance for electron pairs $\left(R_{\text {square }} \simeq h / 4 e^{2}=6.45 k \Omega\right)$. $R_{\text {square }}$ corresponds to the resistance of a square film, thus $R_{\text {square }}=\rho L / d w$ where $\rho$ is the electrical resistivity $d, w, L$ being respectively the sample thickness, width, and length. For a square film $l=w$ and thus $R_{\text {square }}=\rho / d$.

The advent of molecular beam epitaxy (MBE) and surface physics methods, with the use of ultrahigh vacuum (UHV) techniques and highly purified source materials, enabled bridging the gap. Favorable growth conditions were obtained in order to create clean surface and interface systems. Up to now, the widely studied semiconducting III-V of II-VI heterostructures, like for instance the very well-known GaAs/AlGaAs 2D electron gas system (2DEG), which allowed the discovery of the integer and fractionnal quantum Hall effect, did not reveal any superconducting behavior. The first system that appeared to be close to a true 2DEG system and showed a superconducting transition below $1 \mathrm{~K}$ is $\mathrm{LaAlO}_{3} / \mathrm{SrTiO}_{3}{ }^{5,6}$. This oxyde heterostructure is built out of two insulating parts. At the interface, a charge transfer occurs to release strong electric polarization effects in the upper $\mathrm{LaAlO}_{3}$ layer. As a result an interface electron gas is formed about $10 \mathrm{~nm}$ below the surface, which electro-chemical potential can be tuned by electrostatic gating. This gating allows the system to be tuned from a superconducting state with a maximum critical temperature to an insulator when the 2DEG is fully depleted. The microscopic pairing mechanism in these systems is still debated and under study. A recent overview of superconductivity in oxydes interfaces can be found by Gariglio et al. ${ }^{7}$

As these types of interface films are burried below the surface, they are not accessible to purely surfacesensitive techniques like angular-resolved photoemission spectroscopy (ARPES) or scanning tunneling spectroscopy (STS). As a consequence the bandstructure characteristics of the 2DEG cannot be probed nor the local superconducting properties. On the other hand, in situ prepared surface-confined films grown by MBE are nowadays accessible not only to in situ ARPES and STS but also to in situ magneto-electrical transport measurements and structural characterization tools like electronic or optical surface diffraction. As a consequence surface-confined systems represent at present the best archetypes on which can be summarized the new properties emerging in ultimately thin superconducting films hosting a two-dimensional electron gas, because they can be probed by both in situ macroscopic and microscopic measurement techniques. The model system we will widely refer to in this review consists of a single atomic plane of a conventional superconductor, like for example lead $(\mathrm{Pb})$, grown on top of a semiconducting substrate, like $\mathrm{Si}(111)^{8}$. By conventional supercon- ductivity we mean that the pairing interaction is of electron-phonon type.

We decided also in this paper not to focus on recent advances in studying few layers, bilayers or monolayers of dichalcogenides materials. In these systems conventional superconductivity is most probably at play. This includes for example the study of ultrathin $\mathrm{MoS}_{2}$ or $2 \mathrm{H}-\mathrm{NbSe}_{2}$ films, using various surface encapsulation techniques to protect the layers and/or gating techniques to reach the $2 \mathrm{D}$ electronic regime of superconductivity ${ }^{9-14}$. We believe that although very interesting results were obtained in this field recently, these measurements consist in almost exclusively magneto-electrical transport experiments. This is way too lacunar yet to furnish an overview of their precise superconducting properties from a multi-techniques approach. For similar reasons we will not address recent results obtained by inducing superconductivity at the surface of $\mathrm{SrTiO}_{3}{ }^{15}$ or layered $\mathrm{ZrNCl}^{16}$ using an electric-double-layer gating in an organic electrolyte, or at the surface of graphene using proximity effects ${ }^{17,18}$.

We also mention that it is beyond the scope of this review paper to cover the recent advances obtained in the field of unconventional superconductivity, where the pairing mechanism is not mediated by an electronphonon interaction. In particular very promising results were obtained by fabricating in UHV ultrathin cuprates films ${ }^{19}$. In the field of iron-based superconductivity, trumendous effects of the control of the interface and substrate termination were demonstrated for FeSe films using an $\mathrm{SrTiO}_{3}$ substrate ${ }^{20,21}$. A critical temperature above $100 \mathrm{~K}$ was reported for one unit cell of $\mathrm{FeSe} / \mathrm{SrTiO}_{3}$, which is one order of magnitude larger than the bulk FeSe critical temperature. There is thus hope in these fields both to better study and understand the unconventional superconducting properties and to engineer a higher critical temperature by playing suitably with the substrate.

We will adopt in our review the following plan. In the next section II, we will introduce useful concepts worked out in studies of so-called "granular" and "homogeneous" superconducting thin films that will be necessary to understand the role of non-magnetic disorder on 2DEG superconductors. In this section, we will also briefly review the superconducting properties of crystalline $\mathrm{Pb} / \mathrm{Si}(111)$ ultrathin films grown under ultrahigh vacuum (UHV) conditions in order to illustrate their specific properties related to quantum-size effects and thickness reduction. In the section (III) we will review the growth methods and structural properties of the presented 2DEG surface-confined superconductors. In section (IV), we will present the electronic structure and Fermi surface properties as measured by macroscopic ARPES and confront them to ab initio DFT calculations based on the characterized atomic 
structures of the monolayers. The following section (V) will summarize the macroscopic properties inferred from ex situ and in situ magneto-electrical transport measurements, including recent attempts to study the Berezinsky-Kosterlitz-Thouless 2D regime. In the last section (VI), we will summarize the emerging local spectroscopic properties measured by STS. These latter demonstrate new short-range spatial variations of the local superconducting properties due to a combined effect of non-magnetic disorder and two-dimensionality, and peculiar in-gap states giving evidence for the presence of a mixed singlet-triplet superconducting order parameter induced by the presence of a strong Rashba spin-orbit coupling term at the surface. These local signatures will be discussed along with ARPES and in situ transport measurements in parallel high-magnetic field on closely related systems. Finally, the peculiar role played by atomic steps in anisotropic monolayers on vortex properties, leading to the observation of mixed Abrikosov-Josephson vortices in agreement with in situ macroscopic transport measurements will be presented. In a last section, we will conclude and summarize the key-results obtained using both local and macroscopic measurement techniques. We will also figure out the remaining important issues to be clarified in monolayer superconductors. From the overview of all recent experimental and theoretical results it will appear that these surface 2D superconductors, such as one monolayer of $\mathrm{Pb}$ on $\mathrm{Si}(111)$, are ideal templates to engineer and realize topological superconductivity.

\section{BASIC PROPERTIES OF ULTRATHIN DISORDERED S-WAVE SUPERCONDUCTORS}

Real materials are not pure and contain impurities or structural defects which may deeply alter their electronic properties. It was shown by Abrikosov, Gorkov ${ }^{22}$ and Anderson $^{23}$ that the presence of non-magnetic disorder in s-wave superconductors does not alter their thermodynamic properties, upon the assumption of time-reversal symmetry. Later on, detailed experimental and theoretical investigations have addressed over decades the effect of disorder on the properties of superconductors, in particular through thickness reduction. As a result, several complicated situations may occur, depending on the interplay between structural and electronic properties of the material. Some of these aspects are reviewed briefly by Goldman and Markovic ${ }^{24}$ and thoroughly by Feigel'man et al. ${ }^{25}$ and by Gantmakher and Dolgopolov $^{26}$. In general a transition to a metallic or insulating state occurs beyond a critical disorder, which is achieved when the film's square resistance becomes comparable to $R_{\text {square }} \simeq h / 4 e^{2}$.
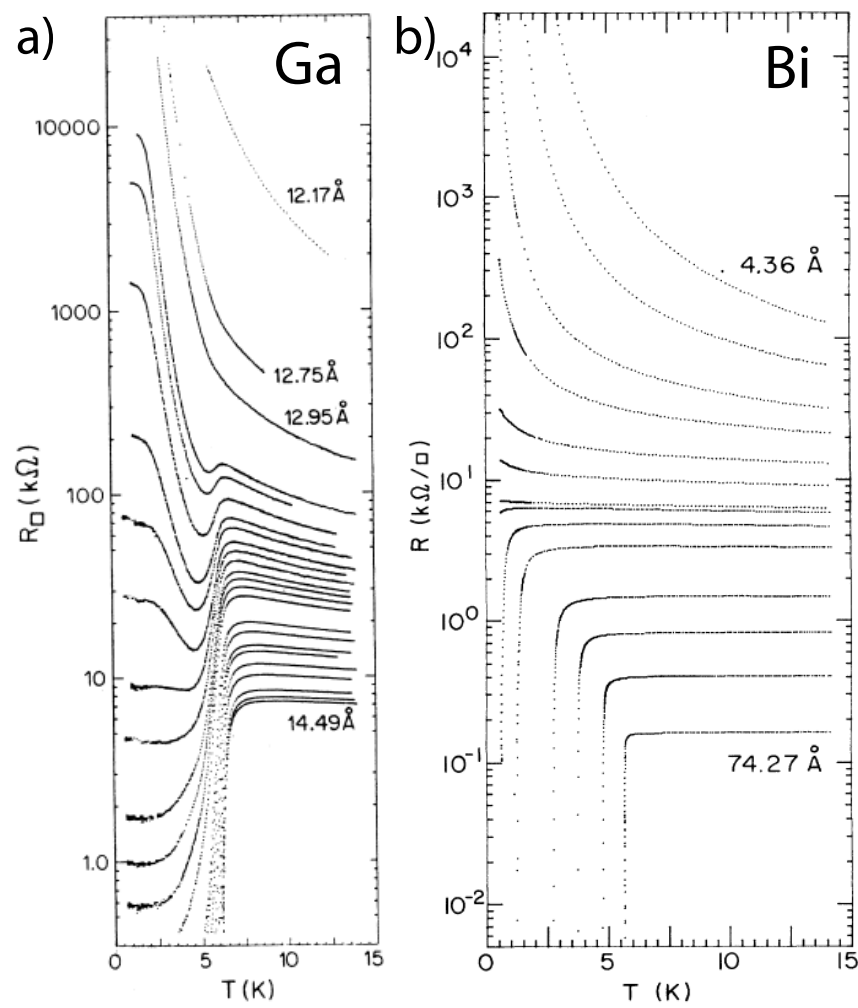

FIG. 1: Illustration of the different behaviors observed in so-called granular and homogeneous disordered superconducting ultrathin films. Evolution of the temperature dependence of the square resistance of Gallium and Bismuth ultrathin films. Each curve corresponds to a deposited thickness given in $\AA$. (a) Granular Ga films deposited by the quench-condensed method on a quartz substrate. Data reproduced from Jaeger et al. ${ }^{31}$. (b) Bi films grown by the same method but deposited on a Ge-coated layer. Data reproduced from Haviland et al. ${ }^{34}$. The thickness increments are respectively of $0.05-0.1 \AA$ for $\mathrm{Ga}$ and $5.4 \AA$ for Bi.

\section{A. Granular superconducting films}

Historically, so-called granular materials were synthetized first using the quench condensation method ${ }^{4}$. Usually these thin films were synthetized by evaporating a metallic source onto a cold dielectric substrate consisting of glass or quartz. These films consist of grains or nanocrystallites oxydized at their surface because they were prepared in a rather high vacuum pressure. Thus these grains are coupled together by low-transparency tunnel junctions: the electrons can not directly hop from one grain to the next but should tunnel to be transferred from one nanocrystal to the other. As a consequence the electrical coupling between neighboring grains is rather poor and Coulombic effects start to play an important role through the charging energy associated to each grain. The grain's charging energy is $E_{c}=e^{2} / 2 C$, where $e$ is the electronic charge and $C$ the grain's effective capacitance. It represents the Coulombic energy barrier necessary to inject (extract) one electron into a particular grain ${ }^{27}$. 
In granular materials, the charging energy $E_{c}$ compeats with another energy scale, the Josephson energy $E_{J}$, related to the establishment of superconductivity in the whole film ${ }^{28-31}$ by coupling the superconducting phases of neighboring grains. The Josephson energy is given by $E_{J}=\Phi_{0} I_{c}, \Phi_{0}=h / 2 e$ being the flux quantum and $I_{c}$ the critical current, i.e. the maximum supercurrent, of a Josephson junction. As the grains are electronically poorly coupled to each other, superconductivity develops in each grain labelled by the index $j$ with its own order parameter $\Delta_{j} \exp \left(i \Phi_{j}\right)$ (amplitude $\Delta_{j}$ and phase $\Phi_{j}$ ) and couples from one grain to the next by the Josephson effect.

The ratio between the Josephson and the charging energy characterizes how Cooper pairs can tunnel from one grain to the neighboring one and establish a long range phase coherence of the superconducting order $\left(E_{J} \gg E_{c}\right)$ or in the contrary be blocked on grains by charging effects $\left(E_{c} \gg E_{J}\right)$. The typical evolution of the critical temperature as a function of film thickness $d$ is seen on Fig. 1(a). The temperature onset, where the resistance starts to decrease from above, does not change with thickness. This indicates that the superconducting transition temperature remains roughly constant and over a thickness change of less than one monolayer the films go to an insulating state. To summarize the granular superconductors's properties: there is a competition between Coulomb blockade and Josephson coupling and the $T_{c}$ of the whole film is given by the elementary grain's $T_{c}$ which does not change much upon thickness reduction.

\section{B. Disordered homogeneous superconducting films}

With the progress in deposition techniques, so-called disordered homogeneous superconducting films could be grown ${ }^{24,25,32-34}$. As shown in Fig. 1(b), they are characterized by a gradual reduction of their critical temperature $T_{c}$ with decreasing thickness $d$. This behavior is at variance with the one seen in Fig. 1(a). In contrast to the synthesis process used forgranular films described above, a germanium few monolayer thick film is deposited first on top of the dielectric substrate. This provides dangling bonds at the surface making the growth of metal films much more smooth and homogeneous on the Gecoated substrate. These disordered homogeneous films consist of nanocrystals well-coupled electrically to each other: in contrast to granular films there are no weak links between the neighboring nanocrystal. This situation is in practice achieved in many ultrathin films: $\mathrm{Pb}$, In, Bi, Al, MoGe, nitrides like TiN or $\mathrm{NbN}^{24,25,32,33}$. In all these systems (about $2 \mathrm{~nm}$ thick for the thinnest) the superconducting properties are two-dimensional (2D), as the film thickness $d$ is smaller than the low-temperature superconducting coherence length $\xi_{0}$, but the underlying electronic wavefunctions are still three-dimensional (3D) as $\lambda_{F}$ remains significantly smaller than $d$.

The mechanisms driving the film from a super-

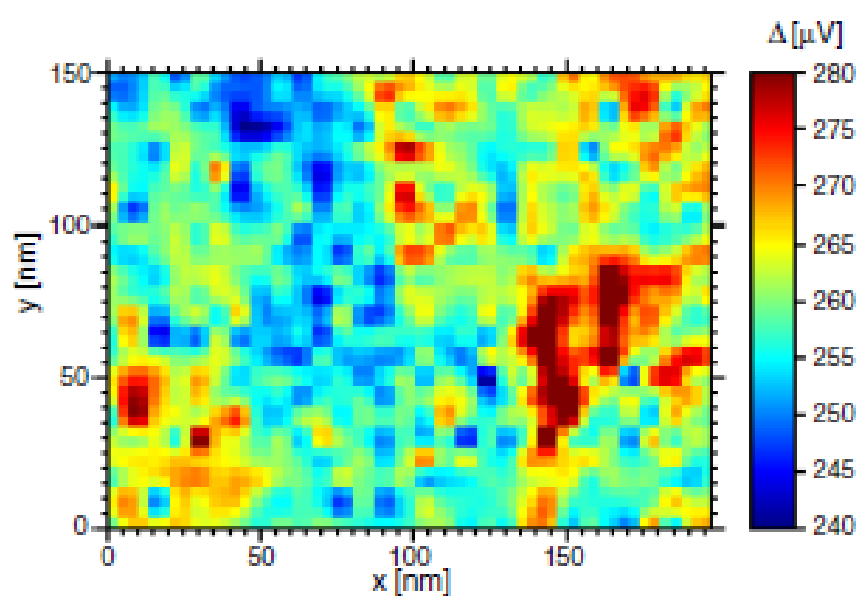

FIG. 2: Spatial gap variations observed in so-called homogeneous disordered superconducting ultrathin films. The color map represents the superconducting gap measured locally by STS in a $3.6 \mathrm{~nm}$ thick TiN film grown ex situ on $\mathrm{Si} / \mathrm{SiO}_{2}$ substrate. The superconducting inhomogeneities show up on a scale of few tens of nanometers. The film's temperature dependent electrical resistivity and typical STS spectrum are shown in Fig. 4 for film TiN1. Adapted from Sacépé et $a .^{39}$.

conducting to an insulating or metallic state are a complex combination of Coulombic effects and Anderson localization effects, both being induced by increasing disorder ${ }^{24,25}$.

Coulombic effects alone lead to the so-called Fermionic scenario $^{35}$. It is characterized by enhanced Coulomb repulsion between delocalized electrons because increased scattering due to high disorder provokes a reduced electronic screening. This reduced electronic screening was originally predicted by Altshuler and Aronov in disordered metallic systems. They showed that this effect results in a spectroscopic signature : there is a reduced electronic density of states (DOS) at the Fermi level. This effect is now referred to as the Altshuler-Aronov anomaly ${ }^{36}$. In the Finkelstein scenario, the Altshuler-Aronov effect leads to a reduced electron-phonon coupling constant through the increased electronic repulsion term, and thus to a reduced $T_{c}$ for increasing disorder.

On the other hand, Anderson localization effects alone induced by strong disorder (i.e. neglecting Coulombic effects) lead to the localization of singleelectron wavefunctions on a localization length $L_{l o c}$ ( $\sec ^{25}$ and references therein). This localization process introduces a new energy scale $\delta_{l o c}=1 /\left(\nu_{0} L_{l o c}^{3}\right), \nu_{0}$ being the DOS at $E_{F}$ per spin. $\delta_{l o c}$ represents the average electronic level spacing in a volume of size $L_{l o c}^{3}$ where electrons are localized. In principle, Cooper pairing of electrons in a localized volume is still possible if $\delta_{l o c}$ is smaller than $T_{c}$, as it is the case for single isolated small 

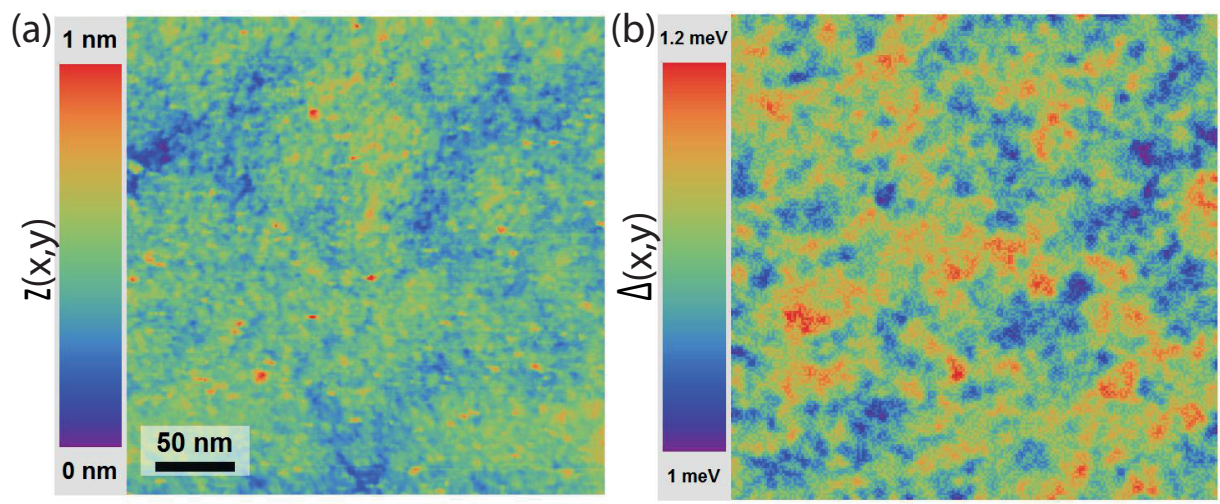

(e)
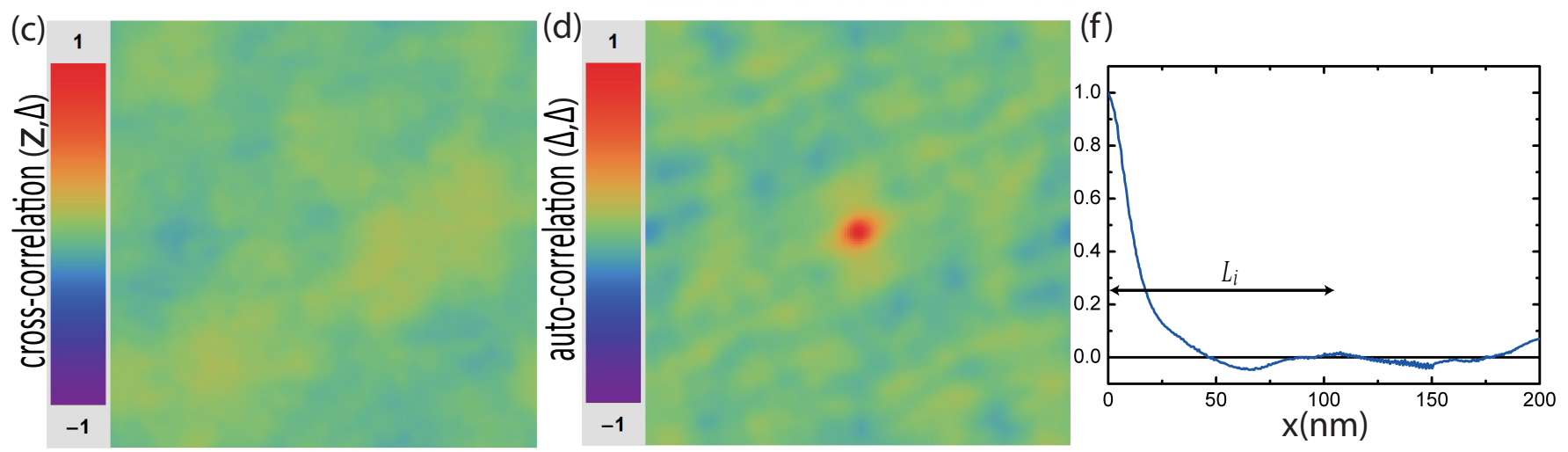

FIG. 3: Emergent granular superconducting properties in a so-called homogeneously disordered ultrathin film. $2.14 \mathrm{~nm}$ thick NbN film, probed by scanning tunneling spectroscopy. (a) Topographic map of the NbN area under study $\left(T_{c}=3.8 \mathrm{~K}\right)$. (b) Corresponding map displaying, by a color code, the spatial variations of the superconducting gap $\Delta$ measured at $T=300 \mathrm{mK}$. Gap inhomogeneities appear on a scale much larger than the size of the nanocrystals $\left(d_{g} \approx 2-5 \mathrm{~nm}\right)$ constituting the NbN films, seen in the topographic map and characterized by TEM. (c) Cross-correlation map between the topographic and spectroscopic maps revealing the absence of correlation. (d) Autocorrelation map of the gap map shown in (b). (e) Schematics of the relevant length scales. (f) Radial profile extracted from the autocorrelation map in (d). The correlation length $L_{i}$ is defined as the abscissa of the first peak away from the center and so is about $100 \mathrm{~nm}$ in the present sample. The central peak width, about $L_{i} / 2 \approx 50 \mathrm{~nm}$, gives an estimate of the typical domain size of constant gap values. This value is an order of magnitude larger than both the coherence length $\xi_{0}$ and the nanocrystal size $d_{g}$. Data from Carbillet et al. ${ }^{43}$ reproduced with permission.

grains $^{37,38}$. Thus, upon increasing disorder, a transition occurs to non-superconducting state when $\delta_{l o c} \simeq T_{c}$. This is the so-called Bosonic scenario. The resulting insulating state can thus in principle hosts localized Cooper pairs. In real systems the situation can be quite complex because both phenomena, Coulombic effects driving the Fermionic scenario and localization effects driving the Bosonic scenario, are usually simultaneously at play.

One fascinating feature discovered recently in these so-called homogeneous systems, using scanning tunneling spectroscopy (STS), is the emergence of granularity or inhomogeneity in their superconducting properties with increasing disorder, i.e. with $k_{F} l_{e}$ decreasing toward unity $^{39-43}\left(k_{F}\right.$ being the Fermi wave vector $k_{F} \leq 1.2 \AA^{-1}$ and $l_{e}$ the electronic mean-free-path typically less than few $\mathrm{nm}$ ). In terms of the film's square resistance, sizable effects start to happen when $R_{\text {square }}$ becomes larger than about $1 k \Omega$. It is observed that the superconducting gap measured in the local density of states (LDOS) by STS, presents at low temperature significant spatial variations on a length scale larger than the superconducting coherence length $\xi_{0}$. This effect is illustrated for a $3.6 \mathrm{~nm}$ thick TiN film in Fig. 2, whose characteristic resistivity and STS spectra are shown in Fig. 4 corresponding to the film TiN1.

Furthermore, one can wonder how these inhomogeneities are correlated to the disorder potential present in the film and ask ourselves whether these inhomogeneities are correlated to the distribution of nanocrystals constituting the films. This issue was addressed recently by Carbillet et $a l .{ }^{43}$. It was shown that such spatial gap variations are not correlated with the spatial distribution of $\mathrm{NbN}$ nanocystal constituting the films. This work is illustrated on a $2.14 \mathrm{~nm}$ thick $\mathrm{NbN}$ film studied over a $300 \times 300 \mathrm{~nm}^{2}$ area shown in Fig. 3a,b. The cross-correlation analysis, shown in Fig. 3c, is performed between the gap map presented in Fig. 3b and 
(a)

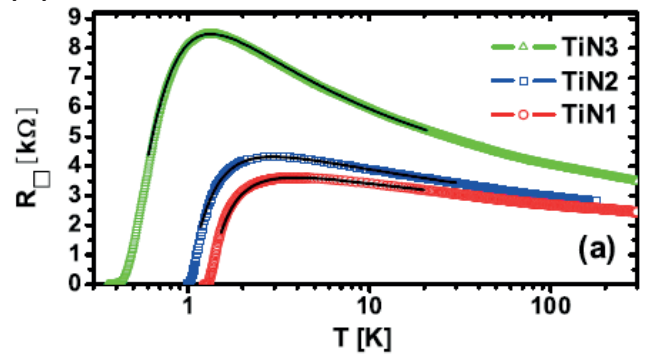

(b)

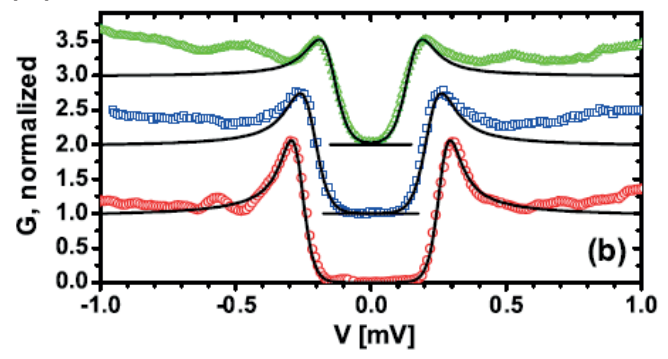

FIG. 4: Effect of increasing disorder on the local density of states of so-called homogeneously disordered ultrathin film. (a) Square resistance versus temperature for three ultrathin TiN samples of increasing disorder. (b) Normalized differential tunneling conductance measured by STS at $T=50 \mathrm{mK}$ (dots). The spectra are shifted for clarity. The BCS fits (solid lines) were calculated with the following parameters: TiN1: $\Delta=260 \mu \mathrm{eV}$ and an effective temperature $T_{\text {eff }}=0.25 \mathrm{~K}$; TiN2: $\Delta=225 \mu \mathrm{eV}$ and $T_{e f f}=0.32 \mathrm{~K}$; TiN3: $\Delta=154 \mu \mathrm{eV}$ and $T_{e f f}=0.35 \mathrm{~K}$. Data from Sacépé et al. ${ }^{39}$.

the topography seen in Fig. 3a. Fig. 3c shows no crosscorrelation $^{43}$. The relevant characteristic size of the domains of constant superconducting gap is extracted from the autocorrelation map of the gap map (Fig. 3d). The analysis shows that this size is about $50 \mathrm{~nm}$, an order of magnitude larger than both the coherence length $\xi_{0}$ (about $5 \mathrm{~nm}$ ) and the size $d_{g}$ (of about 2-5 nm) of the nanocrystals constituting the $\mathrm{NbN}$ film. A consequence of this work is that the superconducting inhomogeneities are not linked in a trivial way to the nano-structuration of the film.

Simultaneously, for increasing disorder levels the local $d I / d V$ spectra measured by STS start to deviate more and more from single-particle BCS excitation spectra, as found earlier in planar tunnel junctions ${ }^{33}$. This is illustrated in three ultrathin TiN films TiN1, Ti2, TiN3 of increasing disorder in Fig. 4b. Their corresponding square resistance versus temperature dependence is given in Fig. 4a. For low-disordered films (not shown here), $k_{F} l_{e} \gg 1, R_{\text {square }} \ll 1 \mathrm{k} \Omega$ and the $d I / d V$ spectra can be fitted using a BCS DOS without any additional broadening parameter. For smaller $k_{F} l_{e}$ values and $R_{\text {square }}$ larger than about $1 k \Omega$, the spectra show significantly smaller gap values, together with smaller and broader coherence peaks ${ }^{39-43}$. The tunneling spectra can be partially modelled using either the empirical Dynes parameter ${ }^{44}$ or alternatively a higher effective temperature as in Fig. $4 \mathrm{~b}$. At the same time a stronger and stronger AltshulerAronov background develops at $E_{F}$ affecting an energy range several orders of magnitude larger than the superconducting gap ${ }^{40,43}$. This latter effect makes it very hard to model and fit properly the experimental $d I / d V$ spectra, as there is no analytical formula how to include such electronic correlation effects in the excitation spectra, although there are some recent attempts ${ }^{45}$. For very large disorder the coherence peaks almost disappear ${ }^{46}$.

Another striking effect is found as the insulating (metallic) transition is approached further from the superconducting side. Above the critical temperature $T_{c}$ measured by transport experiment (usually defined as the temperature at which the zero resistance state is reached, but other operating definitions are also frequently used), a depleted DOS without coherence peaks remains at $E_{F}$ in the STS spectra over an energy scale comparable to the superconducting gap $\Delta$, and one recovers the normal DOS at significantly higher temperature ${ }^{40,43,46,47}$. This effect is referred to as a pseudogap, reminding the similarity of the observed effect with what is known from cuprates superconducting materials.

In addition to their fascinating basic properties, these systems attracted a lot of attention in the past fifteen years due to their chemical stability in air, their application in single-photon detectors or bolometers ${ }^{48}$ and their potential to realize high-kinetic inductance elements ${ }^{49}$.

\section{Crystalline ultrathin superconducting films}

In parallel to the works on disordered superconducting films reported above, a complementary field studying the superconducting properties of crystalline ultrathin films grown under ultrahigh vacuum (UHV) emerged in the last ten years ${ }^{50-56}$. These works were initiated by earlier studies in surface physics using MBE techniques. These new studies enabled extending earlier work in this direction $^{57,58}$. Crystalline films grown in UHV are much less disordered than granular or homogeneous disordered films presented above. Furthermore, their thickness can be controlled at the atomic level yielding high-quality macroscopic single-crystals. As a consequence crystalline films behave as very weakly disordered diffusive thin films hosting a perfect crystalline structure. For a given thickness their superconducting properties are thus homogeneous. The precise dependence of the superconducting properties as a function of film thickness could be studied in details ${ }^{50,52-56}$. Moreover, fine effects due to quantum confinement predicted earlier $^{59,60}$ were also investigated ${ }^{50,51,53}$. Let us note that we do not mention here the important new studies of the Berezinski-Kosterlitz-Thouless regime that will 
be presented later in section $\mathbf{V} \mathbf{D}$. The new interesting outcomes discovered during the studies of crystalline films are the following :

i) The superconducting properties are modulated by quantum-size effects due to the discrete vertical confinement produced by the integer number of monolayers constituting the film.

ii) The intrinsic behavior of the critical temperatureenergy gap and electron-phonon coupling constant could be studied as function of film thickness emphasizing the role of the surface and substrate.

iii) At the ultimate confinement, there exist several experimental systems consisting of a single plane of atoms grown on a semiconducting substrate that do superconduct and form a two-dimensional electron gas.

Most recent systematic studies were performed on lead $(\mathrm{Pb})$ films grown on silicon $\mathrm{Si}(111)$ substrate in UHV. This was made possible because silicon and lead are nonmiscible with each other, which results in an abrupt $\mathrm{Pb} / \mathrm{Si}$ interface. This simple fact combined with the remarkable property for lead that, twice the interatomic distance between the (111) dense $\mathrm{Pb}$ planes is almost commensurate with half the $\mathrm{Pb}$ bulk Fermi wavelength, $\lambda_{F} / 2 \sim 1.8 \mathrm{ML}$, have allowed researchers to grow both atomically smooth crystalline lead films over macroscopic distances and large nanoislands in a layer-by-layer way and study their local or macroscopic superconducting properties ${ }^{50,52-56}$.

The fabrication method is molecular beam epitaxy. First, the silicon substrate is prepared by direct current heating with several flashes up to $1200^{\circ} \mathrm{C}$, followed by an annealing from $900^{\circ} \mathrm{C}$ to $500^{\circ} \mathrm{C}$ during about 15 minutes. This leads to the so-called $\mathrm{Si}(111)-7 \times 7$ reconstruction of the atomic planes of the silicon surface. $\mathrm{Pb}$ is further evaporated on the freshly prepared $\mathrm{Si}(111)$ $7 \times 7$ substrate using either direct Joule heating with a filament or electron beam bombardment. For STS studies the deposition of $\mathrm{Pb}$ can be done on a substrate kept at room temperature, favoring the formation of flat-top islands ${ }^{61,62}$. These islands are single crystals and grow following a Stranski-Krastanov mode. A highly disordered $\mathrm{Pb}$ wetting layer covers first the entire $\mathrm{Si}(111)$ surface. Its thickness is about 2ML (the exact coverage of the wetting layer depends on the substrate temperature during the deposition). Pb Islands further grow, crystallizing directly on top of the silicon surface ${ }^{63}$. The $\mathrm{Pb}$ islands remain interconnected by the ultrathin $\mathrm{Pb}$ wetting layer. For macroscopic measurements like ARPES, electrical transport or magnetometry, the substrate has to be held below $130 \mathrm{~K}$ favoring a layer-by-layer growth. In this case all $\mathrm{Pb}$ layers are crystalline and the resulting sample is a single crystal. The precise thickness of the $\mathrm{Pb}$ islands or films, defined with respect to the top silicon atomic plane, is an exact

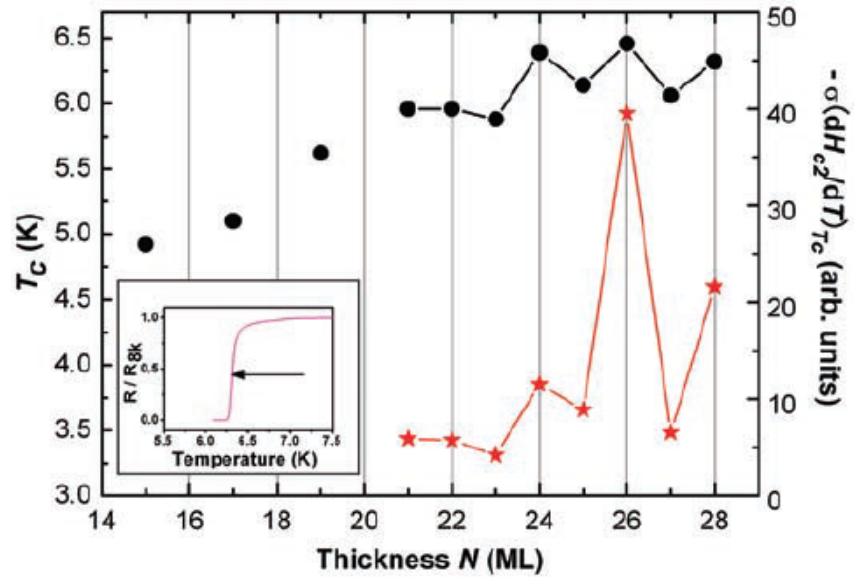

FIG. 5: Quantum-size effects on the critical temperature and density-of-states of macroscopically uniform monocrystalline $\mathrm{Pb}$ films measured ex situ. $\mathrm{Pb}$ films are grown in UHV on $\mathrm{Si}(111)$ and capped by a $4 \mathrm{ML} \mathrm{Au}$ protective layer to enable ex situ electrical resistivity measurements in perpendicular magnetic field. The films are macroscopically uniform and perfectly homogeneous, their thickness corresponding to an integer number $N$ of $\mathrm{Pb}$ monolayers (ML). The critical temperature $T_{c}$ is presented as a function of $N$ (left axis, dots). As shown in the inset, $T_{c}$ is extracted from the resistance value at half the normal state value. The density-of-states at the Fermi level is assumed to be proportionnal to the derivative of the perpendicular upper critical field $H_{c 2}$ with respect to the temperature $T$ evaluated at $T_{c}$ (right axis, stars). Oscillations between 23 and $28 \mathrm{ML}$ are due to vertical quantum confinement in the crystalline $\mathrm{Pb}$ films. Data reproduced from Guo et al..$^{50}$.

integer number of atomic $\mathrm{Pb}$ monolayers. This fact is demonstrated by the energy spectrum of quantum-well states confined in the direction perpendicular to the film measured by both ARPES and STS and further compared to ab initio calculations for free-standing $\mathrm{Pb}$ films ${ }^{64-68}$.

Because uniform $\mathrm{Pb}$ films can be prepared over macroscopic areas on $\mathrm{Si}(111)$ and their thickness can be controlled as an integer number of monolayers, Guo et al. could realize a tour-de-force and first revealed quantum-size effects on the superconducting properties of crystalline films ${ }^{50}$. In order to extract the critical temperature $T_{c}$ as a function of film thickness, they protected their films by a 4 MLs thick Au protective layer and performed ex situ electrical resistivity measurements in perpendicular magnetic field. Figure 5 shows that between 23 and 28 ML thickness, the critical temperature $T_{c}$ oscillates due to quantum-size effects with a $2 \mathrm{ML}$ periodicity. Between 20 and $15 \mathrm{ML}, T_{c}$ continuously decreases below $6.0 \mathrm{~K}$. The behavior of $T_{c}$ below $20 \mathrm{ML}$ is most probably affected by inverse proximity effect from the $\mathrm{Au}$ protective layer. The quantum oscillations in $T_{c}$ above $20 \mathrm{ML}$ are supported by concommitant os- 


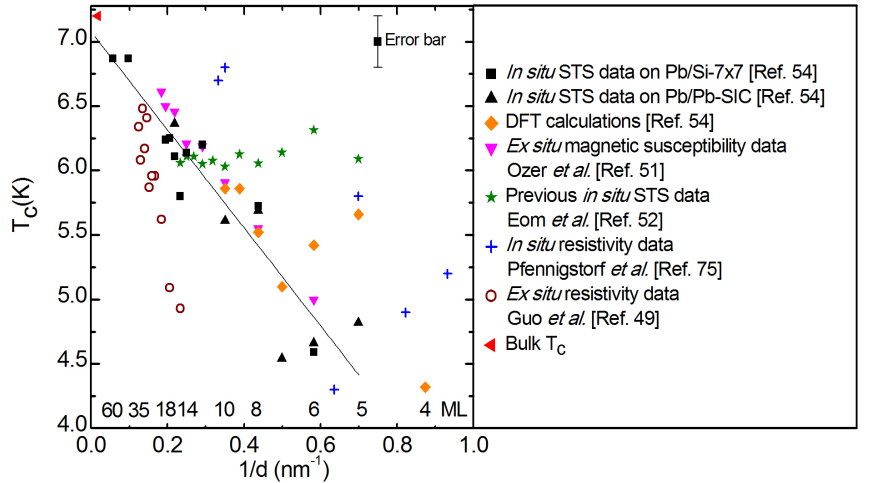

FIG. 6: Comparison of the critical temperature of crystalline $\mathrm{Pb}$ films as a function of the number of monolayers extracted from in situ or ex situ local or global measurements. The critical temperature $T_{c}$ is presented as a function of inverse film thickness $1 / d$ for monocrystalline $\mathrm{Pb}$ films grown in UHV on $\mathrm{Si}(111)$. In local STS measurements the gap was measured and $T_{c}$ was inferred assuming bulk $\Delta / k T_{c}$ ratio. For macroscopic measurement techniques, i.e. magnetic susceptibility measurements and in situ and ex situ transport, the $T_{c}$ is directly measured. Data reproduced from Brun et al. ${ }^{55}$.

cillations in the DOS at $E_{F}$ calculated for free-standing films and measured using the upper critical field (stars, right axis). The characterization of the films thickness is acertained by photoemission measurements of the energies of quantum-well states present in the studied $\mathrm{Pb}$ films. Interestingly quantum-size effects were found previously and later on to affect all physico-chemical properties of $\mathrm{Pb}$ films or islands, including their growth process and stable heights ${ }^{61,69-72}$, work function and surface energy ${ }^{68,73}$, perpendicular upper critical field ${ }^{51}$, chemical reactivity $^{74}$, calculated superconducting critical temperature $^{75}$ or Kondo temperature of adsorbed single magnetic molecules ${ }^{76}$ to mention a few.

The thickness-dependent superconducting properties were studied by ex situ ${ }^{50}$ and in situ electrical transport ${ }^{77}$, in situ $\mathrm{STS}^{53,55,56}$ and ex situ magnetometry ${ }^{52}$. STS results points to a BCS behavior of the local DOS down to few $\mathrm{MLs}^{53,55}$. The average trend of all data, although not performed on exactly the same systems because of the capping layers used in ex situ measurements, reveal that both the gap $\Delta$ and the critical temperature $T_{c}$ diminish with decreasing film thickness $d$ as $1 / d$. This is shown in Fig. 6. This reduction can be partly explained by $a b$ initio DFT calculations of the electron-phonon coupling constant $\lambda$ for $\mathrm{Pb}$ free-standing films, using the Migdal-Eliashberg theory. These calculations show indeed that $\lambda$ decreases with film thickness ${ }^{55,78}$, the reduction starting to be significant below $10 \mathrm{ML}$. Let us note that the Eliashberg function could be measured for $\mathrm{Pb}$ islands thicker than $10 \mathrm{ML}$ grown on $\mathrm{Cu}(111)$ by inelastic $\mathrm{STS}^{79}$. These experimental results agree with $a b$ initio DFT calculations. These measurements show an enhanced electron-phonon coupling constant when quantum-well states are close to $E_{F}{ }^{79}$, which is a quantum-size effect.

One can further wonder whether the oscillations of $T_{c}$, measured by ex situ transport measurements ${ }^{50}$ on $\mathrm{Pb}$ films which are due to quantum-size-effects, are also found in the in situ measurements of $\Delta$ by STS. A definite answer cannot be given yet as various studies performed at rather high temperatures lead to different results ${ }^{53,55}$. A more careful investigation at very-low temperature is needed in order to benefit from a higher energy resolution (possibly using a superconducting tip) and fully clarify this issue. Let us also note that a recent theoretical work shed new light on the theoretical treatment of quantum-size effects in BCS thin films ${ }^{80,81}$. In particular, Valentinis et al. have shown that, in contrast to previous calculations suggesting discontinuous jumps in $T_{c}$ as a function of film thickness due to discrete jumps in the DOS (see among others ${ }^{59,60,75}$ ), $T_{c}$ is in fact a smooth function of the film thickness ${ }^{80,81}$. They also found that depending on the confinement potential existing in the ultrathin film, $T_{c}$ can be reduced with respect to the bulk $T_{c}$, or be increased above the bulk $T_{c}$ before droping to zero for zero thickness. Key-ingredients in this treatment appeared to be the self-consistent adjustment of the chemical potential with varying thickness and the detailed consideration of finite well effects.

The second contribution which should also play an important role in reducing $\Delta$ and $T_{c}$, but has not yet been considered theoretically is the continuous reduction of the electron mean-free path $l_{e}$ as roughly twice the film thickness $l_{e} \approx 2 d^{52,82-85}$. The ultrathin crystalline $\mathrm{Pb}$ films are thus far in the diffusive regime since $l_{e}<<\xi_{0}$. In the diffusive regime the low-temperature coherence length $\xi_{0}$ is reduced much below the clean bulk value $\left(\xi_{\text {bulk }}=80 \mathrm{~nm}\right)$, according to the relation $\xi_{0}=\sqrt{\hbar D / \Delta}$, $D$ being the diffusion constant. Typically $\xi_{0} \approx 25 \mathrm{~nm}$ for a film (island) thickness $d$ about $4 \mathrm{~nm}$, as measured by STS from the size of the vortex cores $^{84,85}$. Thus one expects that for the thinnest $\mathrm{Pb}$ films, the Finkelstein mechanism described above in the section II B, could also contribute to reduce the gap and $T_{c}$. Finally the third effect that could also contribute to the reduction of $\Delta$ and $T_{c}$ is the effect produced by the silicon substrate. On one hand it could modify phonon modes and $\lambda$. On the other hand it could also have some effect on the effective density of states in the $\mathrm{Pb}$ films, as $\mathrm{Pb}$ first saturates all dangling bonds of the $\mathrm{Si}(111)-7 \times 7$ surface. These issues have to be studied further to get better theoretical and experimental clarifications.

\section{MONOLAYER FILMS GROWTH AND STRUCTURAL PROPERTIES}

The experimental work performed on monocrystalline $\mathrm{Pb} / \mathrm{Si}(111)$ ultrathin films presented above, in section II C, paved the way to the discovery in 2010 of super- 


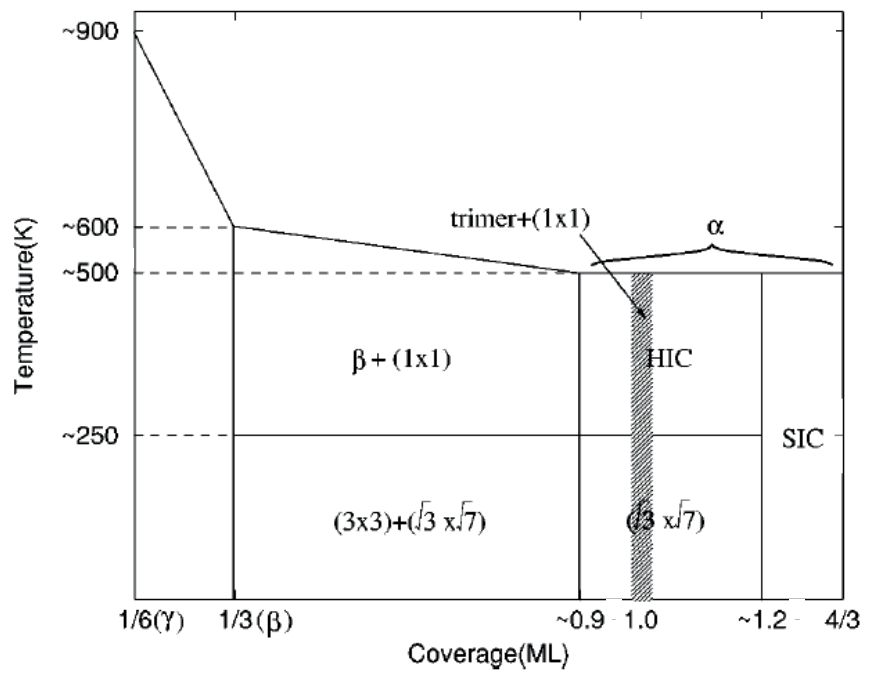

FIG. 7: Structural phase diagram of the $\mathrm{Pb} / \mathrm{Si}(111)$ monolayer system. Reported monolayer phases in the $\mathrm{Pb} / \mathrm{Si}(111)$ system for $\mathrm{Pb}$ coverage between $1 / 6$ and $4 / 3 \mathrm{ML}$. Reproduced from Chan et al. ${ }^{94}$.

conductivity in one monolayer of $\mathrm{In} / \mathrm{Si}(111)$ and two different $\mathrm{Pb} / \mathrm{Si}(111)$ crystalline monolayers by Zhang et al. ${ }^{8}$. In fact these systems were known for some time from the surface physics community to form crystalline monolayer phases ${ }^{86-92}$. The precise structure and coverage of each studied phase remained debated in the community. Nevertheless, thanks to low-energy electron diffraction (LEED), reflection high-energy electron diffraction (RHEED), STM, x-ray photoelectron spectroscopy (XPS) and surface x-ray scattering experiments (SXRD), the structural and electronic structure could be modelled and confronted to DFT calculations. There is now a rather large agreement on the phase diagram of the rich number of monolayer phases that exists in the $\mathrm{Pb} / \mathrm{Si}(111)$ system, as well as on the $\mathrm{Pb}$ coverage of these phases. Figure 7 shows this diagram in the coverage of interest $^{94,95}$.

The two peculiar phases in which Zhang et al. discovered superconductivity and which could be further studied by in situ electrical transport measurement (see section $\mathrm{V}$ below), are referred to as the $\sqrt{7} \times \sqrt{3}-\mathrm{Pb} / \mathrm{Si}(111)$ and Striped Incommensurate (SIC) $\mathrm{Pb} / \mathrm{Si}(111)$ phases. They correspond respectively to a coverage of $1.20 \mathrm{ML}$ and $1.30 \mathrm{ML}$. This coverage takes as reference the silicon atom density in the top $\mathrm{Si}(111)$ surface plane. These phases are grown by first preparing the $\mathrm{Si}(111)-7 \mathrm{x} 7$ reconstruction. Then a $\mathrm{Pb}$ quantity typically between 1.5 and 1.8 ML is deposited on the room temperature $\mathrm{Si}(111)$ $7 \mathrm{x} 7$ surface. An annealing between $500 \mathrm{~K}$ and $650 \mathrm{~K}$ is then performed to form either the SIC or $\sqrt{7} \times \sqrt{3}-\mathrm{Pb}$ phase. Let us note that a complex temperature dependent phase diagram exists for these two phases ${ }^{95}$, that we will not detail, as we are interested only in the very-low temperature phases of these systems, when they become

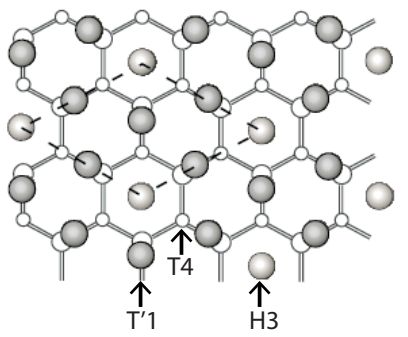

(b) $\sqrt{3} \times \sqrt{3}(1.33 \mathrm{ML})$ (d) $\quad \sqrt{7} \times \sqrt{ } 3(1.20 \mathrm{ML})$

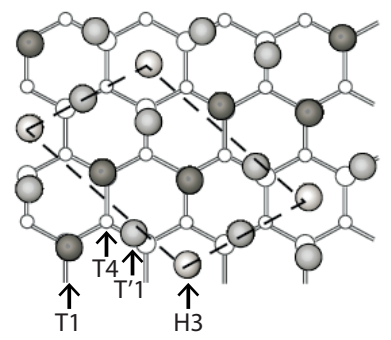

FIG. 8: Schematics of the structure models of two superconducting $\mathrm{Pb}$ monolayer phases grown at the surface. (a) The $\sqrt{7} \times \sqrt{3}-\mathrm{Pb} / \mathrm{Si}(111)$ corresponding to $1.20 \mathrm{ML}$ $\mathrm{Pb}$ coverage. (b) The $\sqrt{3} \times \sqrt{3}-\mathrm{Pb} / \mathrm{Si}(111)$ corresponding to the ideal coverage 1.33 ML. The large filled circles represent the surface $\mathrm{Pb}$ atoms. The large (respectively small) empty circles represent the silicon atoms of the first (resp. second) $\mathrm{Si}(111)$ plane. The characteristic adsorption site are indicated according to the standard nomenclature: T1 on-top site (above a Si atom of the first $\mathrm{Si}(111)$ plane), T'1 on-top site (slightly off-centered above a Si atom of the first $\mathrm{Si}(111$ ) plane), H3 hollow site, T4 (above a $\mathrm{Si}$ atom of the second $\mathrm{Si}(111)$ plane). Adapted from Choi et al. ${ }^{96}$.

superconducting.

First, it is interesting to note that the mismatch between the $\mathrm{Pb}$ and $\mathrm{Si}$ lattice constants, respectively of $4.92 \AA$ and $5.43 \AA$, is about $10 \%$. Thus, a commensurate $\sqrt{3} \times \sqrt{3}-\mathrm{Pb}$ overlayer phase on the $\mathrm{Si}(111)$ substrate would require the $\mathrm{Pb}$-overlayer to be compressed by $5 \%$. This is apparently too much and not observed. Nevertheless, the SIC phase is in fact very close from being a dense commensurate $\sqrt{3} \times \sqrt{3}$-Pb phase. While such truly commensurate $\mathrm{Pb}$ phase can be stabilized on $\mathrm{Ge}(111)$, the SIC remains incommensurate with a coverage about $1.30 \mathrm{ML}$, below $4 / 3$ corresponding to the coverage of the ideal $\sqrt{3} \times \sqrt{3}$ - $\mathrm{Pb}$. The atomic structures of the $\sqrt{7} \times \sqrt{3}$ - $\mathrm{Pb}$ and SIC phases are shown in Fig. 8 . $\mathrm{Pb}$ is thus compressed in both phases with respect to the $\mathrm{Pb}$ bulk lattice spacing in the (111) planes.

For the $\sqrt{7} \times \sqrt{3}$ - $\mathrm{Pb}$ phase there are $6 \mathrm{~Pb}$ atoms for 5 $\mathrm{Si}$ atoms in a single unit cell (u.c.). From SXRD experiments, it is inferred that the $\mathrm{Pb}$ monolayer is a rather flat, close-packed lead layer with six $\mathrm{Pb}$ atoms per unit cell ${ }^{91}$. Interatomic distances indicate that five lead atoms saturate all the dangling bonds of the silicon substrate with partially covalent bonds and stabilize the commensurate phase. Four of these lead atoms are almost in T1 ontop sites, one is located in a bridge position above two silicon atoms from different planes (i.e. in between $\mathrm{T} 1$ and T4 sites). The sixth lead atom is located on a hollow site H3, has no bonds to silicon atoms and can best be described by a split atomic position with a vertical separation of $0.6 \AA$. Combining SXRD $^{91}$ and $a b$ initio DFT calculations ${ }^{92}$, it could be inferred that 5 out $6 \mathrm{~Pb}$ atoms (the four off-centered T'1 sites and the bridge site) make covalent bonding to underlying Si atoms, saturating all Si dangling bonds. Furthermore DFT calculations 
(a)

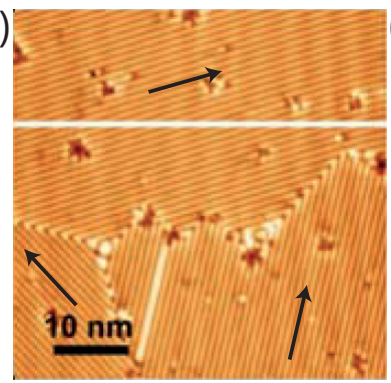

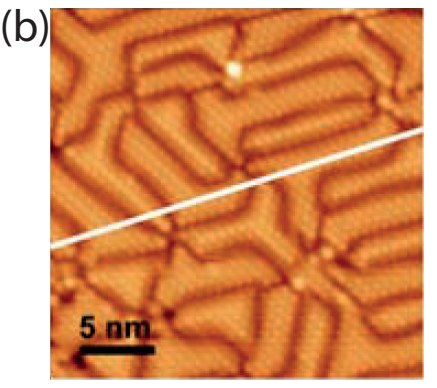

FIG. 9: Topographies of the dense superconducting $\mathrm{Pb} / \mathrm{Si}(111)$ monolayers measured by STM. The scale bar is indicated. (a) Area corresponding to three different domains, indicated by three arrows, of the linear $\sqrt{7} \times \sqrt{3}$ $\mathrm{Pb} / \mathrm{Si}(111)$ (Pb coverage: 1.20 ML ). (b) Meandering SIC phase $(\mathrm{Pb}$ coverage: $1.30 \mathrm{ML})$. Data from Zhang et al. ${ }^{8}$.

shows that metallic bonding exists between all $\mathrm{Pb}$ atoms, although atomic distances between nearest-neighbor $\mathrm{Pb}$ atoms vary due to bonding with $\mathrm{Si}$ atoms. As a consequence of the anisotropy of the commensurate unit cell, there are three equivalent rotational domains (rotated by $120^{\circ}$ ). It is thus not possible to grow a single crystal phase. The largest size of single domains is of the order of one hundred of nm. A small scale STM image showing the three orientations of the different domains is shown in Fig. 9a.

For the SIC phase, we instead represent the structure of the ideal $\sqrt{3} \times \sqrt{3}$ - $\mathrm{Pb}$ phase, which would have exactly $4 \mathrm{~Pb}$ atoms for $3 \mathrm{Si}$ atoms in a u.c. Three $\mathrm{Pb}$ atoms occupy off-centered T'1 sites, the remaining $\mathrm{Pb}$ atom occupying the $\mathrm{H} 3$ hollow site. This structure is termed the $\mathrm{H} 3$ structure. While this structure is in agreement with the experimental results reported above, DFT calculations not including spin-orbit coupling favor another structure, called T4 structure. Nevertheless, recent DFT calculations including spin-orbit coupling seem in agreement with the $\mathrm{H} 3$ structure being the lowest energy one $^{93}$.

The atomic structure of the real SIC phase is slightly less dense than the one of the ideal $\sqrt{3} \times \sqrt{3}$ - $\mathrm{Pb}$ phase, which can not be formed on a silicon substrate. It was shown that SIC phases are commensurate phases with a large unit cell which is built from the combination of $\sqrt{3} \times \sqrt{3}$ domains separated by meandering domain walls of the almost $\sqrt{7} \times \sqrt{3}$ phase (i.e. atoms at adjacent rows along the [-1-12] direction occupy H3-T4 sites instead of $\mathrm{H} 3-\mathrm{H} 3$ as in the regular $\sqrt{7} \times \sqrt{3}$ phase) $)^{87,88,90,94,95}$. A small scale STM image of the SIC phase is shown in Fig. 9b, with its characteristic meandering domain walls. The size of the $\sqrt{3} \times \sqrt{3}$ domains is small and typically of about $5 \mathrm{~nm}$, while the walls have a nm size.

\section{MONOLAYERS ELECTRONIC PROPERTIES FROM ARPES MEASUREMENTS CONFRONTED TO AB INITIO DFT CALCULATIONS}

Careful ARPES measurements were performed on monolayer phases with a well-defined and homogeneous $\mathrm{Pb}$ coverage, characterized by an homogeneous LEED or RHEED pattern. The detailed Fermi surfaces and bandstructure could be mapped out for both phases. Instead of the exact SIC, a close linear phase of the devilstaircase phases was chosen for its better LEED homogeneity (coverage 1.28 ML). For this latter phase, a slightly miscut $\mathrm{Si}(111)$ substrate even allowed a monodomain phase to be grown, while for the $\sqrt{7} \times \sqrt{3}$ phase the three rotational domains were always present ${ }^{96}$.

Free-electron like Fermi contours were revealed in both systems $^{96}$. The overall apparent periodicities found from ARPES are drastically different for the two phases. For the $\sqrt{7} \times \sqrt{3}$ phase it is found that the $1 \times 1$ periodicity produced by the $\mathrm{Si}(111)$ surface structure dominates, while a $\sqrt{3} \times \sqrt{3}$ periodicity dominates for the SIC phase. These results are very striking for the $\sqrt{7} \times \sqrt{3}$ phase, because there seems, at first, to be no direct signatures of a $\sqrt{7} \times \sqrt{3}$ periodicity in the electronic bandstructure, while in electron or photon diffraction experiments such signatures are obvious.

Contours of the Fermi surface of the $\sqrt{7} \times \sqrt{3}$ phase are shown in Fig. 10a. These complicated contours correspond to the green triangles and blue hexagons seen in Fig. 10b, being respectively electron and hole pockets. It is visible that these blue and green lines lye all on the dashed red circles, centered on the reciprocal lattice vectors of the $\mathrm{Si}(111)$ surface closest to the $\Gamma$ point (denoted $\left.\Gamma_{01}, \Gamma_{10} \ldots\right)$. The red circles correspond to an array of $2 \mathrm{D}$ free-electron parabolas, of radius $k_{F} \approx 1.36 \AA^{-1}$. Fig. 10c shows the corresponding electron bands dispersion along the characteristic high-symmetry points of the $\mathrm{Si}(111)$ surface Brillouin zone. This allows to extract a Fermi energy of about $0.8 \mathrm{eV}$ and an effective mass of about $1.16 m_{e}$ ( $m_{e}$ being the free-electron mass).

With the same scheme as for the $\sqrt{7} \times \sqrt{3}$, the Fermi contours and bands dispersion of the SIC phase are shown on Fig. 10d,e,f. Nice hexagons, corresponding to the orange contours, are seen. They follow directly the $\sqrt{3} \times \sqrt{3}$ periodicity and are repeatedly centered on each equivalent $\Gamma^{\prime}$ points defining the $\sqrt{3} \times \sqrt{3}$ reciprocal lattice. According to Choi et al., the effect produced by the linear long-range periodicity of the devil staircase phase, due to the mixing of mostly $\sqrt{3} \times \sqrt{3}$ periods and few $\sqrt{7} \times \sqrt{3}$ periods, is seen along the $\Gamma^{\prime}-M^{\prime}$ direction : it splits the hexagons in segments parallel to the $\Gamma_{00}-\Gamma_{01}$ direction. The electron bands shown in Fig. 10f are dispersing parabolically. It is concluded from these studies that in the SIC phase, the electrons mostly see a large $\sqrt{3} \times \sqrt{3}$ periodic potential. Additionally, the electrons feel a weak long-range potential (uni- or multidirectionnal) giving the whole incommensurate 

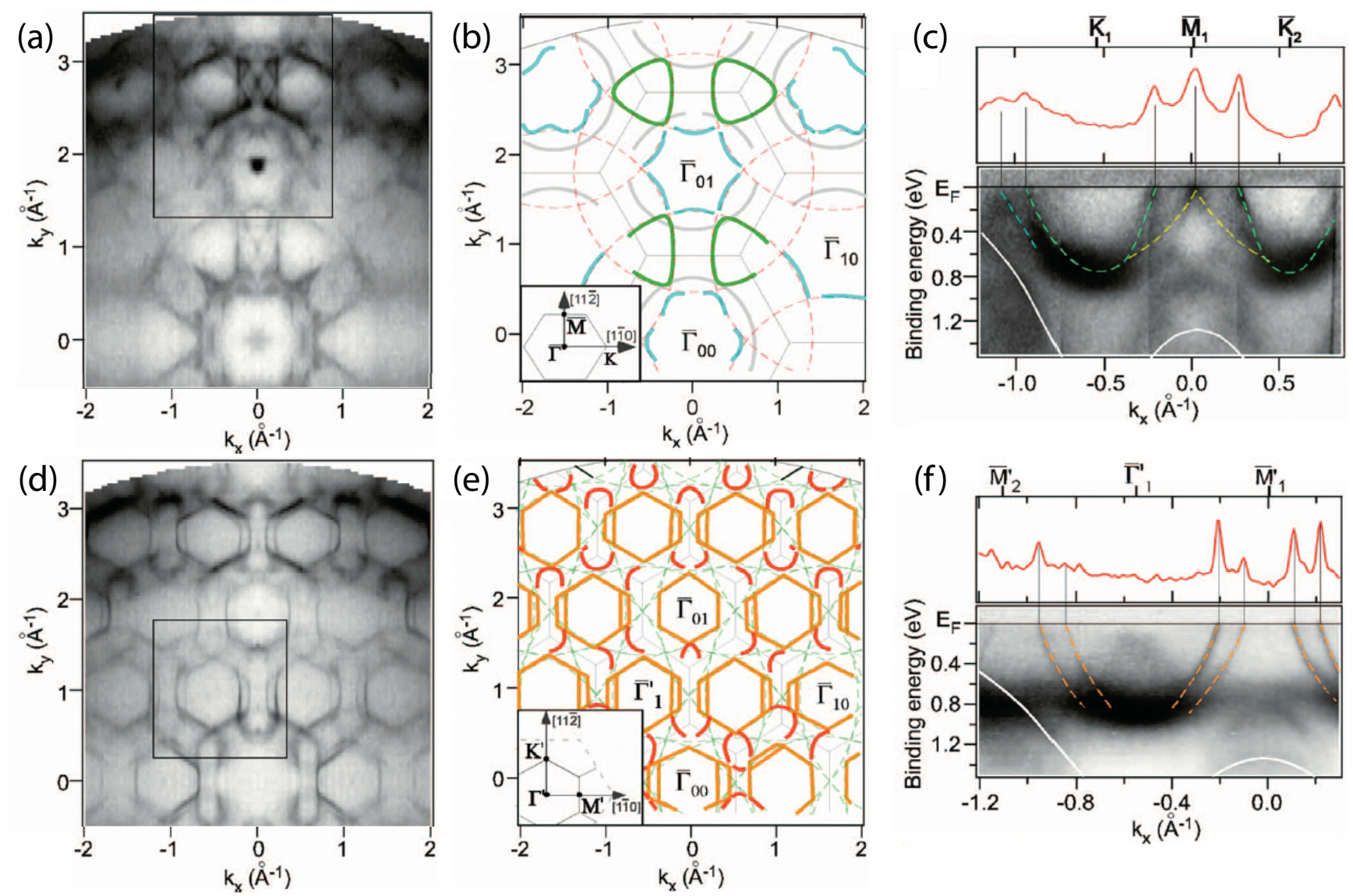

FIG. 10: ARPES measurements of the Fermi surface and bands dispersion of dense superconducting $\mathrm{Pb} / \mathrm{Si}(111)$ monolayers. Fermi contours and band dispersions measured by ARPES, and their schematic illustrations for the $\operatorname{Si}(111) \sqrt{7} \times$ $\sqrt{3}-\mathrm{Pb}$ (a,b,c panels) and $\mathrm{Si}(111) 14 \times \sqrt{3}-\mathrm{Pb}$ phase $(\mathrm{d}, \mathrm{e}, \mathrm{f}$ panels $)$. The $14 \times \sqrt{3}-\mathrm{Pb}$ phase is representative of the SIC phase as their coverages are very close. (a,d) Photoelectron intensity map at $E_{F}$ in $k_{\|}$space taken with a photon energy of $90 \mathrm{eV}$. The intensity is given in a gray scale with higher intensity for darker contrast. That is, a dark part of this map corresponds to an $E_{F}$ crossing of a metallic band. (b,e) Schematic illustration of the traces having dark features (the Fermi contours) observed in $(\mathrm{a}, \mathrm{d})$. The thick solid lines indicate the different Fermi contours, as explained in the text. The thin solid lines depict the $1 \times 1$ Surface Brillouin Zone (SBZ), whose details are given in the inset. The dashed circles are free-electron Fermi contours centered on each $1 \times 1$ SBZ. (c,f) Energy versus momentum maps of the photoelectron intensity along the $K_{1}-M_{1}-K_{2}$ SBZ line and $M_{2}^{\prime}-\Gamma_{1}^{\prime}-M_{1}^{\prime}$. The momentum distribution curves at $E_{F}$ are also plotted on the top. The white solid lines indicate the upper bound of the Si bulk band. Adapted from Choi et al. ${ }^{96}$.

period of the SIC, needed to accomodate the strain induced by the many $\sqrt{3} \times \sqrt{3}$ periods released by short-ranged $\sqrt{7} \times \sqrt{3}$ domain walls. For the SIC, $k_{F}$ is slightly larger than for the $\sqrt{7} \times \sqrt{3}: k_{F} \approx 1.44 \AA^{-1}$, the Fermi energy being also a bit larger, close to $0.9 \mathrm{eV}$, with $m$ about $1.27 m_{e}$.

A refined ARPES experiments of the Fermi surface, togoether with ab initio DFT calculations, was achieved for the $\sqrt{7} \times \sqrt{3}$ phase $^{97,98}$. This is illustrated in Fig. 11. This allowed to shed new light on the puzzling results reported above. The calculated constant energy contours at $E_{F}$ for the (a) single domain and (b) triple rotational domains surface are seen. The $\Gamma, \mathrm{M}$, $\mathrm{K}$ points and dashed hexagon refer to the $1 \times 1 \mathrm{Si}(111)$ first-Brillouin-zone. The thin elongated hexagons along the $\Gamma-M$ direction refer to the $\sqrt{7} \times \sqrt{3}$ reciprocal unit cells. The analysis demonstrates in Fig. 11c that a very good agreement is obtained between DFT calculations and the measured ARPES Fermi surface in the $1 \times 1$ surface first-Brillouin-zone, when the existence of triple rotational domains is taken into account. Thus, this refined analysis enables demonstrating that for a $\sqrt{7} \times \sqrt{3}$ single-domain, the real electronic structure and Fermi surface does in fact reflect the $\sqrt{7} \times \sqrt{3}$ periodicity, as seen by the large warped parallel Fermi sheets denoted $\mathrm{S} 2$ in Fig. 11a. However, because of the existence of rotational domains over the surface, the resulting features measured by ARPES reflect an effective $1 \times 1$ periodicity.

In terms of electronic orbitals contribution, DFT calculations show that the superconducting behavior of the dense $\mathrm{Pb} / \mathrm{Si}(111)$ monolayer is almost entirely due to $\mathrm{Pb} 6 p_{x, y}$ orbitals, indicating in-plane $\mathrm{Pb}-\mathrm{Pb}$ metallic 
(a)

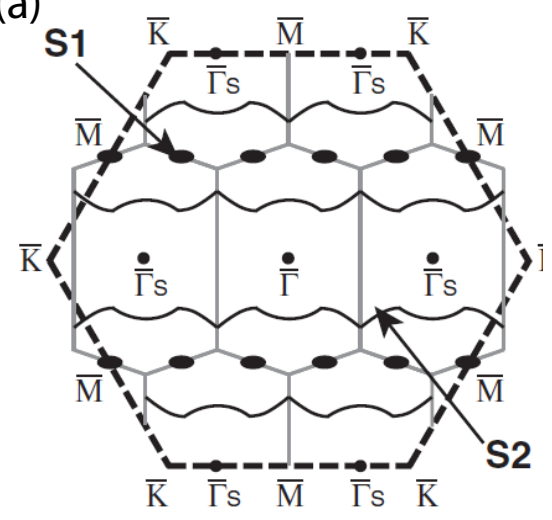

(b)

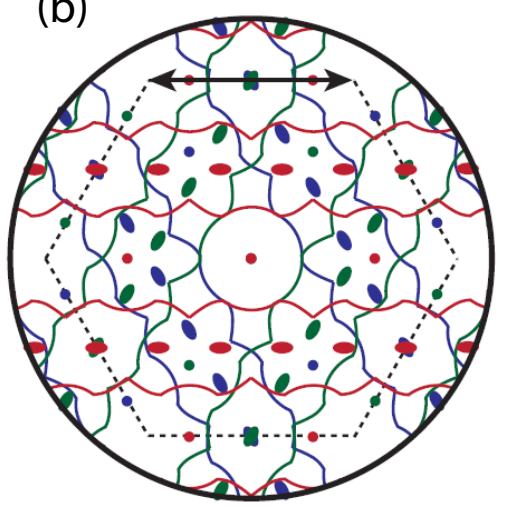

(c)

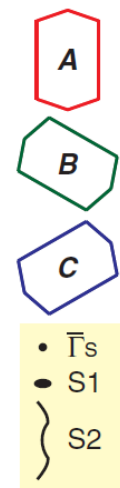

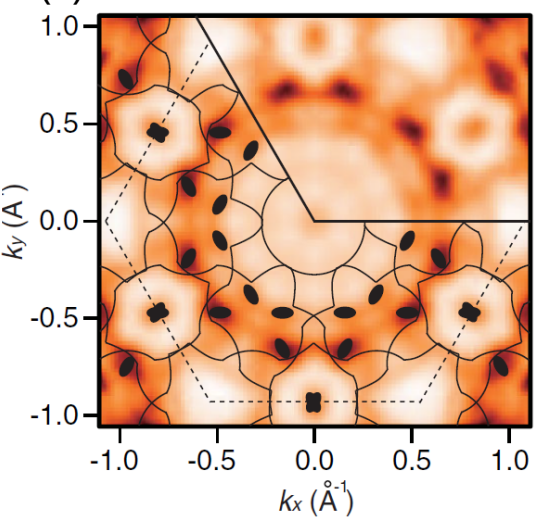

FIG. 11: Refined ARPES experiments of the $\sqrt{7} \times \sqrt{3}-\mathrm{Pb} / \mathrm{Si}(111)$ phase confronted to ab initio calculations. Constant energy contours at $E_{F}$ calculated from DFT for the (a) single-domain and (b) triple rotational domains surface. Thin continuous grey (thick dashed black) lines represents the surface unit cell for the $\sqrt{7} \times \sqrt{3}$ - $\mathrm{Pb}$ phase (resp. for the $1 \times 1$ $\mathrm{Si}(111)$ surface). Contours from each domain (A, B, and C) are indicated by different colors in (b). (c) ARPES constant energy contours at $E_{F}$. The DFT contours from (b) are superimposed. The raw data (region without DFT contours) are symmetrized reflecting the fundamental mirror symmetry. Adapted from Kim et al. ${ }^{97}$.

bonding around $E_{F}$. Deeper-bound dispersionless bands have a covalent $\mathrm{Pb} 6 p_{z}$ and $\mathrm{Si} 3 p_{z}$ character, indicating covalent $\mathrm{Pb}-\mathrm{Si}$ bonding. These bands are however completely filled and thus do not participate to the Fermi level properties. Interestingly, the comparison between the free-standing $\mathrm{Pb}$ monolayer and the $\mathrm{Pb} / \mathrm{Si}(111)$ monolayer shows that in the real $\mathrm{Pb} / \mathrm{Si}(111)$ system, the metallic states with $p_{x, y}$ character are effectively decoupled from the substrate ${ }^{98}$.

An important point to notice is that the reported DFT calculations do not include spin-orbit coupling in the $\mathrm{SIC}$ and $\sqrt{7} \times \sqrt{3}$ - $\mathrm{Pb}$ phases. Although nice agreement exist with ARPES measurements, this is clearly a backdraw as recent experimental results suggest that spin-orbit coupling plays an important role for superconductivity, as will be presented in sections $\mathrm{VC}$ and VIB. New DFT calculations of the ideal $4 / 3$ monolayer $\sqrt{3} \times \sqrt{3}-\mathrm{Pb} / \mathrm{Si}(111)$ and $\mathrm{Ge}(111)$ including spin-orbit coupling have just been performed. They favor the H4 structure model and show the existence of a large Rashba spin-splitting of the $\mathrm{Pb}$ surface states $^{93}$. More work is needed to compare in details the obtained electronic band structure to available experimental results and ascertain the various effects produced by spin-orbit coupling.

In conclusion, ARPES experiments support a 2D nearly-free electron picture compatible with the structural models developed in the section III for the SIC and $\sqrt{7} \times \sqrt{3}$ phases. The electronic bands in both phases share a similar origin, although their characteric surface potential is different. The number of in-plane electrons populating the measured valence bands, estimated per $1 \times 1$ unit cell, is about 3.8 for the $\sqrt{7} \times \sqrt{3}$ and 4.2 for the SIC. This is consistent with each $\mathrm{Pb}$ atoms involving 4 valence electrons. More work is needed to characterize in details the effects of spin-orbit coupling and Rashba effect at the surface on the electronic band structure measured by ARPES, using for instance spin-resolved ARPES.

\section{SUPERCONDUCTING PROPERTIES FROM IN SITU MAGNETO-TRANSPORT MEASUREMENTS}

\section{A. General transport properties in perpendicular magnetic field}

While STS reported superconductivity in $\mathrm{Pb}$ and In monolayers in 2010, great experimental achivements reported macroscopic superconductivity from in situ 4point electrical resistivity measurements performed in UHV. This was first done in $\sqrt{7} \times \sqrt{3}-\mathrm{In} / \mathrm{Si}(111)$ in $2011^{99}$ and in $\mathrm{SIC} \mathrm{Pb} / \mathrm{Si}(111)$ in $2013^{100}$. These achievements are very important for the field of one atom-thick superconductors, because one could have expected that superconductivity could be able to develop locally inside flat terraces but would be stopped at step edges. If this would occur, a macroscopic supercurrent could not propagate across a real macroscopic sample, made of numerous atomic steps in-between the voltage probe electrodes.

The techniques used by Uchihashi et al. and Yamada et al. are different. As shown in Fig. 12a, Uchihashi et al. patterned their sample in a macroscopic Van-der-Pauw square configuration by etching the prepared $\sqrt{7} \times \sqrt{3}$-In/Si(111) monolayer with $\mathrm{Ar}^{+}$sputtering through a shadow mask. The electrical transport properties were further measured by contacting the four delimited $1 \times 1 \mathrm{~mm}^{2}$ squares with 4 gold-coated springs. Yamada et al. used a linear micro-four-point-probe device with a probe spacing of 20 microns to directly contact the 
sample without any mask ${ }^{101}$. Both groups characterized by LEED or RHEED their phases prior to transport measurements. To ensure that all the current is carried only by the metal overlayers, these groups used low-doped Si substrates or even intrinsic ones. The experimental transport characteristics are shown in Fig. 12b,c.

A superconducting transition is observed leading to a zero-resistance state for $T_{c} \approx 2.8 \mathrm{~K}$ for the $\sqrt{7} \times \sqrt{3}$ $\mathrm{In} / \mathrm{Si}(111)$ and $1.1 \mathrm{~K}$ for the $\mathrm{Pb} \mathrm{SIC}$ phase. We have not detailed in this review the structural and electronic properties of the In/Si(111) monolayers as they are less well known and studied than the $\mathrm{Pb}$ phases. There is for example still a controversy regarding the actual In coverage of these monolayers, while this issue is already settled for $\mathrm{Pb}$ phases. According to Uchihashi et al. ${ }^{99}$ and previous work ${ }^{102}$ the coverage of the $\sqrt{7} \times \sqrt{3}-\operatorname{In} / \operatorname{Si}(111)$ reconstruction was believed to be one-atom-thick. More recent ab initio DFT calculations show that this coverage should actually correspond to a double-layer ${ }^{103,104}$, although metallic single-layers may also exist ${ }^{105}$. Nevertheless, Yamada et al. confirmed the in situ transport results of Uchihashi et al., and could determine that in fact two different $\sqrt{7} \times \sqrt{3}$-In ML phases, the rectangular and hexagonal ${ }^{102}$, are superconducting with slightly different $T_{c}$ (of respectively $2.8 \mathrm{~K}$ and $2.4 \mathrm{~K}$ ).

For these systems, the square resistance at $5 \mathrm{~K}$ before the transition is of $410 \Omega$ for the In phase and $1.3 \mathrm{k} \Omega$ for the $\mathrm{Pb}$ SIC. Thus one clearly sees that, with respect to the general properties of homogeneous superconducting films presented above in section II B, the studied monolayer phases are far from being strongly disordered and far from the insulating or metallic transition. From the knowledge acquired in the field of disordered homogeneous thin films, one should thus expect only weak disorder effects.

In contrast to a sharp superconducting transition, it can be seen in Fig. 12b,c that the resistance starts to decrease much above $T_{c}$. This effect is well-known in ultrathin films and could be analyzed further by Yamada et al. $:$ it is ascribed to superconducting fluctuation effects ${ }^{106}$. The $R_{\text {square }}(T)$ curve could be satisfactorily fitted taking into account only an Aslamasov-Larkin term and a relevant anomalous Maki-Thomson term ${ }^{107}$ (continuous curve in Fig. 12c).

The evolution of the electrical transport properties was studied as a function of perpendicular magnetic field by Yamada et al.. From the Ginzburg-Landau (GL) theory, superconductivity is suppressed for the zero-temperature upper critical field $H_{c 2}=\Phi_{0} /\left(2 \pi \mu_{0} \xi_{G L}(0)^{2}\right)$, where $\Phi_{0}$ is the flux quantum and $\xi_{G L}(0)$ is the GL zero-temperature coherence length. An approximate linear behavior of $H_{c 2}(T)=H_{c 2}(0)\left(1-T / T_{c}\right)$ was found and enabled the estimation of $B_{c 2}=\mu_{0} H_{c 2} \approx 0.1 \mathrm{~T}$ for the SIC phase.

Combining ARPES and electrical transport results one can estimate the electronic elastic mean free path for the SIC phase. From the expression of the diffusion coefficient $D, D=v_{F} l_{e} / 2$ in two-dimensions $\left(v_{F}\right.$ being the Fermi velocity), one has at zero temperature $\xi=\sqrt{\xi_{0} l_{e}}$ $=\sqrt{\hbar D / \Delta}$. We estimate $v_{F}$ from the values quoted in section IV, $v_{F}=\hbar k_{F} /\left(1.27 m_{e}\right), v_{F} \approx 10^{-6} \mathrm{~m} . \mathrm{s}^{-1}$. Thus from $\xi \approx \xi_{G L} \approx 74 \mathrm{~nm}$, we get $l_{e} \approx 4 \mathrm{~nm}$ and $k_{F} l_{e} \approx 60$. As $k_{F} l_{e} \gg 1$, these values again testify for the $\mathrm{Pb}$ monolayers being very far from any emergent granular superconducting properties, which should appear when $k_{F} l_{e}$ becomes significantly smaller than 10 , as reported in section IIB and found by STS in the works ${ }^{39-43}$. These conclusions should also hold for the In monolayers since their normal state square resistances before the superconducting transition are even smaller than the $\mathrm{Pb}$ ones.

Another intriguing heterogeneous monolayer system prepared under UHV has revealed superconductivity in $2015^{108}$. It consists in a single layer of thallium grown on $\mathrm{Si}(111)-7 \times 7$, forming a $(1 \times 1)$ reconstruction. By itself this system is not metallic at the surface but metallic surface states are easily induced by depositing $1 / 3 \mathrm{ML}$ of $\mathrm{Pb}$ atoms on top of the $(1 \times 1)-\mathrm{Tl} / \mathrm{Si}(111)^{109}$. The $\mathrm{Pb}$ atoms form a $\sqrt{3} \times \sqrt{3}$ surface unit cell sitting on $\mathrm{T} 4$ sites. This system becomes superconducting with a $T_{c}=2.25 \mathrm{~K}$ and a square resistance at $5 \mathrm{~K}$ of about $300 \Omega$. The reported dependences of the square resistance as a function of temperature and perpendicular magnetic field are very similar to the ones reported in 100 for the SIC-Pb monolayer, leading to $B_{c 2}=0.67 \mathrm{~T}$ and $\xi_{G L}(0)=22 \mathrm{~nm}$.

\section{B. Critical current and role of atomic steps}

The critical current $I_{c}$, i.e. the maximum supercurrent that the superconducting monolayers can carry, was studied as a function of temperature by Uchihashi et al.. Its dependence is shown by the blue squares on Fig. 13. The maximum current density at the lowest achievable temperature in the experiment of $1.8 \mathrm{~K}$ is $J_{2 D, c}=0.018 \mathrm{~A} . \mathrm{cm}^{-1}$. This corresponds to $J_{3 D, c}=$ $6.1 \times 10^{5} \mathrm{~A} . \mathrm{cm}^{-2}$, assuming as expected that all the current flows only through the thickness of the In monolayer. This value is remarkably high for a one-atom-thick film.

Furthermore, a very interesting result is deduced from the temperature dependence of $I_{c}(T)$. The critical current density is not determined by Cooper pair breaking in the interior of the monolayer, a behavior which would lead to the dashed red curve not following the experimental points. The continuous blue curve shows that $I_{c}(T)$ is much better described assuming that Josephson junctions exist in the In monolayer and determine the maximum supercurrent ${ }^{110}$. Uchihashi et al. proposed that these Josephson junctions are formed by atomic step edges distributed all along the surface. The only remaining parameter of the Ambegaokar-Baratoff temperature dependence of the critical current allows extracting the normal resistance (for a unit of length) of a single atomic step. The value found, $\rho_{\text {step }}=3.310^{-4} \Omega$.m is in agreement with single step resistances reported in other metallic monolayers by in situ UHV 4-probe measurements ${ }^{111}$. A better quantitative model for $I_{c}(T)$ should be searched 
(a)

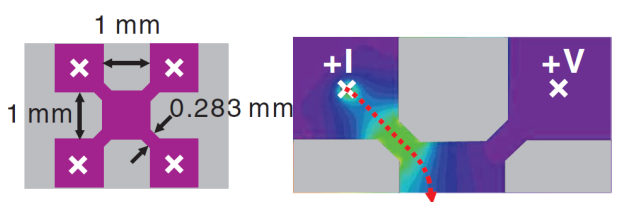

(b)

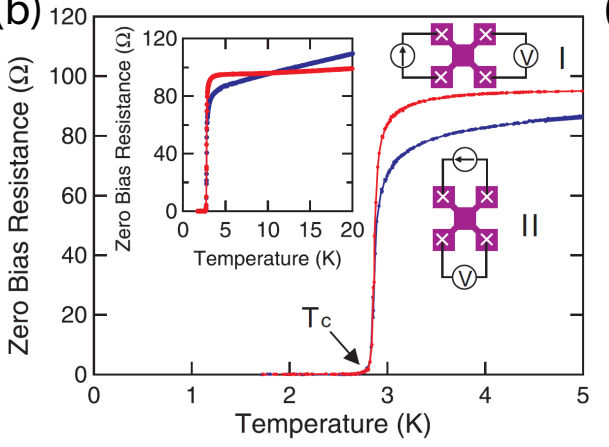

(c)

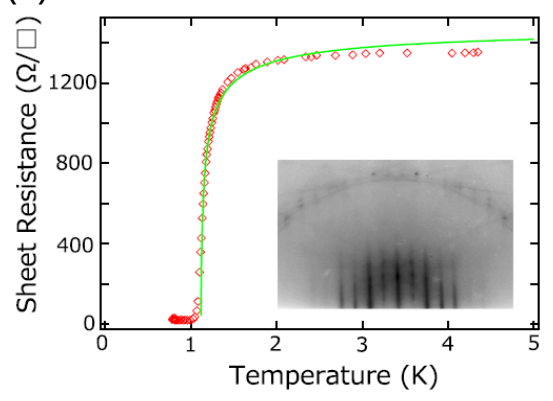

FIG. 12: In situ transport measurements of $\mathbf{P b}$ and In monolayers grown at silicon surface. (a) Drawing of the in situ sample patterning. The central and the four outer squares (purple areas) are made of $\sqrt{7} \times \sqrt{3}-\mathrm{In} / \mathrm{Si}(111)$ grown in UHV, and the surrounding regions (gray areas) consist of in situ sputtered Si surfaces. The right panel shows the calculated current density distribution on the sample. The bright green (dark purple) represents a high (low) current density. The red dotted line indicates the flow of a bias current. (b) in situ measured temperature dependence of zero bias resistances. $R_{0, I}$ (red lines) and $R_{0, I I}$ (blue lines) are zero bias resistances measured using the probe configurations I and II, respectively, with dc bias currents of $1 \mu \mathrm{A}$. Left inset: $R_{0, I}$ and $R_{0, I I}$ for a larger temperature range. Right insets: schematic drawings of the probe configurations I and II. (c) in situ measured temperature dependence of the square resistance of the Si(111)SIC-Pb monolayer grown in UHV, with the inset showing the RHEED pattern. The solid line is the result of a least-square fit to model superconducting fluctuations effects. Note that there is a controversy regarding the actual In coverage of the $\sqrt{7} \times \sqrt{3}-\mathrm{In} / \mathrm{Si}(111)$ system, between a monolayer according to previous work ${ }^{8,99,102}$ and a double-layer according to recent DFT calculations ${ }^{103,104}$. Panels a) and b) are adapted from Uchihashi et al. ${ }^{99}$ and c) from Yamada et al. ${ }^{100}$.

for by modelling the real monolayer with its numerous atomic steps not as a single Josephson junction but as an array of Josephson junctions.

The hypothesis that single atomic steps behave as Josephson barriers for one-atom-thick superconductors, deduced by Uchihashi et al. in the $\sqrt{7} \times \sqrt{3}-\operatorname{In} / \operatorname{Si}(111)$ monolayer from the peculiar temperature dependence of the critical current, was found to be demonstrated later by local STS experiments in perpendicular magnetic field on $\sqrt{7} \times \sqrt{3}-\mathrm{Pb} / \mathrm{Si}(111)^{112}$ and $\sqrt{7} \times \sqrt{3}-\mathrm{In} / \mathrm{Si}(111)^{113}$. Interestingly, such a behavior does not occur in the $\mathrm{Pb}$ SIC system. This means that in the SIC monolayer neighboring atomic terraces are electronically much better connected to each other ${ }^{112}$. This will be presented and further discussed togoether with STS results in section VI below.

\section{C. magneto-transport measurements in parallel magnetic field : hint for a spin-triplet component of the order parameter}

While so far experiments on $\mathrm{Pb}$ or In superconducting monolayers were compatible with having a conventional $s$-wave order parameter ${ }^{8,99,100}$, recent magneto-transport measurements in high parallel magnetic field seriously modify this picture ${ }^{114}$. Sekihara et al. have studied monolayer thick $\mathrm{Pb}$ films grown under UHV at helium temperature on an in situ cleaved GaAs(110) substrate. The crystalline structure of the films could not be characterized in situ. The comparison with the growth of $\mathrm{Pb}$ films on $\mathrm{Si}(111)$ or $\mathrm{Ge}(111)$ substrates strongly suggest that the $\mathrm{Pb} / \mathrm{GaAs}(110)$ should be structurally highly dis- ordered and most probably amorphous, with a structure similar to the case of the wetting layer of $\mathrm{Pb} / \mathrm{Si}(111)$. Nevertheless, the deposited $\mathrm{Pb}$ content of about $1 \mathrm{ML}$ was carefully established with a quartz microbalance, enabling a precise characterization of the surface density of $\mathrm{Pb}$ atoms.

An important difference between the Si(111) (or $\mathrm{Ge}(111)$ ) and the $\mathrm{GaAs}(110)$ surface is the absence of surface states in the bulk gap energy region in the latter case. There might be thus much less direct bonding between $\mathrm{Pb}$ and surface $\mathrm{Ga}$ or As atoms than with Si ones, which satisfactorily explains why a $1 \mathrm{ML} \mathrm{Pb}$ content is enough to reach metallic conductivity on $\operatorname{GaAs}(110)$. The in situ magneto-transport measurements in high parallel magnetic field, for an atomic surface density of $7.2 \mathrm{~nm}^{-2}$, are shown in Fig. 14. This surface density is less than the $\mathrm{Pb}(111)$ surface density $\left(9.4 \mathrm{~nm}^{-2}\right)$ and SIC-Pb density $\left(10.4 \mathrm{~nm}^{-2}\right)$. It is seen that a high-parallel field of $13.7 \mathrm{~T}$ has a very weak effect on the temperature dependence of the monolayer square resistance $R_{s q}(T)$ : this experimental curve is almost identical to the zero-field curve.

The groundstate of a conventional superconductor is a superposition of coherent doubly-occupied and doublyempty single electron states having opposite crystalline momenta and spins. For such ultrathin $s$-wave superconductors, the effect of a parallel magnetic field is to Zeeman split the density of states of single-electron states for spin parallel and anti-parallel to the applied field. No orbital depairing effect occurs. As a consequence superconductivity survives until the Pauli paramagnetic limit is reached ${ }^{115,116}$. The critical field at $T=0 \mathrm{~K}$ is then given by $H_{c}=H_{P a u l i}=\Delta_{0} /\left(\sqrt{2} \mu_{B}\right), \Delta_{0}$ being the zerotemperature energy gap in zero field and $\mu_{B}$ the Bohr 


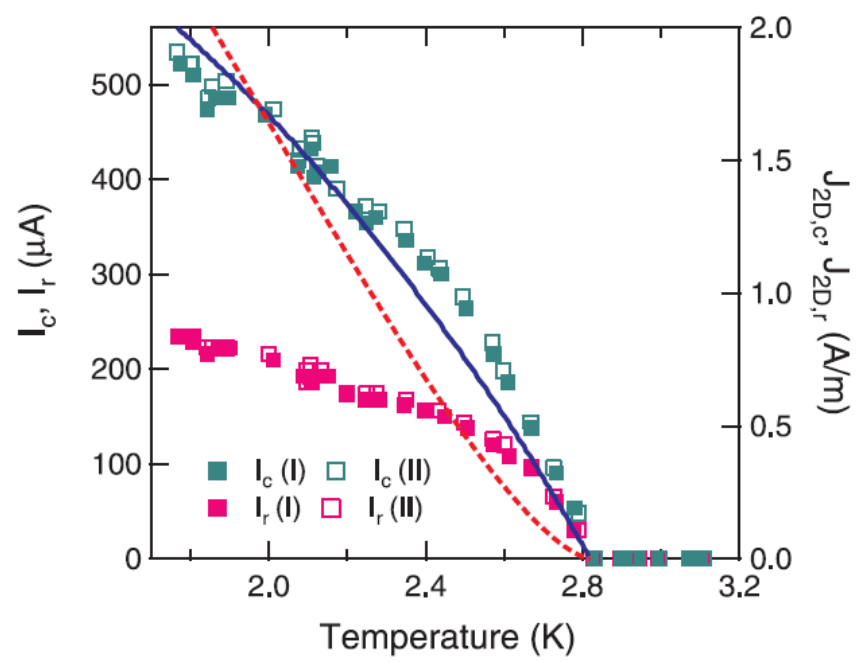

FIG. 13: Temperature dependence of the critical current of In monolayer grown on silicon surface measured in situ. In situ measured temperature dependences of the critical current $I_{c}$ (green squares) and retrapping current $I_{r}$ (pink squares) of the $\sqrt{7} \times \sqrt{3}-\operatorname{In} / \mathrm{Si}(111)$ grown in UHV. The data are measured with the configurations I and II, described in Fig. 12a, and are shown by closed and open squares respectively. The red dotted and blue solid lines show theoretical fits corresponding respectively to Cooper pair breaking and Josephson junction behavior. Note that there is a controversy regarding the actual In coverage of the $\sqrt{7} \times \sqrt{3}$ $\mathrm{In} / \mathrm{Si}(111)$ system, between a monolayer according to previous work ${ }^{8,99,102}$ and a double-layer according to recent DFT calculations ${ }^{103,104}$. Data are from Uchihashi et al. ${ }^{99}$.

magneton. For a weak-coupling BCS system one has $H_{\text {Pauli }}(T)=1.86 T_{c 0}(K)$, where $H_{P a u l i}$ is in Tesla and $T_{c 0}$ in Kelvin. Clearly this expression is not compatible with the effect reported in Fig. 14, since a critical field of about $2 \mathrm{~T}$ would be expected for the thinnest layer while almost no effect is seen up to $13.7 \mathrm{~T}$.

The most likely hypothesis consistent with the experimental data is that a mixed singlet-triplet order parameter would exist in the $\mathrm{Pb} / \mathrm{GaAs}(110)$ monolayer, as a consequence of a strong Rashba spin-orbit coupling ${ }^{117,118}$. Indeed a large Rashba spin-orbit coupling (SOC) ${ }^{119}$ is known to exist at the surface of heavy-element bulk metals, like $\mathrm{Au}(111)^{120}$, and has also been reported in various monolayers made out of $\mathrm{Bi}, \mathrm{Pb}$ or $\mathrm{Tl}$ heavy atoms grown on $\mathrm{Si}(111)$ or $\mathrm{Ge}(111)^{121-124}$. Let us note that very importantly, a large SOC has also been evidenced recently in a superconducting monolayer system : $(\mathrm{Tl}, \mathrm{Pb}) / \mathrm{Si}(111)^{108}$. The Rashba spin-splitting at $E_{F}$ measured by ARPES in these monolayer systems can be as large as $\Delta_{R}=100 \mathrm{meV}$, which is between two and three orders of magnitude larger than the reported superconducting energy gaps. One finds thus that the monolayers are in the following regime $T_{c} \ll \Delta_{R} \ll E_{F}$. As the SOC couples the electron's spin and momentum, the superconducting order parameter should develop a spin-triplet component, in addition to the $s$-wave

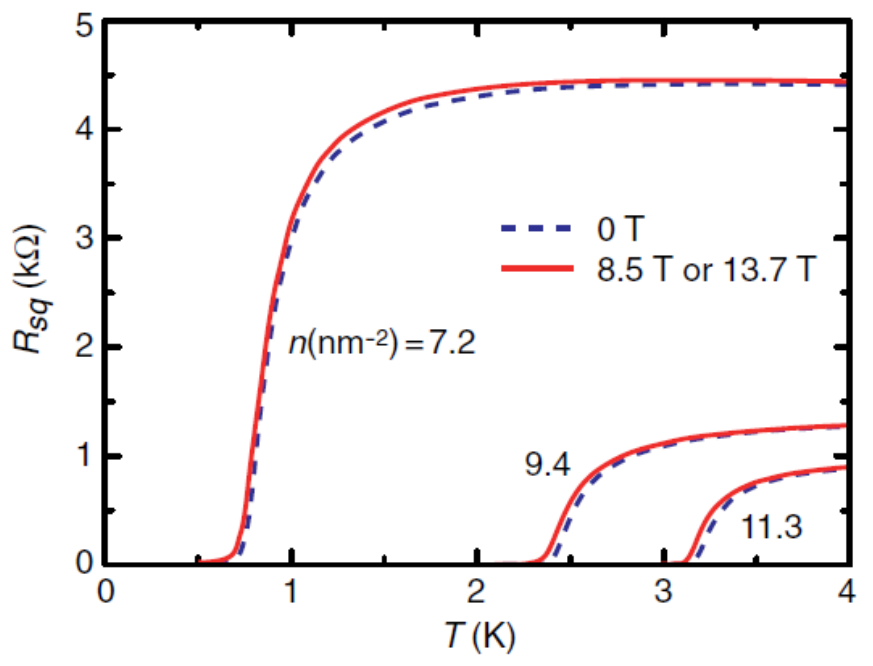

FIG. 14: In situ resistivity measurements in parallel magnetic field of $\mathrm{Pb}$ monolayers grown on GaAs. In situ measured temperature dependence of the square resistance of monolayer $\mathrm{Pb}$ films, grown on $\mathrm{GaAs}(110)$ under $\mathrm{UHV}$, for different atomic surface density of $\mathrm{Pb}$ atoms $n$ expressed in $\mathrm{nm}^{-2}$. Dashed curves are obtained for zero magnetic field. Solid curves are obtained in parallel magnetic field of $8.5 \mathrm{~T}$ (for $n=7.2 \mathrm{~nm}^{-2}$ ) or $13.7 \mathrm{~T}$ (for $n=9.4 \mathrm{~nm}^{-2}$ and $\left.11.3 \mathrm{~nm}^{-2}\right)$. Reproduced after Sekihara et al. ${ }^{114}$.

component ${ }^{117,118}$. As a result $H_{c}$, the critical magnetic field parallel to the surface, can be very high because an inhomogeneous superconducting state forms ${ }^{125}$. If the nonmagnetic disorder is not important, i.e. in the clean limit, the critical field should then be given by $H_{c}=\sqrt{\Delta_{0} \Delta_{R}} / \mu_{B}$, where $\Delta_{R}$ is the Rashba spin splitting at $E_{F}$. For $\Delta_{R}=0.1 \mathrm{eV}$ much larger than $T_{c 0}=1 \mathrm{~K}$ one gets $H_{c}=60 \mathrm{~T}$.

This inhomogeneous superconducting state is similar to the so-called Fulde-Ferell-Larkin-Ovchinnikov ${ }^{126,127}$ state but strongly modified by $\mathrm{SOC}^{125}$. If there is a high concentration of non-magnetic impurities the clean-limit picture can be changed ${ }^{128,129}$. According to the transport properties reported in monolayer superconductors by Sekihara et al. ${ }^{114}$, Uchihashi et al. ${ }^{99}$, Yamada et al. ${ }^{100}$ or Matetskiy et al. ${ }^{108}$, this condition seems to be always fulfilled: the dirty limit is reached, i.e. one has $\tau T_{c 0} / \hbar \ll 1$ where $\tau$ is the electron elastic scattering time. This latter condition is equivalent to the condition $l_{e} \ll \xi=\sqrt{\xi_{0} l_{e}}$, where $l_{e}$ is the elastic mean free path. Then for a Fermi energy $E_{F}$ larger than the Rashba spin splitting $\Delta_{R}$, which is most probably the situation encountered in all the studied superconducting monolayers up to now (as for example explicitely demonstrated by ARPES measurements in the $(\mathrm{Tl}, \mathrm{Pb}) / \mathrm{Si}(111)$ system $\left.^{108}\right)$, a weak helical state is predicted to survive up to a high critical field given by $H_{c}=\sqrt{\pi \hbar k_{B} T_{c 0} /\left(4 e^{\gamma} \tau\right)} / \mu_{B}$, which depends remarkably on $\tau$ but not on $\Delta_{R}$. If $\tau$ is estimated to be about 3 fs in ${ }^{114}$ from the normal state resistivity data, this leads to $H_{c}=48 \mathrm{~T}$, in agreement with the reported 
results.

\section{Berezinski-Kosterlitz-Thouless transition in monolayer superconducting films}

It was shown theoretically a long-time ago that at two dimensions, a system undergoing a phase transition to an order parameter with two real continuous components, such as the modulus and the phase of the superconducting order parameter given by $\Delta=|\Delta| e^{i \Phi}$, cannot exhibit true long-range order at $T>0^{130}$. It was further shown by Berezinskii, Kosterlitz and Thouless $(\mathrm{BKT})^{131,132}$ that in fact a quasi long-range order can be established below a critical temperature $T_{B K T}$ called the BKT temperature. Halperin, Nelson and Doniach, Huberman have further established that this situation also holds for 2D superconductors if their size is smaller than the Pearl screening length $L_{s}$ where $L_{s}=2 \lambda_{\perp}^{2} / d, \lambda_{\perp}$ being the bulk perpendicular London penetration length, $d$ the film thickness ${ }^{133,134}$. So in principle 2D superconducting and superfluid systems belong to the same universality class, the BKT one.

Here the 2D dimension refers to the order parameter dimensionality. For superconducting and superfluid thin films it only imposes that $\xi<d$, where $\xi$ is the coherence length and $d$ the film thickness. Systems relevant for BKT physics can in principle be much thicker than one atomic layer, the atomically thin limit corresponding to the situation we are mostly interested in in this review. Below $T_{B K T}<T_{B C S}$ vortex-antivortex pairs, which correspond to the thermal excitations of the superconductor or superfluid, can bind togoether through an attractive 2D Coulomb force.

A universal jump in the ratio of the superfluid density $n_{S}$ at $T_{B K T}$ divided by $T_{B K T}$ is predicted,

$n_{S} / k T_{B K T}=16 m /\left(\pi \hbar^{2}\right)(1)$.

While convincing experimental results have been observed in superfluid ${ }^{4} \mathrm{He}^{135,136}$, observations of the BKT regime in ultrathin superconducting films remained hotly debated ${ }^{137,138}$. The main difficulties to observe clear features of the BKT physics in superconducting ultrathin films are linked to the fact that :

i) the observations are restricted to a small temperature range $T_{B K T}<T<T_{B C S}$,

ii) BKT scenario requires ultraclean ultrathin samples so that effects produced by sample inhomogeneities and disorder, inducing inhomogeneous superconducting properties and vortex pinning, do not come into play.

Beasley et al. ${ }^{139}$ thought that using large squareresistivity disordered ultrathin films would help oberving BKT features. This is because from the relation (1) above one gets $T_{B K T}=\Phi_{0}^{2} / 32 \pi^{2} \times 1 / \lambda_{\perp}$, which leads in the dirty limit to $T_{B K T}=T_{B C S}\left(1-1.086 R_{\text {square }} / R_{K}\right)$, with $R_{K}=h / e^{2}=25.8 \mathrm{k} \Omega$. Thus the larger $R_{\text {square }}$ the lower will be $T_{B K T}$ with respect to $T_{B C S}$ and the larger will be the temperature interval to study BKT physics. However, the backdraw of using large resistive films is that the thermal energy required to create a vortex, which is of the order of the condensation energy $E_{c}$, is much reduced. As a consequence the probability $\exp \left(-E_{c} / k T_{B K T}\right)$ of creating vortices around $T_{B K T}$ is much increased with respect to superfluids. This should lead to a vortex density far too large to apply BKT theory which applies in a very dilute vortex density regime $^{140}$.

The other very problematic issue using large resistance films is that recent local STS experiments on homogeneously disordered films (see section IIB) clearly show that strong superconducting inhomogeneties develop in such systems ${ }^{39-43}$. As a consequence the unbinding of vortex-antivortex pairs above $T_{B K T}$, leading to a finite resistance value due to the displacement of free vortices, will be strongly influenced by the areas of the sample where lower energy gap values exist. Such areas will pin vortices not allowing them to move freely in the film, thus impeding the BKT physics to take place. Moreover, many sample areas could already in this regime have a closed energy gap value, thus definitely pinning vortices.

The regime which seems more favorable to observe BKT physics corresponds to low-disordered crystalline ultrathin films. A detailed study of $\mathrm{Hsu}$ and Kapitulnik performed on $2 \mathrm{~nm}$ thick single-crystal $\mathrm{Nb}$ films capped with $3 \mathrm{~nm}$ of amorphous Si addressed this issue ${ }^{107}$. The magneto-transport properties are measured ex situ. TEM measurements show that the Si capping does not affect the single-crystal structure of the $\mathrm{Nb}$ film. The film has $R_{\text {square }}=122 \Omega$. It is shown in Fig. 15a) that $R_{\text {square }}(T)$ can be analyzed in terms of the HalperinNelson formula to extract $T_{B K T}=3.60 \mathrm{~K}<T_{B C S}=$ $3.77 \mathrm{~K}$. The $T_{B C S}$ value found from this analysis was in agreement with Aslamasov-Larkin fluctuations analysis where an additional Maki-Thomson term has to be considered due to large phase coherence effects in this low-disordered crystalline film. Nevertheless, it can also be seen that the $R(T) / R_{n}$ curve can not be fitted down to the lowest square resistance values. There is a tail of low resistance values not captured by the BKT HalperinNelson formula. Hsu and Kapitulnik could show that in perpendicular magnetic field, this resistive tail is thermally activated and can be attributed to a flux creep regime of vortices. This flux creep regime occurs because vortex-antivortex pairs are pinned by the weak disorder present in the film, mostly attributed to thickness inhomogeneities, leading to a departure from the pure BKT regime.

BKT theory for superconducting films also predicts non linear $I-V$ characteristics for $T \leq T_{B K T}{ }^{133}$. In particular a non-linear dependence of the form $V \propto I^{\alpha(T)}$ is predicted, where $\alpha(T)>3$ for $T<T_{B K T}, \alpha(T)=3$ at $T=T_{B K T}$ and $1<\alpha(T)<3$ for $T_{B K T}<T<T_{B C S}$. 
a)

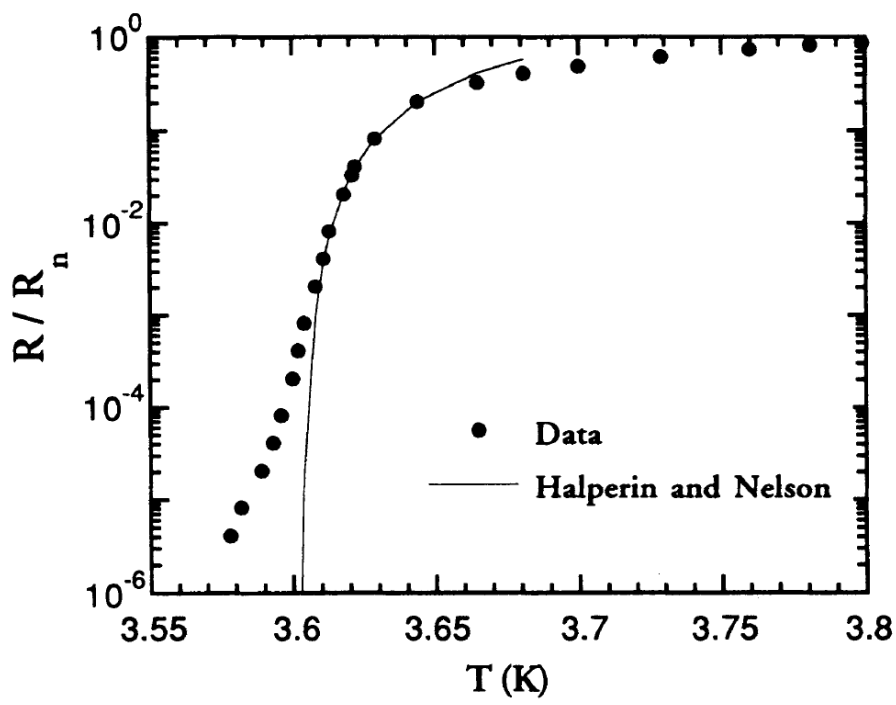

b)

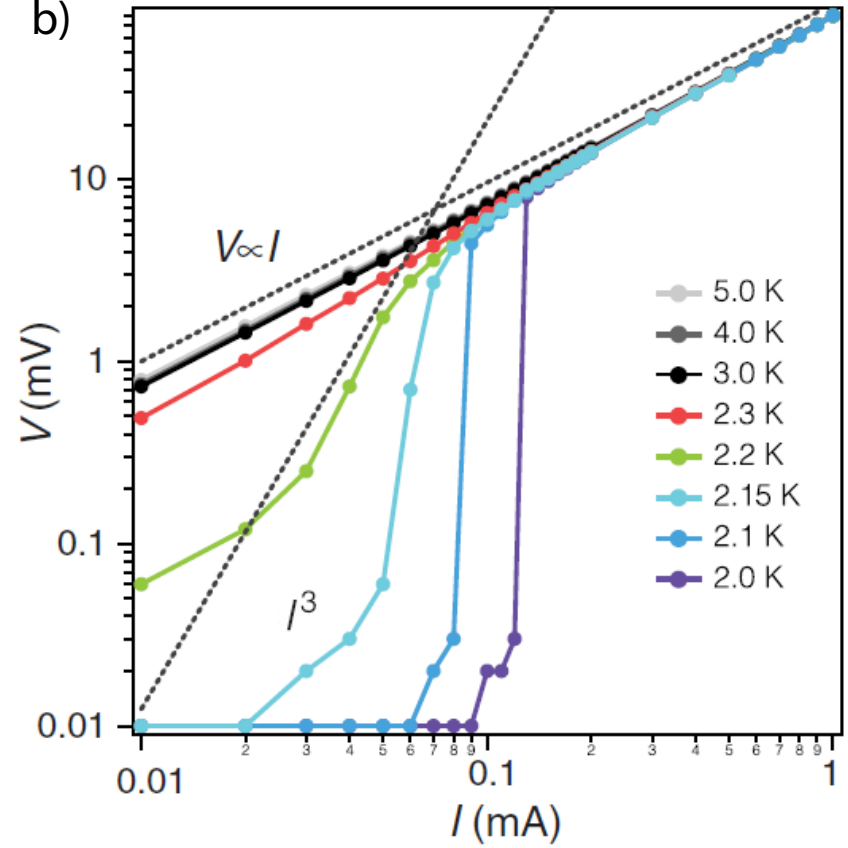

FIG. 15: Main features of BKT physics revealed in crystalline ultrathin and monolayer superconducting films from ex situ and in situ transport measurements. (a) ex situ measured square resistance $(R)$ normalized by its normal state resistance $\left(R_{n}\right)$ of a $2 \mathrm{~nm}$ thick single crystal $\mathrm{Nb}$ film capped with $3 \mathrm{~nm}$ of amorphous Si (logarithmic scale) versus temperature $T$ for zero applied field in a shielded Dewar. The solid line is a fit to the Halperin-Nelson BKT formula leading to $T_{B K T}=3.60 \mathrm{~K}$, and $T_{B C S}=3.77 \mathrm{~K}$. (b) in situ measured $I-V$ characteristics of a single atomic layer of thallium grown on $\mathrm{Si}(111)-7 \times 7$ under UHV, covered by $1 / 3$ of a $\mathrm{Pb}$ monolayer. The measurements are performed under zero magnetic field near the critical current for temperatures below $T_{B K T}$ to above $T_{B C S}$ and are plotted on a double-logarithmic scale. Two dashed lines indicate $V \propto I$ and $V \propto I^{3}$ curves. a) is reproduced from Hsu and Kapitulnik ${ }^{107}$ and b) from Matetskiy et al. ${ }^{108}$.

Such a dependence was not observed in Hsu and Kapitulnik experiment on crystalline $\mathrm{Nb}$ films ${ }^{107}$. On the other hand, such a power-law dependence with an exponent varying with temperature, could be demonstrated in several other low-disordered crystalline ultrathin films grown in UHV. In particular, it could be observed in 4 to $9 \mathrm{ML} \mathrm{Pb}$ films grown on $\mathrm{Si}(111)$ protected by a capping layer of $20 \mathrm{~nm}$ of amorphous $\mathrm{Si}^{141}$, in $2 \mathrm{ML} \mathrm{Ga}$ films grown on $\mathrm{GaN}(0001)$ capped with $80 \mathrm{~nm}$ of granular $\mathrm{Ag}^{142}$ and in a single atomic layer of $(\mathrm{Tl}, \mathrm{Pb})$ grown on $\mathrm{Si}(111)^{108}$. Among these three experiments, only the one of Matetskiy et al. was performed entirely in situ in UHV, i.e. growth and transport. In the other two cases, the films were grown in UHV but the transport experiments were performed ex situ, the films being protected with the help of a capping layer. The $V \propto I^{\alpha(T)}$ behavior close to $T_{B K T}$, taken from Matetskiy et al., is shown in Fig. 15b). The temperature $T=T_{B K T} \approx 2.2 \mathrm{~K}$, at which $\alpha(T)=3$, is in very good agreement with $T_{B K T}=2.24 \mathrm{~K}$ extracted from the analysis of the $R(T)$ dependence using the Haperin-Nelson formula.

In the $(\mathrm{Pb}, \mathrm{Tl})$ monolayer grown on $\mathrm{Si}(111)$ the superconducting energy gap and its temperature dependence could not be measured yet and be compared to $T_{B K T}$ and to the $R(T)$ behavior. Interestingly, such a comparison could be performed for the 4-9 ML Pb/Si(111) thin films of Zhao et al. Nevertheless, some care has to be taken for this comparison because as mentionned above, the transport measurements were performed on Si-capped films, while the gap measurements were peformed on bare $\mathrm{Pb}$ films grown in situ in UHV. Thus, as the capping is likely to increase disorder and reduce the available free electron density, it is expected that the energy gap of the bare surface should be larger than the one of the capped surface. The same behavior is thus expected for the corresponding $T_{B C S}$ of the two systems. Zhao et al found that below $9 \mathrm{ML}$ there is a separation between $T_{B K T}$ and $T_{\Delta}$, $T_{B K T}$ being defined and measured from ex situ transport as detailed above, and $T_{\Delta}$ being defined as the closing temperature of the gap measured in situ by STS. $T_{B K T}$ diminishes linearly with the number of $\mathrm{Pb}$ monolayer, while $T_{\Delta}$ remains at about its value for thicker films. Simultaneously the $T_{B C S}$ value, extracted from the analysis of the Aslamasov-Larkin fluctuations of the $R(T)$ dependence, is situated between $T_{B K T}$ and $T_{\Delta}$ for the thinnest films (4-6 ML) and merge together for thicker films (above $9 \mathrm{ML}$ ).

According to the BKT theory, $T_{B K T}$ is related to the $2 \mathrm{D}$ superfluid density $n_{S}^{2 D}\left(T_{B K T}\right)$ evaluated at $T=$ 
$T_{B K T}{ }^{143}$ by the following universal formula:

$k_{B} T_{B K T}=h^{2} n_{S}^{2 D}\left(T_{B K T}\right) / 16 \pi m k_{B}$,

$n_{S}^{2 D}(T)=n_{S}^{2 D}(0) \lambda^{2}(0) / \lambda^{2}(T)$ and $n_{S}^{2 D}(0)=d n_{S}^{3 D}(0)$,

where $d$ is the sample thickness, $n_{S}^{3 D}(0)$ the $3 \mathrm{D}$ superfluid density at $T=0$ and $m$ the electron mass. This universal formula captures well the linear dependence of $T_{B K T}$ with the film thickness $d$ observed by Zhao et $a l^{141}$, but fails quantitatively as a too large $T_{B K T}$ temperature is predicted if $n_{S}^{3 D}(0)$ is assumed to be equal to the $3 \mathrm{D}$ electron density in bulk $\mathrm{Pb}$. The consequence is that the real superfluid density realized in the superconducting thin films is reduced with respect to its expected clean BKT value by disorder effects ${ }^{25,144}$. A direct measurement of the superfluid density would be very helpful to better understand and model quantitatively the BKT physics at play in these crystalline ultrathin superconducting films.

Interestingly, BKT physics could be also demonstrated to be at play in other $2 \mathrm{D}$ systems like ultrathin high-temperature superconducting films ${ }^{145}$ and interface 2DEG in $\mathrm{LAO} / \mathrm{STO}^{6}$.

\section{LOCAL SUPERCONDUCTING} PROPERTIES FROM SCANNING TUNNELING SPECTROSCOPY MEASUREMENTS

According to in situ transport measurements reported in previous section $\mathrm{V}$ and ARPES measurements reported in section IV, the following conclusions can be drawn regarding the electronic properties of the monolayer phases $\mathrm{SIC}-\mathrm{Pb} / \mathrm{Si}(111), \sqrt{7} \times \sqrt{3}-\mathrm{Pb} / \mathrm{Si}(111)$ and $\sqrt{7} \times \sqrt{3}-\operatorname{In} / \mathrm{Si}(111)$. First, one can estimate the electronic elastic mean free path $l_{e}$ and the $k_{F} l_{e}$ product for the SIC phase. From the expression of the diffusion coefficient $D, D=v_{F} l_{e} / 2$ for a $2 \mathrm{D}$ metal $\left(v_{F}\right.$ being the Fermi velocity), one has at zero temperature $\xi(0)=\sqrt{\xi_{0} l_{e}}=$ $\sqrt{\hbar D / \Delta}$. We estimate $v_{F}$ from the $k_{F}$ and effective mass values quoted in section $\mathrm{IV}^{96}, v_{F}=\hbar k_{F} /\left(1.27 m_{e}\right)$ leading to $v_{F} \approx 10^{-6} \mathrm{~m} . \mathrm{s}^{-1}$. From transport measurements $\xi(0) \approx \xi_{G L} \approx 74 \mathrm{~nm}^{100}$, we get $l_{e} \approx 4 \mathrm{~nm}$ and $k_{F} l_{e} \approx 60$.

As $k_{F} l_{e} \gg 1$, this shows that the $\mathrm{Pb}$ SIC monolayer is very weakly disordered. In particular this system should be far from any emergent granular superconducting properties, which should appear when $k_{F} l_{e}$ becomes significantly smaller than 10 , as reported in section II B and observed by STS in the works ${ }^{39-43}$. These conclusions should also be true for the $\sqrt{7} \times \sqrt{3}-\mathrm{Pb} / \mathrm{Si}(111)$ and $\sqrt{7} \times \sqrt{3}$-In/Si(111) monolayers since their normal state square resistances before the superconducting transition are probably comparable $(\sqrt{7} \times \sqrt{3}-\mathrm{Pb}$ case $)$ or even smaller than the Pb-SIC one $(\sqrt{7} \times \sqrt{3}$-In case).

In conclusion, one expects from known effects of disorder in conventional superconducting ultrathin films that the local energy gap and the $d I / d V$ tunneling spectra measured by STS should remain almost constant all over
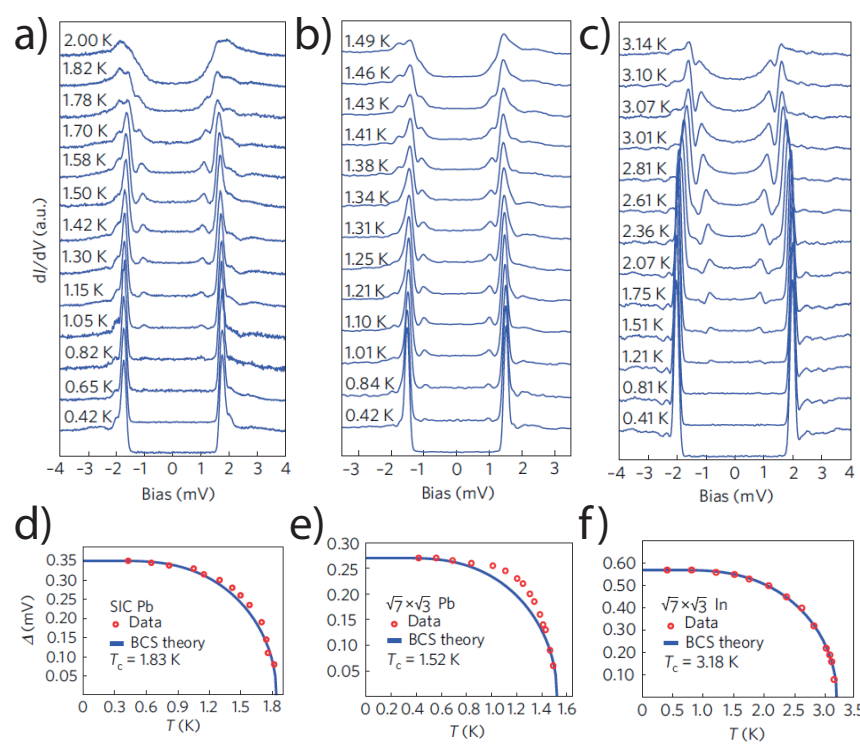

e)

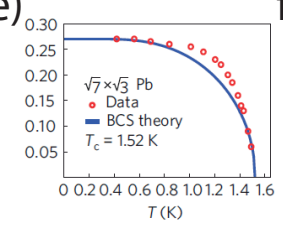

f)

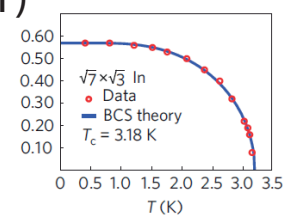

FIG. 16: Tunneling characteristics of $\mathbf{P b}$ and In supercondcuting monolayers grown on $\mathrm{Si}$ surfaces and measured in situ by STS. Differential conductance of singleparticle tunneling measured on three different monolayers by scanning tunneling spectroscopy with a $\mathrm{Nb}$ superconducting tip having an energy gap $\Delta=1.46 \mathrm{meV}$, for various temperatures. Tunneling set-point $V=10 \mathrm{mV}, I=0.2 \mathrm{nA}$. a) $\mathrm{SIC}-\mathrm{Pb} / \mathrm{Si}(111)$, b) $\sqrt{7} \times \sqrt{3}-\mathrm{Pb} / \mathrm{Si}(111)$ and c) $\sqrt{7} \times \sqrt{3}-$ $\mathrm{In} / \mathrm{Si}(111)$. The bottom panels d), e) ,f) show the temperature dependence of the energy gap extracted from BCS fits of the $d I / d V$ spectra. The actual In coverage of the $\sqrt{7} \times \sqrt{3}$ $\mathrm{In} / \mathrm{Si}(111)$ system was thought to be a monolayer according to previous works ${ }^{8,99,102}$ but may be a double-layer ${ }^{103,104}$. Reproduced after Zhang et al. ${ }^{8}$.

the surface. If there would exist superconducting inhomogeneities at $T \ll T_{c}$, these should be very small and characterized by very slight gap energy changes over length scales larger than the superconducting coherence length $\xi(0)$. We will see in the following three subsections that new superconducting properties emerge, not captured by current theories of disordered conventionnal 2D superconductors.

\section{A. New inhomogeneous superconducting} properties in monolayer superconductors induced by

2D electronic dimensionality : short-range spatial variations of the height of coherence peaks

Zhang et al. have reported conventional tunneling $d I / d V$ spectra measured by STS in the SIC-Pb, $\sqrt{7} \times \sqrt{3}-$ $\mathrm{Pb}$ and $\sqrt{7} \times \sqrt{3}$-In systems ${ }^{8}$. This is illustrated in Fig. 16 measured with a $\mathrm{Nb}$ superconducting tip having a gap of $1.46 \mathrm{meV}$ at $0.42 \mathrm{~K}$. The SIS tunneling spectra could be convincingly fitted assuming BCS DOS for the three MLs, BCS DOS for the Nb tip and a temperaturedependent $\Gamma$-Dynes broadening parameter $(\Gamma=6 \mu \mathrm{eV}$ at $0.42 \mathrm{~K})$. The extracted temperature dependent energy 
gaps are shown below each series of spectra. They do agree very well with BCS behavior for the SIC-Pb and $\sqrt{7} \times \sqrt{3}$-In systems, and show some deviation from BCS for the $\sqrt{7} \times \sqrt{3}$-Pb.

Brun et al. have reinvestigated by STS the electronic properties of the SIC-Pb and $\sqrt{7} \times \sqrt{3}-\mathrm{Pb} \mathrm{MLs}{ }^{112}$. We focus here first in this subsection on the results obtained on the SIC-Pb phase. The main new result is the observation of large spatial variations of the height of the coherence peaks (typically between 20 and $30 \%$ of relative change) over a length scale $l_{0}$ smaller than $10 \mathrm{~nm}$, while the superconducting enegy gap remains constant $^{112}$. This very surprising behavior is illustrated at the atomic scale in a $26 \times 26 \mathrm{~nm}^{2}$ area in Fig. $17 \mathrm{a}, \mathrm{b}, \mathrm{c}, \mathrm{d}$. A $2 \mathrm{D}$ s-wave weakly disordered superconductor should show almost constant energy gaps and peak heights as exposed above. On the other hand, Fig. 17 d reveals that the height of the coherence peaks in the SIC undergo large changes in this area, red (respectively blue) patches corresponding to red (blue) spectra in Fig. $17 \mathrm{c}$, on a length scale $l_{0}$ much smaller than the low-temperature SIC coherence length $\xi \approx 50 \mathrm{~nm}$ measured by STM through various effects $8,112,146$.

These peaks height changes are not directly crosscorrelated to the structural defects or $\sqrt{7} \times \sqrt{3}$ boundaries existing between the $\sqrt{3} \times \sqrt{3}$ domains seen in the topography (see Fig. 17a). It is important to note that in the experiment of Brun et al. shown in Fig. 17a,b,c,d, the coverage of the $\mathrm{SIC}-\mathrm{Pb}$ monolayer was in slight excess in purpose. The effective coverage was $1.40 \mathrm{ML}$ instead of 1.30 for the ideal SIC according to ${ }^{95}$. This additional amount of $\mathrm{Pb}$ atoms, about $0.1 \mathrm{ML}$ in excess deposited at room temperature, creates additionnal non-magnetic disorder which organizes in the form of adatoms and $1 \mathrm{ML}$ $\mathrm{Pb}$ islands grown on top of the SIC-Pb. As a consequence the energy gap measured by STS and extracted from the BCS fit of the red spectra in Fig. $17 \mathrm{c}$ is $\Delta=0.23 \mathrm{meV}$, which is smaller than $0.35 \mathrm{meV}$ reported by Zhang et al for the clean SIC-Pb having the ideal 1.30 ML coverage. Thus one could think that these additional non-magnetic disorder effects, inducing a smaller energy gap, could be fully responsible for these peaks height variations.

New experimental results performed by Brun et al. on the SIC-Pb ML, having a coverage much closer to the ideal 1.30 value, show that this is not the case and that the clean SIC behaves very much as reported previously $i^{112}$. The results are presented in Fig. 17e,f,g. The topography over a $300 \times 300 \mathrm{~nm}^{2}$ area, seen in panel e, shows indeed much less $\mathrm{Pb}$ adatoms and $1 \mathrm{ML}$ islands than in $^{112}$ (see Fig. 4a of this reference), while the size of the $\sqrt{3} \times \sqrt{3}$ areas is still comparable (between $5-10 \mathrm{~nm}$ ). In order to benefit from an increased energy resolution at $300 \mathrm{mK}$, STS measurements were performed with a superconducting PtIr tip coated with $\mathrm{Pb}$, having an energy gap about $1.30 \mathrm{meV}$. Thus the coherence peaks of the SIC-Pb appear now at $\pm\left(\Delta_{t i p}+\Delta_{S I C}\right)=$ $\pm 1.65 \mathrm{meV}$. This leads to an energy gap for the SIC of $0.35 \mathrm{meV}$ in very good agreement with the one reported by Zhang et al. ${ }^{8}$. Fig. $17 \mathrm{~g}$ shows the conductance map acquired on this $300 \times 300 \mathrm{~nm}^{2}$ area at the coherence peaks energy $\mathrm{eV}=1.65 \mathrm{meV}$ (similar maps are found for both energies). Large conductance variations are again observed, red and blue patches leading to the red and blue $d I / d V$ spectra presented in panel f. The increased energy resolution emphasizes a relative change of the peaks height of more than $30 \%$ between the red and blue regions. The size of constant peak height domains is consistent with the previous observation presented in panels a-d. The changes of the peaks height occur on a length scale $l_{0}$ much smaller than the SIC coherence length $\xi \approx 50 \mathrm{~nm}$, also consistent with the previous observation presented in panels a-d.

These low-temperature spatial variations of the height of the coherence peaks over a length $l_{0} \ll \xi$ are proposed by L. Ioffe and B. Altshuler in a toy model developped for a grain of size $L \gg l_{e} \gg \lambda_{F}{ }^{112}$, to be due to nonnegligible many-body electron-electron interaction terms produced by a BCS contact Hamiltonian ( $L$ is the grain size, $l_{e}$ the elastic electronic mean-free path and $\lambda_{F}$ the Fermi wavelength). These terms are non-negligible anymore because the electrons live in true two dimensions. These correcting terms add to the usual reduced BCS Hamiltonian, the latter being adapted when electrons live in a $3 \mathrm{D}$ space. These correcting terms, induced by the $2 \mathrm{D}$ dimensionality of the electron gas, lead to corrections in the quasiparticle excitation spectrum which reflect the variations of the local square conductance of the monolayer because the disorder has some spatial distribution and is not uniform. This happens although $G_{\text {square }} \gg G_{K}=2 e^{2} / h$, as $10<2 G_{\text {square }} / G_{K}<100^{100}$. In other terms, there is a link between a locally larger disorder and locally smaller and broader BCS coherence peaks and vice-versa. Theory predicts that this effect is accompanied by small energy gap changes. As experimentally such changes are not observed neither with a normal nor superconducting tip, it is inferred that these changes should be smaller than theoretically expected. Figure 18 shows that this toy-model theory enables reproducing almost quantitatively the experimental spectra shown in Fig. 17c, except for the small gap changes predicted for larger disorder. It also yields local $G$ values in good agreement with in situ transport measurements in the SIC-Pb of Yamada et al. ${ }^{100}$.

B. In-gap states induced by non-magnetic disorder in monolayer superconductors : hint for a mixed singlet-triplet superconducting order parameter induced by strong Rashba spin-orbit coupling

In this subsection we summarize the new results reported in the $\sqrt{7} \times \sqrt{3}-\mathrm{Pb} / \mathrm{Si}(111) \mathrm{ML}$ by Brun et al strongly suggesting the existence of a mixed singlettriplet order parameter ${ }^{112}$. 
a
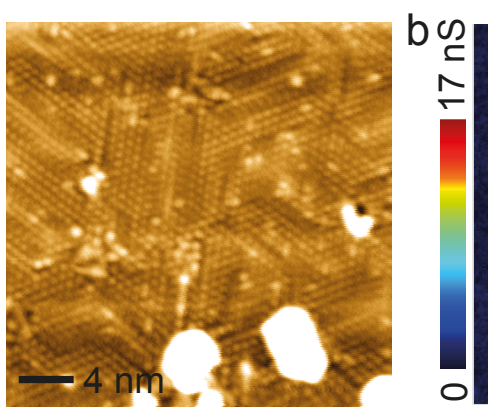

e

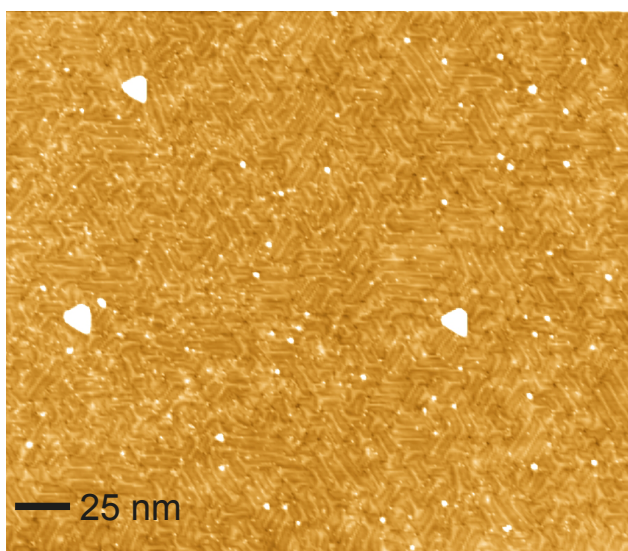

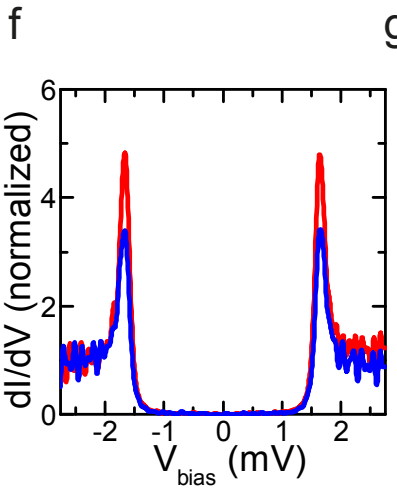
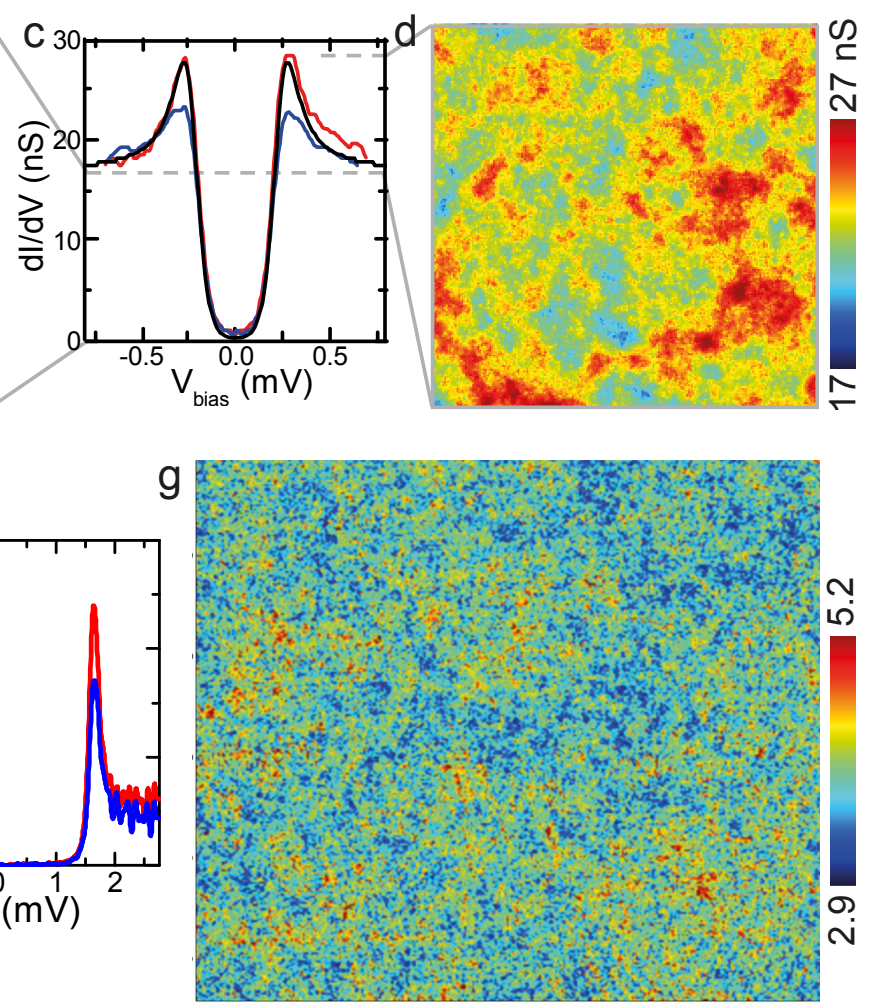

FIG. 17: Effect of 2D electronic dimensionality and weak non-magnetic disorder on the height of the coherence peaks of $\mathrm{Pb}$ superconducting monolayers. The local tunneling properties are measured at $T=300 \mathrm{mK}$ and in the top pannel with a normal PtIr tip while those in the bottom panel are measured with a superconducting tip enabling increased energy resolution (the tip energy gap is of $1.3 \mathrm{meV}$ ). (a),(e): Scanning tunneling microscopy images $(26 \mathrm{~nm} \times 26 \mathrm{~nm}, 290 \mathrm{~nm} \times 290 \mathrm{~nm}$ ) acquired on flat atomic terraces. Individual small $\sqrt{3} \times \sqrt{3}$ domains are visible (size from 5 to $10 \mathrm{~nm}$ ) separated by boundaries involving $\sqrt{7} \times \sqrt{3}$ periodicities. In addition various structural defects are seen : adatoms, vacancies, small $1 \mathrm{ML} \mathrm{Pb}$ islands ; (b): zero-bias conductance map measured on area a) or e) demonstrating that the superconducting energy gap exists everywhere on terraces and that no in-gap states are induced in the gap regardless of local or extended non-magnetic defects seen in (a) or (e). (c),(f): Representative local tunneling conductance spectra, showing that the amplitude of the quasiparticle coherence peaks strongly varies spatially while the gap remains constant. The red (respectively blue) curves were acquired in a few $\mathrm{nm}^{2}$ region with high (resp. low) coherence peaks, i.e. in red (blue) patches seen in (d),(g); the thin black solid line is a best fit of the red spectra using BCS formalism $\left(\Delta_{S I C}=0.23 \mathrm{meV}\right)$. No broadening parameter is needed for the red spectra. $(\mathrm{d}),(\mathrm{g})$ : Conductance maps at biases corresponding to the energy of the coherence peaks in (c),(f) (maps are similar for positive and negative peak energies). The spatial variations of the peaks amplitude do not follow directly the spatial distribution of local structural defects observed in the topographies; rather, there are several length scales involved. The smallest length yields a typical domain size of constant peaks height of few $\mathrm{nm}$. Spatial variations of peaks height occur on a length scale of less than $10 \mathrm{~nm}$, which is much smaller than the coherence length of the Pb-SIC which is of $50 \mathrm{~nm}$. At larger scale, the zones of constant peaks height appear filamentary and grainy, intertwined with lower peak height areas. Tunneling set-point for spectroscopy $V=5 \mathrm{mV}, I=0.2 \mathrm{nA}$. Top panels are adapted from Brun et al. ${ }^{112}$, while bottom ones are new data from the authors presented in this review paper.

In the Pb-SIC ML Brun et al reported the existence of new short-ranged coherence peaks height variations. However, no in-gap quasiparticle states were observed for this system. This was illustrated by the homogeneous conductance map shown in Fig. 17b measured at $0 \mathrm{meV}$ energy. In the Pb-SIC, any other energy inside the superconducting gap would show the same homogeneous conductance map equal to zero.

A very different behaviour is observed in the $\sqrt{7} \times \sqrt{3}$ $\mathrm{Pb} / \mathrm{Si}(111) \mathrm{ML}$. First, the tunneling conductance spectra at any location depart from BCS behavior as shown in Fig. 19c. The coherence peaks are everywhere much lower and broader than pure BCS theory would predict. Furthermore, gap filling is everywhere remarkable. Moreover, this gap filling fluctuates over a length scale of about $10 \mathrm{~nm}$ which is much shorter than $\xi_{\sqrt{7} \times \sqrt{3}} \approx$ $45 \mathrm{~nm}$. This is illustrated in Fig. 19b. Simultaneously the height of the coherence peaks spatially varies, again on a length scale smaller than $\xi_{\sqrt{7} \times \sqrt{3}} \approx 45 \mathrm{~nm}$ (Fig. 19d). The zero-bias and peaks energy conductance maps are not directly correlated to each other. Moreover these two conductance maps do not follow directly the local defects 


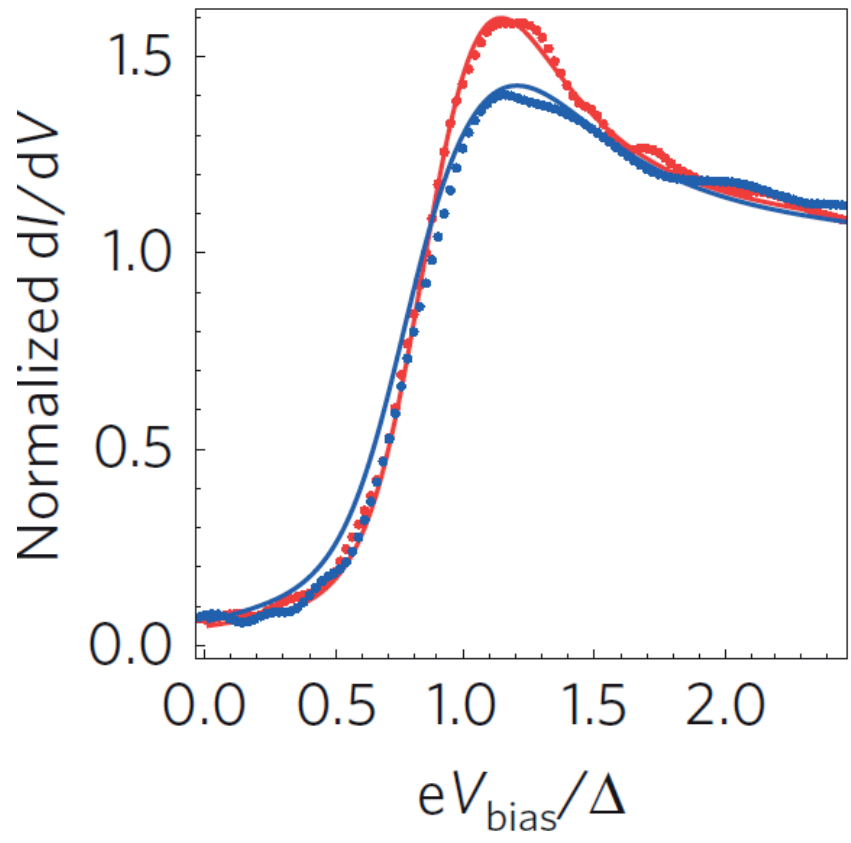

FIG. 18: Theoretical modelling of the spatial variations of the height of the coherence peaks induced by 2D superconductivity. Fit of the experimental data shown in Fig. 17c (dots) to the expected $d I / d V$ spectra that takes into account non-zero temperature, small dephasing rate and small gap variations. The red and blue continuous curves are the theoretical expectations for different values of the parameter $g=0.01$ and $g=0.03$ respectively, where $g \approx 50 / G^{2}$, $G$ being the local dimensionless square conductance value (in $e^{2} / h$ units). This yields $G=71$ and 41 respectively, in good agreement with in situ transport data of Yamada et $a l^{100}$. The best fit is obtained if one assumes that the bare gap value is slightly larger $\Delta_{g=0.03}=1.05 \Delta_{g=0.01}$ for the more disordered location. In all curves $T=0.1 \Delta$ and $\Gamma=\hbar / \tau_{\phi}=0.05 \Delta$. The quality of the fit is only weakly sensitive to the actual values of $T$ and $\Gamma$ : one can produce very similar fits assuming slightly higher $T$ and lower $\Gamma$. Reproduced after Brun et al. ${ }^{112}$

or twin boundaries observed in the topography Fig. 19a.

In order to rationalize these findings one first needs to remember the well-known effects of non-magnetic disorder on ultrathin superconducting films reported in section II. In particular for weakly disordered films, nonmagnetic disorder cannot induce in-gap states for $s$-wave superconductors. Thus if $\sqrt{7} \times \sqrt{3} / \operatorname{Si}(111)$ would be a conventional $s$-wave superconductor, the only theoretical possibility consistent with the experimental results would be that the in-gap states should be induced by magnetic impurities. However the high purity of the $\mathrm{Pb}$ source and Si substrate used in the experiment of Brun et al enables to exclude such a possibility, as this would lead to a too high density of magnetic impurities. Moreover, as the same $\mathrm{Pb}$ source and $\mathrm{Si}$ substrate were used to grow the SIC-Pb ML, magnetic impurities signatures should have also been observed in the superconducting gap of the SIC-Pb ML, in contradiction with the experimental results reported in subsection VIB. We thus conclude that the $\sqrt{7} \times \sqrt{3}-\mathrm{Pb} M L$ is not a pure s-wave superconductor.

The simplest assumption in agreement with the experimental results is that, following Rashba and Gorkov, the $\sqrt{7} \times \sqrt{3}$-Pb ML possess a $p$-wave superconducting component, i.e. this system has a mixed singlettriplet order parameter induced by a strong spin-orbit coupling ${ }^{117,118}$. Indeed as already reported in section V C, a strong Rashba spin-orbit coupling should exist in the $\sqrt{7} \times \sqrt{3}$-Pb ML, as recently investigated by DFT calculations for the dense $\sqrt{3} \times \sqrt{3}-\mathrm{Pb} / \mathrm{Si}(111)$ and $\mathrm{Ge}(111)$ phase $^{93}$. A large Rashba spin-orbit coupling (SOC) ${ }^{119}$ is known to exist at the surface of heavy-element bulk metals, like $\mathrm{Au}(111)^{120}$, and has also been reported in various monolayers made out of $\mathrm{Bi}, \mathrm{Pb}$ or $\mathrm{Tl}$ heavy atoms grown on $\mathrm{Si}(111)$ or $\mathrm{Ge}(111)^{121-124}$. Very importantly, a large SOC has also been evidenced recently in a superconducting monolayer system : $(\mathrm{Tl}, \mathrm{Pb}) / \mathrm{Si}(111)^{108}$.

The Rashba spin-splitting at $E_{F}$ measured by ARPES in these monolayer systems can be as large as $\Delta_{R}=$ $100 \mathrm{meV}$, which is between two and three orders of magnitude larger than the reported superconducting energy gap $\Delta$ of $0.2-0.3 \mathrm{meV}$. One thus finds that the $\sqrt{7} \times \sqrt{3}-\mathrm{Pb}$ ML presents the following energy scales $T_{c} \ll \Delta_{R} \ll E_{F}$. The assumption of a $p$-wave superconductor in $\sqrt{7} \times \sqrt{3}$ $\mathrm{Pb} \mathrm{ML}$ is also supported by the insensibility of in situ transport measurements to high parallel magnetic field in a $\mathrm{Pb}$ ML on GaAs(110), going much above the Pauli paramagnetic limit ${ }^{114}$.

The way quasiparticle states would be induced inside the $\sqrt{7} \times \sqrt{3}-\mathrm{Pb}$ superconducting gap is now simple to understand. Because of a large spin-triplet component of the order parameter, non-magnetic defects behave in the very same way magnetic impurities are known to form $\mathrm{Yu}$-Shiba-Rusinov bound states inside the gap of an $s$ wave superconductor ${ }^{147-155}$. Such a sensibility of $p$-wave superconductors to non-magnetic disorder was studied in details for example in $\mathrm{Sr}_{2} \mathrm{RuO}_{4}{ }^{156,157}$. We believe that a similar situation occurs in the $\sqrt{7} \times \sqrt{3}$ - $\mathrm{Pb}$ ML although the $p$-wave component is of different nature in both systems.

Further ARPES experiments are needed to study the precise spin-splitting of the electron bands in the $\sqrt{7} \times$ $\sqrt{3}-\mathrm{Pb}$ system in order to enable a theoretical modelling of the $p$-wave superconductivity at play in this ML. This should enable further theoretical and experimental work addressing the effect of both nonmagnetic and magnetic impurities in this Rashba 2D superconductor. Further experimental and theoretical work is also needed to study whether the $\sqrt{7} \times \sqrt{3}$-In system also presents $p$-wave superconductivity. Finally, it is not clear at present why the $\mathrm{SIC}-\mathrm{Pb}$ monolayer does not present such a sensitivity as the $\sqrt{7} \times \sqrt{3}$-Pb to non-magnetic disorder, while it most probably also undergoes a large Rashba spin-orbit coupling effect ${ }^{93}$. We speculate that the $\mathrm{SIC}-\mathrm{Pb}$ should also present a $p$-wave component. However for some yet unknown reasons, the scattering effects on the $p$-wave 

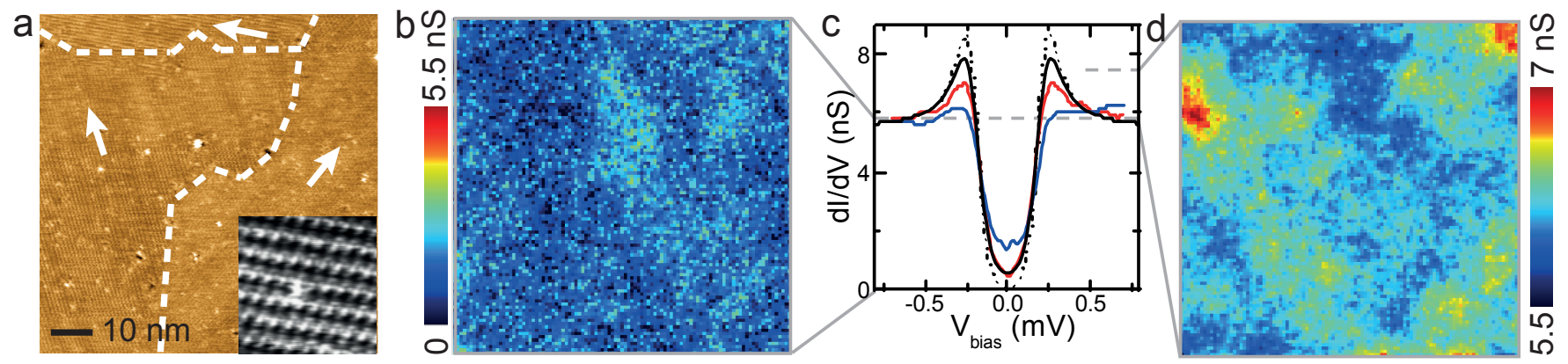

FIG. 19: In-gap states induced by non-magnetic disorder in $\mathbf{P b}$ superconducting monolayers : hint for a mixed singlet-triplet superconducting order parameter induced by strong Rashba spin-orbit coupling. The local tunneling properties are measured at $T=300 \mathrm{mK}$ with a normal PtIr tip. (a): Scanning tunneling microscopy images $(85 \mathrm{~nm} \times 85 \mathrm{~nm})$ acquired on flat atomic terraces. Various structural defects are seen : adatoms, vacancies, voids, small $\mathrm{Pb}$ clusters; the dashed lines delimit three different rotational domains indicated by arrows. The inset shows the unit cell resolution. (b): zero-bias conductance map measured on area a) demonstrating that in-gap states are induced inside the superconducting gap by the non-magnetic disorder seen in topography a). This gap filling undergoes large spatial variations on length scale much smaller than the superconducting coherence length $\xi \approx 45 \mathrm{~nm}$. (c): Representative local tunneling conductance spectra showing different gap filling. The red (respectively blue) curves were acquired in a few $\mathrm{nm}^{2}$ region with lower (resp. higher) zero-bias conductance, i.e. in darker (lighter) patches seen in (b); the thin dotted black line is a best fit of the red spectra using BCS formalism and no broadening $(\Delta=0.20 \mathrm{meV})$. The thick continuous black line is a best BCS fit of the red curve using a $\Gamma$-Dynes broadening parameter $(\Gamma=0.02 \mathrm{meV})$. (d): Conductance map at bias corresponding to the energy of the coherence peaks $0.26 \mathrm{meV}$ in (c) (maps are comparable for both peak energies). Similar to the SIC-Pb case, spatial variations of the peaks amplitude are observed on length scale much smaller than $\xi$. Both zero-bias (in b) and peaks height variations (in d) do not follow directly the distribution of local structural defects or twin boundaries observed in the topography; adapted from Brun et al. ${ }^{112}$.

component that should be produced by non-magnetic disorder average to zero. The reasons for this important difference with respect to the behavior of the $\sqrt{7} \times \sqrt{3}-\mathrm{Pb}$ might be related to the absence of a true commensurate periodic potential in the $\mathrm{SIC}-\mathrm{Pb}$. Interestingly, spectroscopic signatures of Rashba spin-split states, that have been measured by ARPES in the related $\mathrm{Pb} / \mathrm{Ge}(111)$ $\beta \sqrt{3} \times \sqrt{3}$ monolayer, were searched for by STS using proximity effects to $\mathrm{Pb}$ islands ${ }^{158}$. However Kim et al. could not evidence any peculiar proximity effect that would be induced by a Rashba spin-orbit coupling in a two-dimensional superconductor ${ }^{159,160}$. Moreover in contrast to the SIC-Pb there seems to be a large contribution from subsurface electron states in the $\mathrm{Pb} / \mathrm{Ge}(111)$ $\beta \sqrt{3} \times \sqrt{3}$ system.

\section{Vortex properties and role of step edges in monolayer superconducting films}

The study of vortices in the presence of magnetic field is particularly important as it may enable revealing important characteristics of the superconducting condensate such as anistropy, a multiband or multigap character of the system, or unconventional superconductivity. Studies of Zhang et $a l^{8}$, Brun et $a l^{112}$ and Yoshizawa et $a l^{113}$ addressed this issue by studying $\mathrm{Pb}$ and In monolayers on $\mathrm{Si}(111)$ by STM/STS under perpendicular magnetic field. A very different behaviour was reported between the one of the SIC-Pb ML on one hand and the one of the $\sqrt{7} \times \sqrt{3}$-Pb and $\sqrt{7} \times \sqrt{3}$-In on the other hand. The main finding is that atomic steps connect well the superconducting condensate in the case of the SIC-Pb $\mathrm{ML}^{112}$ while atomic steps behave as Josephson junctions in the case of the $\sqrt{7} \times \sqrt{3}-\mathrm{Pb}^{112}$ and $\sqrt{7} \times \sqrt{3}-\operatorname{In}^{113}$. The local STS results in the $\sqrt{7} \times \sqrt{3}$ systems nicely confirms the hypothesis inferred from macroscopic in situ transport measurements of Uchihashi et al in $\sqrt{7} \times \sqrt{3}-\operatorname{In}^{99}$.

\section{1. zero magnetic field properties at step edges}

The fact that in SIC-Pb atomic steps are highly transparent for Cooper pairs, while this is not the case in $\sqrt{7} \times \sqrt{3}-\mathrm{Pb}$ and $\mathrm{In}$, leads to noticeable differences already in zero-field. In $\mathrm{SIC}-\mathrm{Pb}$ the tunneling conductance spectra and the gap do not change across a step edge, which is illustrated in Fig. 20a,b. Nevertheless, monoatomic steps of the $\mathrm{SIC}-\mathrm{Pb}$ are significant sources of non-magnetic scattering, as shown by a study of the proximity effect between a superconducting $\mathrm{Pb}$ nanoisland and the SIC-Pb in its normal state at $2.15 \mathrm{~K}^{161}$. In principle such an S-N proximity effect should be treated self-consistently, as in the similar study of Cherkez et al. ${ }^{146}$, because true superconductivity is induced in the $\mathrm{SIC}$ in the vicinity of $\mathrm{Pb}$ islands. However, Kim et al. assumed zero order parameter in the SIC-Pb, as in another former study ${ }^{162}$, and extracted that about half the square resistance of the SIC is due to scattering by the surface atomic steps ${ }^{161}$. 
On the other hand inhomogeneous superconducting properties are often observed in $\sqrt{7} \times \sqrt{3}-\mathrm{Pb}$ close to step edges as shown in Fig. 20c,d. Simultaneously, the atomic structure measured by STM in the topography of these regions present the same $\sqrt{7} \times \sqrt{3}$ - $\mathrm{Pb}$ characteristics as those measured in the terrace interior ${ }^{112}$. It was found that in such areas, called nano-protrusions (of sizes from few nm up to $30 \mathrm{~nm}$ ), attached to the upper terrace (the nano-protrusions are the sample areas delimited by the white dashed line in Fig. 20c), superconductivity is weakened as shown by the zero-bias conductance map on Fig. 20d.

The spatial evolution of the spectra measured on the upper terrace across a nano-protrusion (along the white dashed line seen in Fig. 20d) reveals in Fig. 20e that proximity effect takes place between the terrace interior and the nano-protrusion ${ }^{146,163}$. Thus the nanoprotrusions could be intrinsically either non-superconducting or having a much smaller energy gap than in the $\sqrt{7} \times \sqrt{3}$ $\mathrm{Pb}$ interior. Brun et al have proposed that there could be a mixture of $\mathrm{Pb}$ and $\mathrm{Si}$ atoms in these areas, weakening superconductivity. This is however not settled yet and should be further studied. Another possibility could be that this weakening could be related to the extreme sensitivity of the $\sqrt{7} \times \sqrt{3}$ - $\mathrm{Pb}$ monolayer to non magnetic disorder, known to induce subgap states ${ }^{112}$, as reported above in section VIB. In this case, as there is indeed increased scattering close to step edges, this could help rationalize the observed behavior.

A spectacular abrupt change of the tunneling spectra is observed at the step edge crossing between the upper and lower terrace, shown in $20 \mathrm{e}$ and seen beautifully in the zero-bias conductance map in $20 \mathrm{~d}$. The nano-protrusions enable revealing that step edges act as Josephson barriers for Cooper pairs with a low transparency ${ }^{146,163,164}$.

\section{2. magnetic field properties at step edges}

In $\mathrm{SIC}-\mathrm{Pb}$ the atomic steps are highly transparent for Cooper pairs. They have no noticeable effect on the vortex shape that is round and on their organization that forms a disordered triangular lattice, as shown in Fig. 21a. Thus these vortices are fully Abrikosov-like, except for the extreme type II behavior of these ultrathin superconductors for which they should be called Pearl vortices $^{83,85,165}$. For simplicity we will continue to name them Abrikosov vortices in the following. The superconducting coherence length can be extracted from the zero-bias conductance profile through an isolated vortex, leading to $\xi_{S I C} \approx 50 \mathrm{~nm}^{8,112}$.

On the contrary, the fact that the $\sqrt{7} \times \sqrt{3}-\mathrm{Pb}$ monolayer is a network of native Josephson junctions strongly influences the vortex phase. At low applied magnetic field all vortices are located along atomic steps (see Fig. 21b); their cores have various sizes and shapes. Some vortices appear elongated along the steps but shrank in the perpendicular direction. Others seem to almost disappear having a very shallow aspect. This phenomenon is not limited to $\mathrm{Pb}$ : Yoshizawa et $a l^{113}$ observed a very similar phenomenon in $\sqrt{7} \times \sqrt{3}$-In (see Fig. $21 \mathrm{~d}$ in $0.04 \mathrm{~T}$ and Fig. 21c measured in a remnant field).

The reason why the vortices prefer atomic step edges is clear: Since the energy cost of a vortex core is proportional to the superconducting order parameter, this cost is significantly lower in locations where the superconductivity is weakened, i.e. at step edges in the case of the $\sqrt{7} \times \sqrt{3}-\mathrm{Pb}(\mathrm{In})$-monolayers.

The deformation of the vortex cores is a more complex question; it is related to the fact that the characteristic energy of the junction is the Josephson energy

$E_{J}=\frac{\hbar j_{c}^{s t e p}}{2 e}$

where $j_{c}^{\text {step }}$ is the maximum Josephson supercurrent density (per unit of length for a $2 \mathrm{D}$ system) that the junction can support. At the low temperature of the experiment, $j_{c}^{\text {step }}(r)$ is given by the AmbegaokarBaratoff relation

$j_{c}^{s t e p}(r)=\frac{\pi \Delta(r)}{2 e \rho_{\text {step }}}$,

where $\rho_{\text {step }}$ is the electric resistivity of the step in the normal state (in $\Omega . \mathrm{m}$ ). The density $j_{c}^{\text {step }}$ is lower than the critical supercurrent density $j_{c}$ in the superconducting terrace, $j_{c}^{\text {step }} \leq j_{c}$. Therefore, by current density conservation the density of currents circulating around a vortex pinned at a step edge is limited by $j_{c}^{\text {step}}$; it is lower than the density of currents circulating around a conventional Abrikosov vortex located in a terrace. As a consequence, the current loops become elongated along the step edges, see left panels in Fig. 21f.

The current crossing the step edge flows owing to a phase difference, obeying the Josephson relation

$j^{\text {step }}(r)=j_{c}^{\text {step }}(r) \sin (\Delta \phi(r))$

where $\Delta \phi(r)$ is the gauge-independent phase difference between two points situated at opposite sides of the step edge at a location $r$. Therefore, in order to have current flowing, the system has to create two phase drops $\Delta \phi^{\text {step }}$ across the Josephson barrier B drawn in the middle panels of Fig. 21f, between points 1-4 and $2-3$. Now, let us consider the vortex singularity located inside the barrier. The vortex currents are given by the following expression:

$$
\vec{j}(r)=\frac{2 e \hbar}{m^{*}}|\Psi(r)|^{2} \vec{\nabla} \phi(r)
$$

They make loops (indicated by dashed lines) owing to the phase gradients. The total phase accumulation over the loop is obviously $2 \pi$. Due to the two phase drops $\Delta \phi^{\text {step }}$ in the barrier $\mathrm{B}$, each phase accumulation on each terrace (between points 1 and 2, and between 3 and 4) is less than $\pi$, 
$\Delta \phi_{12}=\Delta \phi_{34}=\pi-\Delta \phi^{\text {step }}<\pi$,

while it is exactly $\Delta \phi_{12}=\Delta \phi_{34}=\pi$

in the case of an Abrikosov vortex. Thus, the phase gradients around the paths 12 (and 34) are lower in comparison to the case of an Abrikosov vortex, resulting in a lower superfluid velocity $\overrightarrow{v_{S}}$. This reduced superfluid velocity provokes a weaker depairing effect on Cooper pairs than in the Abrikosov vortex case. Consequently, the suppression of the order parameter is weaker. Near the vortex centre one has:

$\mid \Psi(r \rightarrow$ center $) \mid) \approx\left[1-\left(\frac{j_{c}^{\text {step }}}{j_{c}}\right)^{2}\right]|\Psi(\infty)|$

where $\Psi(\infty)$ is the unperturbed order parameter in zero magnetic field and zero currents ${ }^{113}$.

Remarkably, the order parameter does not become zero as in the case of an Abrikosov vortex, but remains finite in presence of a Josephson barrier (right panels in Fig. 21f). This latter expression allows one to distinguish three characteristic cases that this formula links together: pure Abrikosov vortex, pure Josephson vortex and intermediate mixed Josephson-Abrikosov case ${ }^{166}$.

Indeed, when the barrier is highly transparent, $j_{c}^{\text {step }}=$ $j_{c}$, one obtains the full suppression of the order parameter in the core centre. This is the Abrikosov case. In the opposite limit, when the Josephson link is very weak: $j_{c}^{\text {step }} \ll j_{c}$ and this results in $\mid \Psi(r \rightarrow$ center $)|\approx| \Psi(\infty) \mid$. In this case, the superconducting order parameter around the phase singularity remains unaffected. The intermediate case of a mixed Abrikosov-Josephson vortex is realized in the $\sqrt{7} \times \sqrt{3}$-Pb and In-monolayers in Fig. 21b,c,d showing distorted vortex cores elongated along the steps and having a smaller amplitude than Abrikosov vortices shown in Fig. 21a,e,d. These observations are supported theoretically by microscopic Bogoliubov-deGennes numerical calculations in a $2 \mathrm{D}$ tight-binding model ${ }^{113}$.

Unfortunately, when approaching a pure Josephson situation where $\mid \Psi(r \rightarrow$ center $)|\approx| \Psi(\infty) \mid$, the spatial variations of $\Psi(r)$ become less and less pronounced, and the spectral signal of the vortex in STS maps vanishes (for instance compare the difference in zero-bias conductances between the mixed vortices A' and B' in Fig. 21c). Nevertheless, the study of pure Josephson vortices by STS is still possible in SNS proximity junctions ${ }^{167}$.

In rising magnetic field in $\sqrt{7} \times \sqrt{3}$ - $\mathrm{Pb}$ and $\mathrm{In}$, more and more mixed Abrikosov-Josephson vortices located and elongated along the step edges appear; the intervortex distance decreases. At some field the steps get saturated by vortices; the energy cost of placing an additional vortex core at the step edge becomes higher than the energy required to create a usual Abrikosov vortex in the nearby terraces. That is why, at higher magnetic field the new vortices appear in terraces, as demonstrated in Fig. 21e,d. In Fig. 21e only the new vortices that appeared between $0.04 \mathrm{~T}$ and $0.08 \mathrm{~T}$ are shown. This map clearly demonstrates that none of them is located at a step.

Notice that the spatially inhomogeneous superconductivity and possibly the high mobility of vortices in the terraces of the $\sqrt{7} \times \sqrt{3}$-Pb monolayer could explain the observed irregular shapes of the Abrikosov vortex cores in Fig. 21e. In the Indium system, the more regular round shape of the Abrikosov vortex cores could also be due to the actual thickness being in fact 2 monolayers instead of one, creating a larger core energy.

\section{CONCLUSION}

We have presented in this review the recent advances performed in the last ten years in the field of ultrathin superconductors made of a single atomic plane of simple metal elements like $\mathrm{Pb}$ or In grown on a semiconducting substrate like $\mathrm{Si}(111)$, using molecular beam epitaxy methods. These systems constitute perfect $2 \mathrm{D}$ superconductors where, in addition, the electrons are truly twodimensional, as envisionned long ago by V. Ginzburg. These systems are extremely well-controlled structurally, as they are made of a crystalline atomic monolayer lying directly at the surface: they are accessible to every surface physics characterization tools like LEED, RHEED, XPS, SXRD. They are also accessible to state-of-theart electronic spectroscopic tools like ARPES, STS and in situ magneto-electrical transport. As a consequence, they offer a full control of the crystalline quality of the atomic structure to be confronted in full details to ab initio DFT calculations enabling to describe quantitatively both structural and electronic properties.

This favorable situation leads to a rather good understanding of their structural and electronic properties. However an important aspect of these systems is being only now taken into account in DFT calculations: the strong-spin orbit coupling (SOC). This step is crucial from several point-of-views, and in particular in order to fully model and understand the superconducting properties beyond the conventional behavior reported initially ${ }^{8}$ :

i) It appears that the ground-state of the crystal is modified when taking into account spin-orbit coupling, giving a better agreement with the structures found from experimental structural studies ${ }^{93}$.

ii) STS measurements of the local superconducting properties reveal that in-gap states are induced in the superconducting gap of the $\sqrt{7} \times \sqrt{3}$ - $\mathrm{Pb}$ monolayer by non-magnetic disorder ${ }^{112}$. This implies that a mixed singlet-triplet superconducting order parameter exist in these systems induced by a strong Rashba spin-orbit coupling ${ }^{11-119}$. From STS and ARPES measurements one encounters the following regime: $\Delta \ll \Delta_{R} \ll E_{F}$, $\Delta$ being the superconducting gap, $\Delta_{R}$ the spin-orbit energy splitting at $E_{F}, E_{F}$ being the Fermi energy of the monolayer. In situ transport measurements in high parallel magnetic field in a $\mathrm{Pb} / \mathrm{GaAs}(110)$ monolayer 
are consistent with these results ${ }^{114}$ and a similar physics should exist in related superconducting monolayers where a large SOC was measured by ARPES ${ }^{108}$. A similar behavior may also take place in thicker crystalline films as recent experiments on capped $\mathrm{Pb} / \mathrm{Si}(111)$ films suggest $^{168}$.

iii) Several other important achievements were reported enlightening unexpected new properties of superconducting systems made of 2D-electrons. Namely, spatial variations of the coherence peaks height over length scales much smaller than the superconducting coherence length were observed and linked theoretically to local variations of the nonmagnetic disorder ${ }^{112}$. A peculiar role of monoatomic steps behaving as Josephson junctions with low-transparency was found in monolayer superconductors with an anisotropic bandstructure ${ }^{99,112,113}$. This led to the observation of mixed Abrikosov(Pearl)-Josephson anisotropic vortices aligned along the step edges. BKT physics using in situ transport could be studied in monolayer superconductors going beyond previous experiments although some issues remain to be clarified ${ }^{108}$.

In the light of recent measurements, it thus appears that these superconducting $2 \mathrm{D}$ monolayers made of heavy elements are prototypical systems to study p-wave superconductivity. Much more experimental and theoretical work is needed to characterize in details this p-wave component and properties in the diffusive limit encountered in the experiment. In particular we have to study how the s- and p- components live together, what are their respective amplitudes and how the tunneling process probes the excitations of both components. The anisotropy of the bandstructure might play an important role, considering for example the noticeable differences existing between the SIC and $\sqrt{7} \times \sqrt{3}$-Pb MLs regarding the presence of in-gap states induced by nonmagnetic disorder. Moreover we want to know which parts of the bandstructure contribute to which superconducting component and so on, taking into account that, up to now, single energy gaps have been measured in such systems.

2D monolayer superconductors made of heavy elements offer a very exciting and timely platform to study topological superconductivity thanks to their p-wave component due to strong $\mathrm{SOC}^{169}$. As recently proposed and realized, coupling such systems to controlled and organized local magnetic structures grown in situ will enable fabricating and tuning various superconducting topological phases ${ }^{170}$, while allowing at the same time for a full $a b$ initio-based description of their electronic properties. This should step forward the study of Majorana fermions physics using better controlled systems, the interface between a trivial and a topological superconducting phase being for instance one-dimensional ${ }^{170}$ instead of zero-dimensional ${ }^{171,172}$.

\section{ACRONYMS}

DOS: density of states

STM: scanning tunneling microscopy

STS: scanning tunneling spectroscopy

ARPES: angular resolved photoemission spectroscopy MBE: molecular beam epitaxy

UHV: ultrahigh vacuum

2DEG: two-dimensional electron gas

LEED: low-energy electron diffraction

RHEED: reflection high-energy electron diffraction

XPS: x-ray photoemission spectroscopy

SXRD: surface x-ray diffraction

DFT: density functional theory

u.c.: unit cell

SOC: spin-orbit coupling

$T_{c}$ : critical temperature

$\Delta$ : energy gap

$k_{F}$ : Fermi wavevector

$\lambda_{F}$ : Fermi wavelength

$E_{F}$ : Fermi energy

\section{ACKNOWLEDGMENTS}

We thank G. Ménard, M. Tringides, L. Ioffe, H. Pothier, J.C. Cuevas, M. Milosevic, N. Bergeal, J. Biscaras for useful discussions. We are grateful to G. Ménard for his critical reading of the manuscript. Also, we would like to mention that G. Ménard performed together with the authors the unpublished experiments presented in Fig.17 panels e,f,g. The numerous recent works from the authors of this review paper were supported by grants from the ANR French National funding Agency. C.B., T.C. and D.R acknowledge financial support from the ANR MISTRAL, SUPERSTRIPES and RODESIS. Financial support from the Émergence program of the University Pierre et Marie Curie is also acknowledged.
* Electronic address: christophe.brun@upmc.fr

1 Ginzburg, V. L. \& Kirzhnits, D. A. On the superconductivity of electrons at the surface levels. Zh. Exp. Teor. Fiz. 46, 397 (1964). Sov. Phys. - JETP, 19, 269 (1964).

2 Ginzburg, V. L. On surface superconductivity. Phys. Lett. 13, 101 (1964).
${ }^{3}$ Little, W. A. Possibility of Synthesizing an Organic Superconductor. Phys. Rev. 134, A1416 (1964).

${ }^{4}$ Buckel, V. and Hilsch, R. Einflu der Kondensation bei tiefen Temperaturen auf den elektrischen Widerstand und die Supraleitung fr verschiedene Metalle. Z. Phys. 138109 (1954). 
${ }^{5}$ Ohtomo, A. and Hwang, H. Y. A high-mobility electron gas at the $\mathrm{LaAlO}_{3} / \mathrm{SrTiO}_{3}$ heterointerface. Nature 427, 423 (2004).

6 Reyren, N. et al.. Superconducting interfaces between insulating oxides. Science 317, 1196 (2007).

7 Gariglio S. et al. chapter 7 surface and interface superconductivity, in The Oxford handbook of small superconductors. Oxford University Press. to appear (2016).

8 Zhang, T. et al. Superconductivity in one-atomic-layer metal films grown on $\mathrm{Si}(111)$. Nature Phys. 6, 104 (2010).

9 Ye, J.T. et al. Superconducting Dome in a Gate-Tuned Band Insulator. Science 338, 1193 (2012).

10 Cui, X. et al. Multi-terminal transport measurements of $\mathrm{MoS}_{2}$ using a van der Waals heterostructure device platform. Nature Mat. 10, 534 (2015).

11 Saito, Y. et al. Superconductivity protected by spin-valley locking in ion-gated $\mathrm{MoS}_{2}$. Nature Phys. 12, 144 (2015).

${ }^{12} \mathrm{Xi}, \mathrm{X}$. et al. Ising pairing in superconducting $\mathrm{NbSe} 2$ atomic layers. Nature Phys. 12, 139 (2016).

13 Tsen, A.W. et al. Nature of the quantum metal in a twodimensional crystalline superconductor. Nature Phys. 12, 208 (2016).

${ }^{14}$ Costanzo, D. et al. Gate-induced superconductivity in atomically thin $\mathrm{MoS}_{2}$ crystals. Nature Nanotech. 11, 339 (2016).

15 Ueno, K. et al. Electric-field-induced superconductivity in an insulator. Nature Mater. 7, 855 (2008).

16 Ye, J.T. et al. Liquid-gated interface superconductivity on an atomically flat film. Nature Mater. 9, 125 (2010).

17 Allain, A., Han, Z. and Bouchiat, V. Electrical control of the superconducting-to-insulating transition in graphenemetal hybrids. Nature Mater. 11, 590 (2012).

18 Han, Z. et al. Collapse of superconductivity in a hybrid tingraphene Josephson junction array. Nature Phys. 10, 380 (2014).

19 Dubuis, G., Bollinger, A.T. Pavuna, D. and Bosovic, I. On field effect studies and superconductor-insulator transition in high-Tc cuprates. Eur. Phys. J. Special Topics 222, 1217 (2013).

20 Wang, Q.-Y. et al. Interface-Induced High-Temperature Superconductivity in Single Unit-Cell FeSe Films on $\mathrm{SrTiO}_{3}$. Chinese Phys. Lett. 29, 037402 (2012).

${ }^{21} \mathrm{Ge}$, J.-F. et al. Superconductivity above $100 \mathrm{~K}$ in singlelayer FeSe films on doped $\mathrm{SrTiO}_{3}$. Nature Mat. 14, 285 (2014).

22 Abrikosov, A.A. \& Gorkov, L.P. On the theory of superconducting alloys: 1 . The electrodynamics of alloys at absolute zero. JETP 8, 1090 (1958)

23 Anderson, P. W. Theory of dirty superconductors. J. Phys. Chem. Solids 11, 26 (1959).

${ }^{24}$ Goldman, A.M. \& Markovic, N. Superconductor-insulator transitions in the two-dimensional limit. Physics Today. 51, 11, 39 (1998)

25 Feigel'man, M.V. Ioffe, L.B. Kravtsov, V.E. \& Cuevas, E. Fractal superconductivity near localization threshold. Annals of Physics 325 1390-1478 (2010).

${ }^{26}$ Gantmakher, V.F. \& Dolgopolov, V.T. Superconductorinsulator quantum phase transition. Physics - Uspekhi 53 1-49 (2010).

27 Grabert, H. \& Devoret, M.H. Single Charge Tunneling: Coulomb Blockade Phenomena in Nanostructures. Plenum, New York and London (1992).

28 Efetov, K.B. Phase transition in granulated superconductors. Sov. Phys. JETP 51, 1015 (1980).
${ }^{29}$ Orr, B.G., Jaeger, H.M. and Goldman, A.M. Local superconductivity in ultrathin Sn films. Phys. Rev. B 32, 7586 (1985).

30 White, A.E., Dynes, R.C. and Garno, J.P. Destruction of superconductivity in quench-condensed two-dimensional films. Phys. Rev. B 33, 3549 (1986).

31 Jaeger, H.M. et al.. Threshold for superconductivity in ultrathin amorphous gallium films. Phys. Rev. B 34, 4920 (1986).

32 Graybeal, J.M. and Beasley, M.R. Localization and interaction effects in ultrathin amorphous superconducting films. Phys. Rev. B 29, 4167 (1984).

33 Dynes, R.C., White, A.E., Graybeal, J.M. and Garno, J.P. Breakdown of Eliashberg Theory for TwoDimensional Superconductivity in the Presence of Disorder. Phys. Rev. Lett. 57, 2195 (1986).

${ }^{34}$ Haviland, D.B. et al. Onset of superconductivity in the two-dimensional limit. Phys. Rev. Lett. 62, 2180 (1989).

35 Finkel'stein, A.M. Superconducting transition temperature in amorphous films. Pisma ZhETF 45, 37 (1987) [JETP Letters 45, 46 (1987)].

36 Altshuler, B.L., Aronov, A.G., Lee, P.A. Interaction Effects in Disordered Fermi Systems in Two Dimensions. Phys. Rev. Lett. 44, 1288 (1980).

37 Braun, F., Von Delft, J. Spectroscopy of discrete energy levels in ultrasmall metallic grains. Phys. Rep. 345, 61 (2001).

38 Bose, S. and Ayyub, P.A review of finite size effects in quasi-zero dimensional superconductors. Rep. Prog. Phys. 77, 116503 (2014).

39 Sacépé, B. et al. Disorder-Induced Inhomogeneities of the Superconducting State Close to the SuperconductorInsulator Transition. Phys. Rev. Lett. 101, 157006 (2008).

40 Noat, Y. et al. Unconventional superconductivity in ultrathin superconducting NbN films studied by scanning tunneling spectroscopy. Phys. Rev. B 88, 014503 (2013).

41 Chand, M. et al. Phase diagram of the strongly disordered s-wave superconductor $\mathrm{NbN}$ close to the metal-insulator transition Phys. Rev. B 85, 014508 (2012).

${ }^{42}$ Kamlapure, A. et al. Emergence of nanoscale inhomogeneity in the superconducting state of a homogeneously disordered conventional superconductor. Sci. Rep. 3, 2979; DOI:10.1038/srep02979 (2013).

${ }^{43}$ Carbillet, C. et al. Confinement of superconducting fluctuations due to emergent electronic inhomogeneities. Phys. Rev. B 93, 144509 (2016).

44 Dynes, R.C., Narayanamurti, V. and Garno J.P. Direct Measurement of Quasiparticle-Lifetime Broadening in a Strong-Coupled Superconductor. Phys. Rev. Lett. 41, 1509 (1978).

45 Burmistrov, I.S., Gornyi, I.V. and Mirlin A.D. Local density of states and its mesoscopic fluctuations near the transition to a superconducting state in disordered systems. Phys. Rev. B 93, 205432 (2016).

46 Sacépé, B. et al.. Localization of preformed Cooper pairs in disordered superconductors. Nat. Phys. 7, 239 (2011).

47 Sacépé, B. et al.. Pseudogap in a thin film of a conventional superconductor. Nat. Commun. 101, 157006 (2010).

48 Gol'tsman, G.N. et al. Picosecond superconducting singlephoton optical detector. Appl. Phys. Lett. 79, 705 (2001).

49 Annunziata, A.J. et al. Tunable superconducting nanoinductors. Nanotechnology 21, 445202 (2010).

${ }^{50}$ Guo, Y. et al. Superconductivity modulated by quantum 
size effects. Science 306, 1915 (2004).

51 Bao, X.-Y. et al. Quantum Size Effects on the Perpendicular Upper Critical Field in Ultrathin Lead Films. Phys. Rev. Lett. 95, 247005 (2005).

52 Özer, M.M., Thompson, J.R. \& Weitering, H.H. Hard superconductivity of a soft metal in the quantum regime. Nature Phys. 2, 173 (2006).

53 Eom, D. Qin, S. Chou, M.-Y. \& Shih, C. K. Persistent superconductivity in ultrathin Pb films: a scanning tunneling spectroscopy study. Phys. Rev. Lett. 96, 027005 (2006).

54 Özer, M.M., Jia, Y., Zhang, Z., Thompson J. R. \& Weitering, H. H. Tuning the quantum stability and superconductivity of ultrathin metal alloys. Science 316, 1594 (2007).

55 Brun, C. et al. Reduction of the superconducting gap of ultrathin $\mathrm{Pb}$ islands grown on $\mathrm{Si}(111)$. Phys. Rev. Lett. 102, 207002 (2009).

${ }^{56}$ Qin, S.Y., Kim, J., Niu, Q. \& Shih, C.K. Superconductivity at the two-dimensional limit. Science 324, 1314-1317 (2009).

57 Strongin, M., Kammerer, O. F., Farrell, H.H. and Miller, D. L. Superconducting Properties of Crystalline Ultrathin Films. Phys. Rev. Lett. 30, 129 (1973).

58 Orr, B.G., Jaeger, H.M., and Goldman, A.M. Transitiontemperature oscillations in thin superconducting films. Phys. Rev. Lett. 53, 2046 (1984).

59 Blatt, J.M., and Thomson, C.J. Shape resonnances in superconducting thin films. Phys. Rev. Lett. 10, 332 (1963).

60 Blatt, J.M., and Thomson, C.J. Shape resonnances in superconductors - II Simplified theory. Physics Lett. 5, 6 (1963).

61 Altfeder, I.B. et al. Electron Fringes on a Quantum Wedge. Phys. Rev. Lett. 78, 2815 (1997).

62 Budde, K. et al. Uniform, self-organized, seven-step height $\mathrm{Pb} / \mathrm{Si}(111)(77)$ islands at low temperatures. Phys. Rev. B 61, $10602 \mathrm{R}$ (2000).

${ }^{63}$ Feng, R. et al. Wetting-layer transformation for $\mathrm{Pb}$ nanocrystals grown on $\mathrm{Si}(111)$. Appl. Phys. Lett. 85, 3866 (2004).

64 T.-C. Chiang. Photoemission studies of quantum well states in thin films. Surf. Sci. Rep. 39, 181 (2000).

65 J.H. Dil, J. W. Kim, T. Kampen, K. Horn, and A.R.H.F. Ettema. Electron localization in metallic quantum wells: $\mathrm{Pb}$ versus $\mathrm{In}$ on $\mathrm{Si}(111)$. Phys. Rev. B 73, $161308 \mathrm{R}$ (2006).

66 P.S. Kirchmann, M. Wolf, J.H. Dil, K. Horn, and U. Bovensiepen. Quantum-size effects in $\mathrm{PbSi}(111)$ investigated by laser-induced photoemission. Phys. Rev. B 76, 075406 (2007).

67 I.P. Hong et al. Decay mechanisms of excited electrons in quantum-well states of ultrathin $\mathrm{Pb}$ islands grown on $\mathrm{Si}(111)$ : Scanning tunneling spectroscopy and theory. Phys. Rev. B 80, 081409 R (2009).

68 C.M. Wei and M.Y. Chou. Theory of quantum size effects in thin $\mathrm{Pb}(111)$ films. Phys. Rev. B 66, 233408 (2002).

69 Hinch, B. J.,Koziol, C., Toennies, J.P. and Zhang, G. Evidence for Quantum Size Effects Observed by Helium Atom Scattering during the Growth of $\mathrm{Pb}$ on $\mathrm{Cu}(111)$. Europhys. Lett. 10, 341 (1989).

70 Zhang, Z., Niu Q. and Shih, C.-K. Electronic growth of metallic overlayers on semiconductor substrates. Phys. Rev. Lett. 80, 5381 (1998).
${ }^{71} \mathrm{Su}, \mathrm{W} . \mathrm{B}$. et al. Correlation between quantized electronic states and oscillatory thickness relaxations of $2 \mathrm{D} \mathrm{Pb}$ islands on Si(111)-(7x7) surfaces. Phys. Rev. Lett. 86, 5116 (2001).

72 Hupalo, M. and Tringides, M.C. Correlation between height selection and electronic structure of the uniform height $\mathrm{Pb} / \mathrm{Si}(111)$ islands. Phys. Rev. Lett. 65, 115406 (2002).

$73 \mathrm{Su}$, W.B., Chang, C.S. and Tsong, T.T. Quantum size effect on ultra-thin metallic films. J. Phys. D: Appl. Phys. 43, 013001 (2010).

74 Ma, L.-Y. et al. Quantum Size Effect on Adatom Surface Diffusion. Phys. Rev. Lett. 97, 266102 (2006).

75 Shanenko, A.A., Croitoru, M.D. and Peeters, F.M. Oscillations of the superconducting temperature induced by quantum well states in thin metallic films : Numerical solution of the Bogoliubov-de Gennes equations. Phys. Rev. $B$ 75, 014519 (2007).

${ }^{76} \mathrm{Fu}$, Y.-S. Manipulating the Kondo Resonance through Quantum Size Effects. Phys. Rev. Lett. 99, 256601 (2007).

77 O. Pfennigstorf, A. Petkova, H.L. Guenter, and M. Henzler. Conduction mechanism in ultrathin metallic films. Phys. Rev. B 65, 045412 (2002).

78 Noffsinger, J. and Cohen, M.L. First-principles calculation of the electron-phonon coupling in ultrathin $\mathrm{Pb} \mathrm{su}-$ perconductors: Suppression of the transition temperature by surface phonons. Phys. Rev. B 81, 214519 (2010).

79 Schackert, M. et al. Local Measurement of the Eliashberg Function of Pb Islands: Enhancement of Electron-Phonon Coupling by Quantum Well States. Phys. Rev. Lett. 114, 047002 (2015).

80 Valentinis, D., Van der Marel, D., and Berthod, C. BCS superconductivity near the band edge: Exact results for one and several bands. Phys. Rev. B 94, 024511 (2016).

81 Valentinis, D., Van der Marel, D., and Berthod, C. Rise and fall of shape resonances in thin films of BCS superconductors. Phys. Rev. B 94, 054516 (2016).

${ }^{82}$ Nishio, T. et al. Superconducting Pb Island Nanostructures Studied by Scanning Tunneling Microscopy and Spectroscopy. Phys. Rev. Lett. 101, 167001 (2008).

${ }^{83}$ Cren, T. et al. Ultimate vortex confinement studied by scanning tunneling spectroscopy. Phys. Rev. Lett. 102, 127005 (2009).

${ }^{84}$ Ning, Y.X., et al. Vortex properties of two-dimensional superconducting films. J. Phys.: Condens. Matter 22065701 (2010).

85 Cren, T. et al. Vortex fusion and giant vortex states in confined superconducting condensatesPhys. Rev. Lett. 107, 097202 (2011).

86 Seehofer, L. Falkenberg, G. Daboul, D. \& Johnson, R.L. Structural study of the close-packed two-dimensional phases of $\mathrm{Pb}$ on $\mathrm{Ge}(111)$ and $\mathrm{Si}(111)$. Phys. Rev. B, 51, 13503 (1995).

87 Hupalo, M. et al. Atomic models, domain-wall arrangement, and electronic structure of the dense $\mathrm{Pb} / \mathrm{Si}(111)$ $\sqrt{3} \times \sqrt{3}$ phase. Phys. Rev. B 66, 161410R (2002).

${ }^{88}$ Hupalo, M., Schmalian, J. \& Tringides, M.C. Devil staircase in $\mathrm{Pb} / \mathrm{Si}(111)$ ordered phases. Phys. Rev. Lett. 90, 216106 (2003).

${ }^{89}$ Horikoshi, K. Tong, X. Nagao, T. \& Hasegawa S. Structural phase transitions of $\mathrm{Pb}$-adsorbed $\mathrm{Si}(111)$ surfaces at low temperatures. Phys. Rev. B 60, 13287 (1999).

90 de Vries, S.A. et al. Phase transition of a $\mathrm{Pb}$ monolayer on Ge(111). Phys. Rev. B 59, 13301 (1999). 
${ }^{91}$ Kumpf, C. et al. Structural study of the commensurate incommensurate low-temperature phase transition of $\mathrm{Pb}$ on $\mathrm{Si}(111)$. Surf. Sci. 448, L213-L219 (2000).

92 Brochard, S. et al. ab initio calculations and scanning tunneling microscopy experiments of the $\operatorname{Si}(111) \sqrt{7} \times \sqrt{3}$ Pb surface. Phys. Rev. B, 66, 205403 (2002).

93 Ren, X.-Y. et al. Spin-orbit coupling effects on the stability of two competing structures in $\mathrm{Pb} / \mathrm{Si}(111)$ and $\mathrm{Pb} / \mathrm{Ge}(111)$. arXiv:1606.04208v1 (2016).

${ }^{94}$ Chan, T.-L. et al. First-principles studies of structures and stabilities of $\mathrm{Pb} / \mathrm{Si}(111)$. Phys. Rev. B, 68, 045410 (2003).

95 Stepanovsky, S. et al. The dense $\alpha-\sqrt{3} \times \sqrt{3} \mathrm{~Pb} / \mathrm{Si}(111)$ phase: A comprehensive STM and SPA-LEED study of ordering, phase transitions and interactions. Surf. Sci., 600, 1417 (2006)

${ }^{96}$ Choi, W.H., Koh, H., Rotenberg, E. \& Yeom, H.W. Electronic structure of dense $\mathrm{Pb}$ overlayers on $\mathrm{Si}(111)$ investigated using angle-resolved photoemission. Phys. Rev. B 75, 075329 (2007).

97 Kim, K.S., Jung, S.C., Kang, M.H. \& Yeom, H.W. Nearly massless electrons in the silicon interface with a metal film. Phys. Rev. Lett. 104, 246803 (2010).

98 Jung, S.C. \& Kang, M.H. Triple-domain effects on the electronic structure of $\mathrm{Pb} / \mathrm{Si}(111)-(\sqrt{7} \times \sqrt{3})$ : Densityfunctional calculations. Surf. Sci. 605, 551 (2011).

99 Uchihashi, T., Puneet, M., Aono, M.,\& Nakayama, T. Macroscopic superconducting current through a silicon surface reconstruction with indium adatoms: $\mathrm{Si}(111)$ $(\sqrt{7} \times \sqrt{3})$-In. Phys. Rev. Lett. 107, 207001 (2011).

100 Yamada, Y., Hirahara, T., \& Hasegawa, S. Magnetoresistance measurements of a superconducting surface state of In-induced and $\mathrm{Pb}$-induced structures on $\mathrm{Si}(111)$. Phys. Rev. Lett. 110, 237001 (2013).

101 Tanikawa, T. et al. Variable-Temperature Micro-FourPoint Probe Method for Surface Electrical Conductivity Measurements in Ultrahigh Vacuum. e-J. Surf. Sci. Nanotechnol. 1, 50 (2003).

102 Kraft, J. et al. Surface reconstructions of In on $\mathrm{Si}(111)$. Phys. Rev. B 55, 5384 (1997).

103 Park, J.W. and Kang, M.H. Double-Layer In Structural Model for the $\mathrm{In} / \mathrm{Si}(111)-\sqrt{7} \times \sqrt{3}$ Surface.Phys. Rev. Lett. 109, 166102 (2012).

104 Park, J.W. and Kang, M.H. Hexagonal indium doublelayer on $\mathrm{Si}(111)-\sqrt{7} \times \sqrt{3}$. Phys. Rev. B 92, 045306 (2015).

105 Park, J.W. and Kang, M.H. Single-layer limit of metallic indium overlayers on Si(111).Phys. Rev. Lett. 117, 116102 (2016).

106 Larkin, A. and Varlamov, A. Theory of fluctuations in superconductors. Oxford University, New York, 2005.

107 Hsu, J.W. and Kapitulnik, A. Superconducting transition, fluctuation, and vortex motion in a two-dimensional single-crystal Nb film. Phys. Rev. B 45, 4819 (1992).

108 Matetskiy, A.V., et al. Two-Dimensional Superconductor with a Giant Rashba Effect: One-Atom-Layer Tl$\mathrm{Pb}$ Compound on $\mathrm{Si}(111)$. Phys. Rev. Lett. 115, 147003 (2015).

109 Gruznev, D.V., et al. A Strategy to Create Spin-Split Metallic Bands on Silicon Using a Dense Alloy Layer. Sci. Rep. 4, 4742 (2014).

110 Ambegaokar, V. and Baratoff, A. Tunneling Between Superconductors. Phys. Rev. Lett. 10, 486 (1963).

111 Matsuda, I. et al. Electrical Resistance of a Monatomic Step on a Crystal Surface. Phys. Rev. Lett. 93, 236801
(2004).

112 Brun, C. et al. Remarkable effects of disorder on superconductivity of single atomic layers of lead on silicon. Nature Phys. 10, 444 (2014).

113 Yoshizawa, S. et al. Imaging Josephson Vortices on the Surface Superconductor $\operatorname{Si}(111)-\sqrt{7} \times \sqrt{3}$-In using a Scanning Tunneling Microscope. Phys. Rev. Lett. 113, 247004 (2014).

114 Sekihara, T. et al. Two-Dimensional Superconducting State of Monolayer $\mathrm{Pb}$ Films Grown on $\mathrm{GaAs}(110)$ in a Strong Parallel Magnetic Field. Phys. Rev. Lett. 111, 057005 (2013).

115 Clogston, A.M. Upper limit for the critical field in hard superconductor. Phys. Rev. Lett. 9, 266 (1962).

116 Chandrasekhar, B.S. A note on the maximum critical field of high-field superconductors. Appl. Phys. Lett. 1, 7 (1962).

117 Edelstein, V.M. Characteristics of the Cooper pairing in two-dimensional noncentrosymmetric electron systems. Zh. Eksp. Teor. Fiz. 95, 2151 (1989) [Sov. Phys. JETP 68, 1244 (1989)].

118 Gor'kov, L. P., and Rashba, E. I. Superconducting 2D System with Lifted Spin Degeneracy: Mixed SingletTriplet State. Phys. Rev. Lett. 87, 037004 (2001).

119 Bychkov, Yu.A. and Rashba, E.I. Properties of a 2D electron gas with lifted spectral degeneracy. Sov. Phys. JETP Lett. 39, 78 (1984).

${ }^{120}$ LaShell, S. et al. Spin Splitting of an Au(111) Surface State Band Observed with Angle Resolved Photoelectron Spectroscopy. Phys. Rev. Lett. 77, 3419 (1996).

121 Gierz, I., et al. Silicon Surface with Giant Spin Splitting. Phys. Rev. Lett. 103, 046803 (2009).

122 Hatta, S., et al. Large Rashba spin splitting of surface resonance bands on semiconductor surface. Phys. Rev. B 80, 113309 (2009).

123 Sakamoto, K., et al. Peculiar Rashba Splitting Originating from the Two-Dimensional Symmetry of the Surface. Phys. Rev. Lett. 103, 156801 (2009).

124 Yaji, K., et al. T. Large Rashba spin splitting of a metallic surface-state band on a semiconductor surface. Nat. Commun. 1:17 doi: 10.1038/ncomms1016 (2010).

125 Barzykin, V. and Gor'kov, L. P. Inhomogeneous Stripe Phase Revisited for Surface Superconductivity. Phys. Rev. Lett. 89, 227002 (2002).

126 Fulde, P. and Ferell, R.A. Superconductivity in a Strong Spin-Exchange Field. Phys. Rev. 135, A550 (1964).

127 Larkin, A.I. and Ovchinnikov, Yu.N. Nonuniform state of superconductors. Zh. Eksp. Teor. Fiz. 47, 1136 (1964) [Sov. Phys. JETP 20, 762 (1965)].

128 Dimitrova, O.V. and Feigel'man, M.V. Phase Diagram of a Surface Superconductor in Parallel Magnetic Field. JETP Lett. 78, 637 (2003).

129 Dimitrova, O.V. and Feigel'man, M.V. Theory of a twodimensional superconductor with broken inversion symmetry. Phys. Rev. B 76, 014522 (2007).

130 Mermin, N.D. and Wagner, H. Absence of Ferromagnetism or Antiferromagnetism in One- or TwoDimensional Isotropic Heisenberg Models. Phys. Rev. Lett. 17, 1133 (1966).

131 Berezinskii, V.L. Destruction of Long-range Order in One dimensional and Two-dimensional Systems Possessing a Continuous Symmetry Group. II. Quantum Systems. [Zh. Eksp. Teor. Fiz., 61, 1144] J. Exp. Theor. Phys. 34, 610 (1972). 
132 Kosterlitz, J.M., and Thouless, D.J. Long range order and metastability in two dimensional solids and superfluids. (Application of dislocation theory). J. Phys. C Solid State Phys. 5, L124 (1972).

133 Halperin, B.I., and Nelson, D.R. Resistive transition in superconducting films. J. Low Temp. Phys. 36, 599 (1979).

134 Doniach, S. and Huberman, B. A. Topological Excitations in Two-Dimensional Superconductors. Phys. Rev. Lett. 42, 1169 (1979).

135 Bishop, D.J. and Reppy, J.D. Study of the superfluid transition in two-dimensional ${ }^{4}$ He films. Phys. Rev. Lett. 40, 1727 (1978).

136 Agnolet, G., McQueeney, D.F. and Reppy, J.D. Kosterlitz-Thouless transition in helium films. Phys. Rev. B 39, 8934 (1989).

137 Minnhagen, P. The two-dimensional Coulomb gas, vortex unbinding, and superfluid-superconducting films. Rev. Mod. Phys. 59, 1001 (1987).

138 Benfatto, L., Castellani, C., and Giamarchi, T. (2013). Beresinskii-Kosterlitz-Thouless transition within the sineGordon approach: the role of the vortex-core energy. In 40 Years of Berezinskii-Kosterlitz-Thouless Theory (ed. Jos, J. V.). Scientific World.

139 Beasley, M.R., Mooij, J.E. and Orlando, T.P. Possibility of vortex-antivortex pair dissociation in two-dimensional superconductorsPhys. Rev. Lett. 42, 1165 (1979).

140 Gabay, M, and Kapitulnik, A. Vortex-antivortex crystallization in thin superconducting and superfluid films. Phys. Rev. Lett. 71, 2138 (1993).

141 Zhao, W, et al.. Evidence for Kosterlitz-Thouless transition in atomically flat two-dimensional $\mathrm{Pb}$ superconducting films. Solid State Commun. 165, 59 (2013).

142 Zhang, H.-M., et al.. Detection of a superconducting phase in a two-atom layer of hexagonal Ga films grown on semiconducting GaN(0001).Phys. Rev. Lett. 114, 107003 (2015).

143 Nelson, D.R. and Kosterlitz, J.M. Universal Jump in the Superfluid Density of Two-Dimensional Superfluids. Phys. Rev. Lett. 39, 1201 (1977).

144 Bouadim, K., Loh, Y.L., Randeria, M. \& Trivedi, N. Single- and two-particle energy gaps across the disorderdriven superconductor-insulator transition. Nature Phys. 7, 884 (2011)

145 Hetel, I., Lemberger, T.R. and Randeria, M. Quantum critical behaviour in the superfluid density of strongly underdoped ultrathin copper oxide films. Nature Phys. 3, 700 (2007).

146 Cherkez, V. et al. Proximity Effect between Two Superconductors Spatially Resolved by Scanning Tunneling Spectroscopy. Phys. Rev. X 4, 011033 (2014).

$147 \mathrm{Yu}, \mathrm{L}$. Bound state in superconductors with paramagnetic impurities. Acta Phys. Sin. 21, 75 (1965).

148 Shiba, H. Classical spins in superconductors. Prog. Theor. Phys. 40, 435 (1968).

149 Rusinov, A.I. Superconductivity near a paramagnetic impurity. JETP Lett. 9, 85 (1969).

150 Bauriedl,W., Ziemann, P. \& Buckel,W. Electrontunneling observation of impurity bands in superconducting manganese-implanted lead. Phys. Rev. Lett. 47, 1163 (1981).

151 Balatsky, A.V., Vekhter, I. \& Zhu, J.-X. Impurity-induced states in conventional and unconventional superconduc- tors. Rev. Mod. Phys. 78, 373 (2006).

152 Yazdani, A., Jones, B.A., Lutz, C.P., Crommie, M.F. \& Eigler, D.M. Probing the local effects of magnetic impurities on superconductivity. Science 275, 1767 (1997).

$153 \mathrm{Ji}$, S.-H. et al. High-resolution scanning tunneling spectroscopy of magnetic impurity induced bound states in the superconducting gap of $\mathrm{Pb}$ thin films. Phys. Rev. Lett. 100, 226801 (2008).

154 Franke, K.J., Schulze, G. \& Pascual, J.I. Competition of superconducting phenomena and Kondo screening at the nanoscale. Science 332, 940 (2011).

155 Ménard, G.C. et al. Coherent long-range magnetic bound states in a superconductor. Nature Phys. 11, 1013 (2015).

156 Mackenzie, A.P. et al. Extremely Strong Dependence of Superconductivity on Disorder in $\mathrm{Sr}_{2} \mathrm{RuO}_{4}$. Phys. Rev. Lett. 80, 161 (1998).

157 Maki, K. and Puchkaryov, E. Impurity effects in p-wave superconductors. Europhys. Lett. 50, 533 (2000).

$158 \mathrm{Kim}, \mathrm{H}$. et al. Superconducting proximity effect on a Rashba-split $\mathrm{Pb} / \mathrm{Ge}(111)-\beta \sqrt{3} \times \sqrt{3}$ surface. Supercond. Sci. Technol. 29, 084006 (2016).

159 Yang, Z.H., Wang, J. and Chan, K.S. Proximity effect in a superconductor/two-dimensional electron gas junction with Rashba spin-orbit coupling. Supercond. Sci. Technol. 22, 055012 (2009).

160 Reeg, C.R., and Maslov, D.L. et al. Proximity-induced triplet superconductivity in Rashba materials. Phys. Rev. B 92, 134512 (2015).

161 Kim, H. et al. Electrical Conductivity through a single atomic step measured with the proximity-induced superconducting pair correlation. Phys. Rev. Lett. 117, 116802 (2016).

$162 \mathrm{Kim}, \mathrm{H}$. et al. Visualization of geometric influences on proximity effects in heterogeneous superconductor thin films. Nat. Phys. 8, 464 (2012).

163 Serrier-Garcia, L. et al. Scanning Tunneling Spectroscopy Study of the Proximity Effect in a Disordered TwoDimensional Metal. Phys. Rev. Lett. 110, 157003 (2013).

164 De Gennes, P.G. (ed.) Superconductivity of Metals and Alloys (W A Benjamin, 1966).

165 Pearl, J. Current distribution in superconducting films carrying quantized fluxoids. Appl. Phys. Lett. 5, 65 (1964).

166 Gurevich, A. Nonlocal Josephson electrodynamics and pinning in superconductors. Phys. Rev. B 463187 (1992).

167 Roditchev, D. et al. Direct observation of Josephson vortex cores. Nature Phys. 11, 332 (2015).

168 Nam, H. et al. Ultrathin two-dimensional superconductivity with strong spin-orbit coupling. Pnas early edition (2016).

169 Beenakker, C. and Kouvenhowen L.A road to reality with topological superconductors. Nature Phys. 12, 618 (2016).

170 Ménard, G.C. et al. Topological superconductivity in $\mathrm{Pb} / \mathrm{Co} / \mathrm{Si}(111)$ monolayer. arXiv:1607.06353v1 (2016).

${ }^{171}$ Mourik, V. et al. Signatures of Majorana fermions in hybrid superconductorsemiconductor nanowire devices. Science 336, 1003 (2012).

172 Nadj-Perge, S. et al. Observation of Majorana fermions in ferromagnetic atomic chains on a superconductor. Science 346, 602 (2014). 

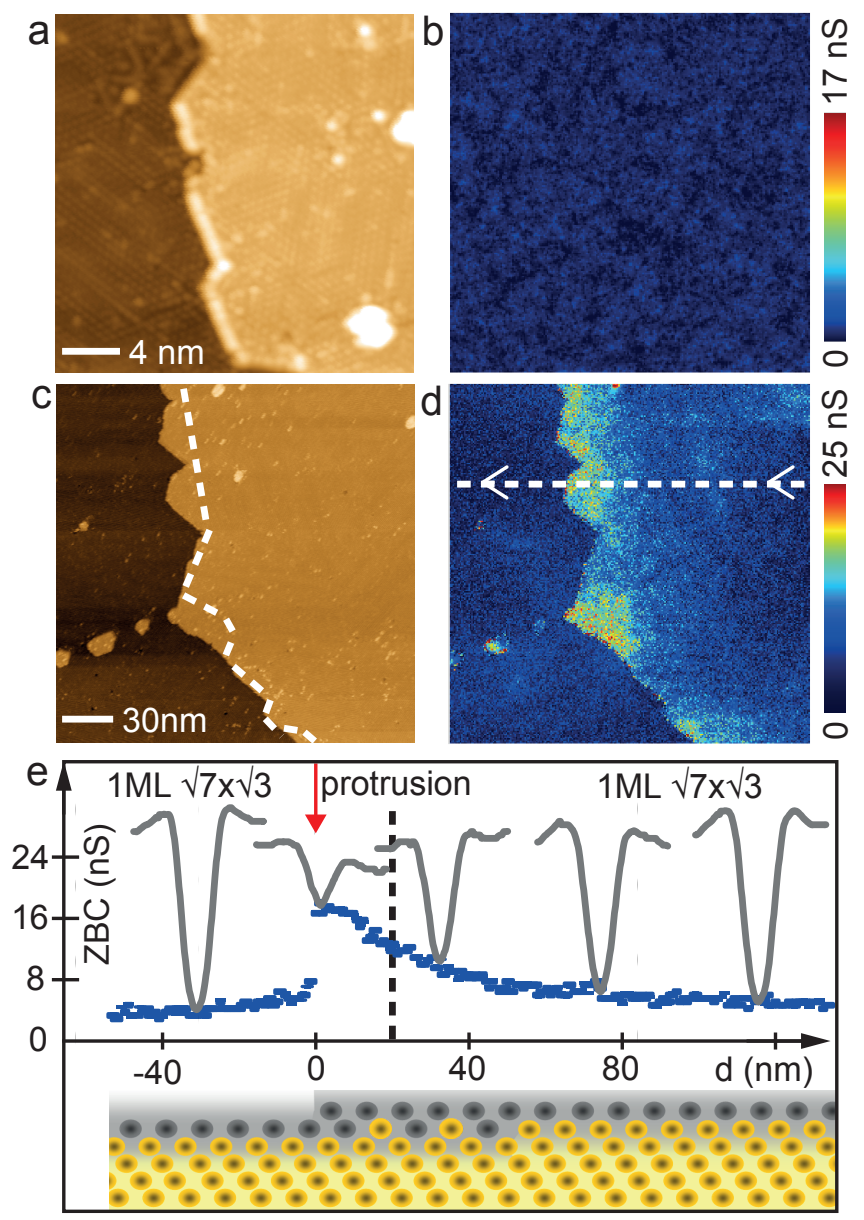

FIG. 20: Different superconducting behaviors of atomic step edges in $\mathrm{Pb}$ monolayers. Effect of a monoatomic step edge on the superconductivity of the SIC$\mathrm{Pb}(\mathrm{a}, \mathrm{b})$ and $\sqrt{7} \times \sqrt{3}$ - $\mathrm{Pb}(\mathrm{c}, \mathrm{d}, \mathrm{e})$ monolayers. Combined with magnetic field measurements (see Fig. 21) it shows that a monoatomic step is a Josephson junction in $\sqrt{7} \times \sqrt{3}$ while it is highly transparent in SIC. a),c): Respective topographic images of the SIC $\left(26 \times 26 \mathrm{~nm}^{2}\right)$ and $\sqrt{7} \times \sqrt{3}\left(210 \times 210 \mathrm{~nm}^{2}\right)$ phases centred at single atomic step edges. The dashed line in c) delimits the separation between nano-protrusions (see text) and the upper terrace to which they are attached. b),d): Corresponding zero-bias conductance maps. For the SIC sample (b), the local tunnelling spectra exhibit a well-developed superconducting gap across the step edge, which is indistinguishable in the spectroscopic map. For the $\sqrt{7} \times \sqrt{3}$ sample (d), the nano-protrusions exhibit a high zero-bias conductance and thus are not superconducting. In the vicinity of the nano-protrusions, the upper terrace shows a spatially dependent zero-bias conductance due to the inverse proximity effect, the superconducting gap being restored on a scale of several tens of nanometres. On the lower terrace, the gap abruptly reopens in the immediate vicinity of the step edge close to the nano-protrusion. e): Spatial evolution of the zerobias conductance (ZBC) along the dashed line shown in d), when going from the upper to the lower terrace through a nano-protrusion. The lateral extent of the nano-protrusion is between the red arrow $(0 \mathrm{~nm})$ and the black dashed line (20 $\mathrm{nm}$ ) as seen in inferred from panel c). Selected $\mathrm{dI}=\mathrm{dV}$ spectra measured along this dashed line are shown in real conductance units. Yellow and grey discs schematically depict silicon and lead atoms in the vicinity of the step edge. Reproduced after Brun et al. ${ }^{112}$ 
a

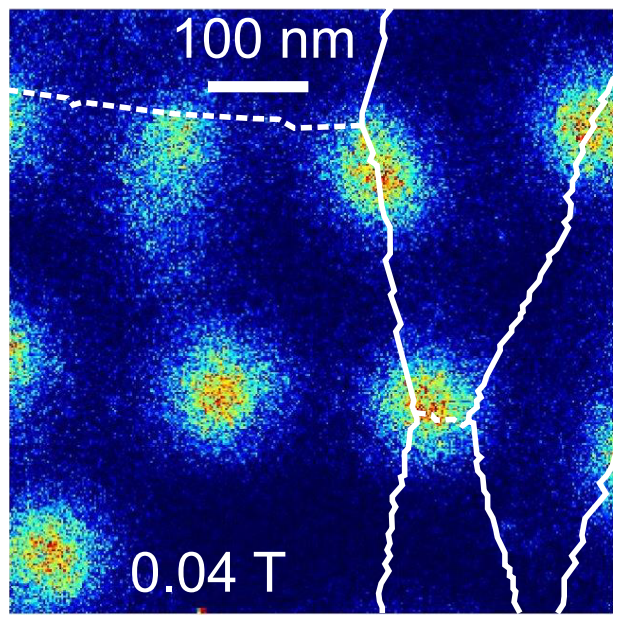

b
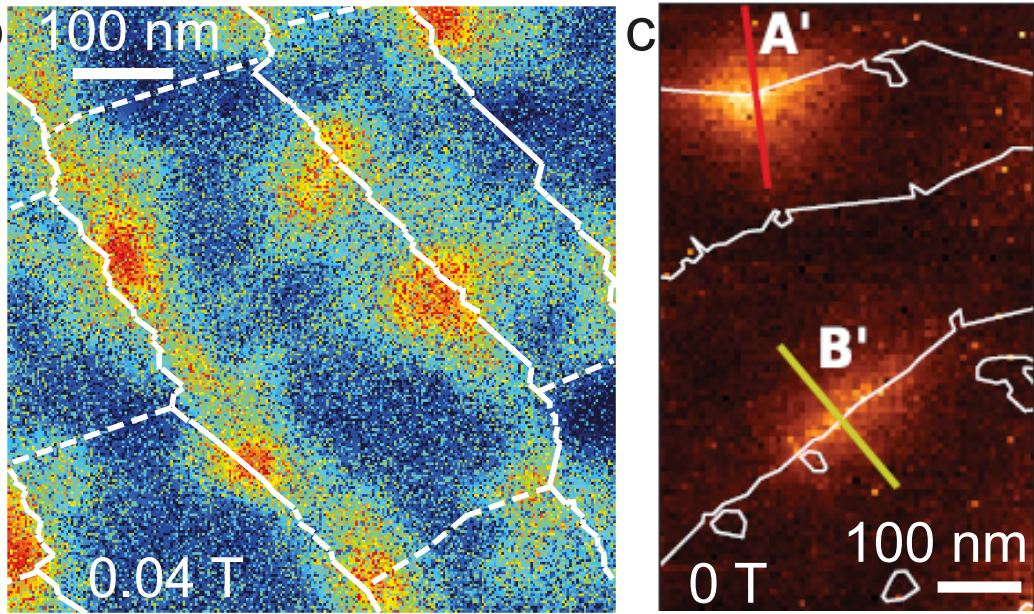
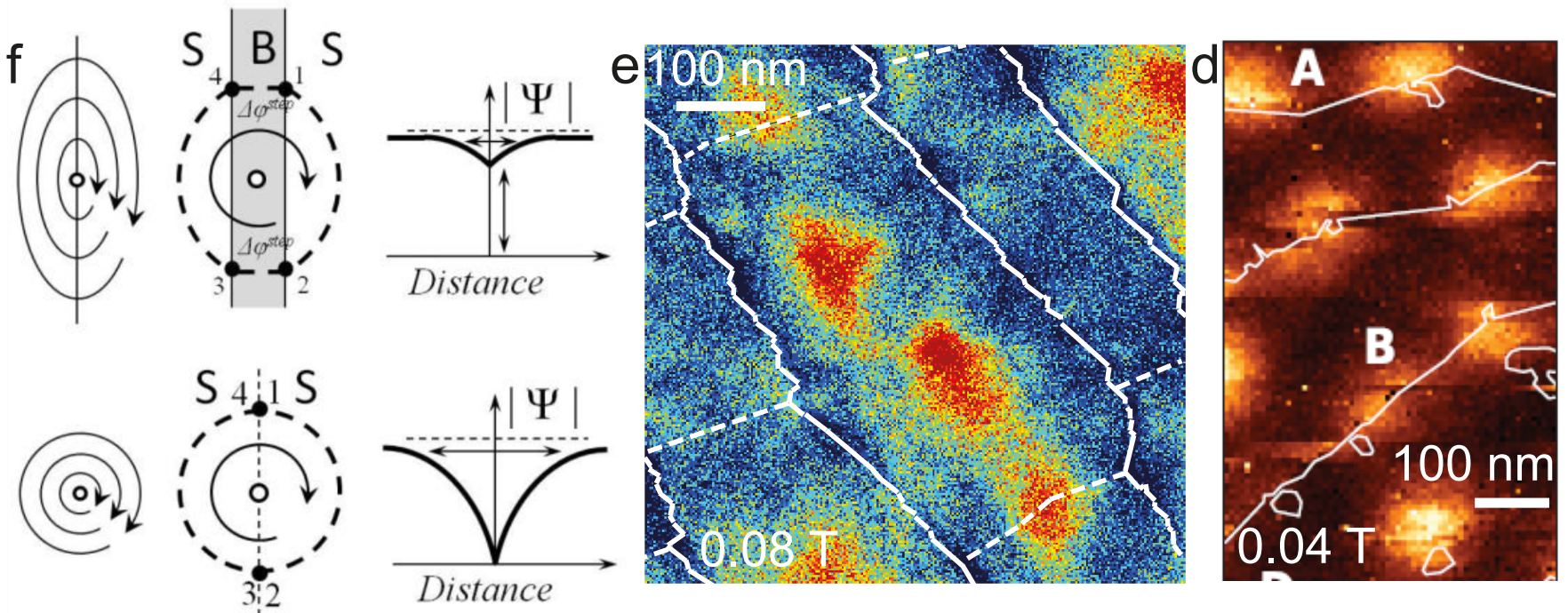

FIG. 21: Different superconducting behaviors of atomic step edges in $\mathbf{P b}$ and In monolayers deduced from vortex arrangements. Different vortex arrangements in SIC- $\mathrm{Pb}(\mathrm{a}), \sqrt{7} \times \sqrt{3}-\mathrm{Pb}(\mathrm{b}, \mathrm{e})$ and In monolayers (c,d). Panels a) to e) show zero-bias $d I / d V$ conductance maps. a): in SIC-Pb vortices have conventional round cores; monoatomic steps do not perturb their shapes significantly. b): in $\sqrt{7} \times \sqrt{3}$-Pb and c) in $\sqrt{7} \times \sqrt{3}$-In-monolayers at low field all vortices are pinned at step edges; the vortex cores are elongated along the steps direction. The spectral STS contrast is particularly low for some of these vortices. They are identified as mixed Abrikosov-Josephson vortices (see text). d), e): At higher field, when the steps get saturated with Abrikosov-Josephson vortices, new vortices of Abrikosov type (round) occupy the terraces (in d at 0.08T the differential map shows only the additional vortices that appeared when the field was increased from $0.04 \mathrm{~T}$ to $0.08 \mathrm{~T}$.) $\mathrm{f}$ ): Deformation of the vortex core located at a Josephson junction (top three schematics) as compared to a round Abrikosov vortex core in an homogeneous superconductor (bottom three schematics). Left panels: current flow; middle panels: evolution of the superconducting phase; right panels: spatial distribution of the superconducting order parameter. The phase singularity in the left and middle panels is represented by an open circle. After Brun et al. ${ }^{112}$ and Yoshizawa et al. ${ }^{113}$. 